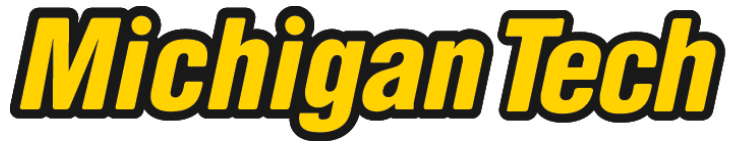 \\ Michigan Technological University Create the Future Digital Commons @ Michigan Tech
}

2013

\section{Application of MFBD Algorithms to Image Reconstruction Under Anisoplanatic Conditions}

Glen E. Archer

Michigan Technological University

Follow this and additional works at: https://digitalcommons.mtu.edu/etds

Part of the Electrical and Computer Engineering Commons

Copyright 2013 Glen E. Archer

\section{Recommended Citation}

Archer, Glen E., "Application of MFBD Algorithms to Image Reconstruction Under Anisoplanatic Conditions", Dissertation, Michigan Technological University, 2013.

https://doi.org/10.37099/mtu.dc.etds/780

Follow this and additional works at: https://digitalcommons.mtu.edu/etds

Part of the Electrical and Computer Engineering Commons 
APPLICATION OF MFBD ALGORITHMS TO IMAGE RECONSTRUCTION UNDER ANISOPLANATIC CONDITIONS

By

Glen E. Archer

\begin{abstract}
A DISSERTATION
Submitted in partial fulfillment of the requirements for the degree of DOCTOR OF PHILOSOPHY

In Electrical Engineering
\end{abstract}

MICHIGAN TECHNOLOGICAL UNIVERSITY

2013

(C) 2013 Glen E. Archer 
This dissertation has been approved in partial fulfillment of the requirements for the Degree of DOCTOR OF PHILOSOPHY in Electrical Engineering.

Department of Electrical and Computer Engineering

Dissertation Advisor: $\quad$ Dr. Michael C. Roggemann

Committee Member: $\quad$ Dr. Barbara S. Bertram

Committee Member: Dr. Warren F. Perger

Committee Member: Dr. Timothy J. Schulz

Department Chair: Dr. Daniel R. Fuhrmann 


\section{Dedication}

This document is dedicated to my wife, Ruth. Without her steadfast patience this work would not have been possible. Without her presence in my life it would have no meaning. 


\section{Contents}

List of Figures $\ldots \ldots \ldots \ldots \ldots \ldots \ldots \ldots \ldots$. . . . . . . . . . . . . . . . . .

List of Tables $\ldots \ldots \ldots \ldots \ldots \ldots \ldots \ldots \ldots \ldots \ldots \ldots \ldots \ldots$

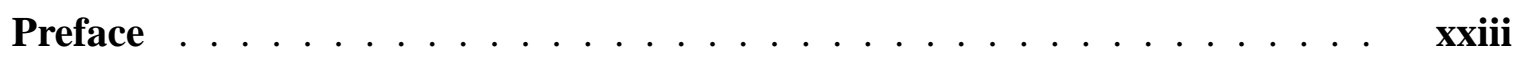

Acknowledgments $\ldots \ldots \ldots \ldots \ldots \ldots \ldots \ldots \ldots \ldots \ldots \ldots \ldots$

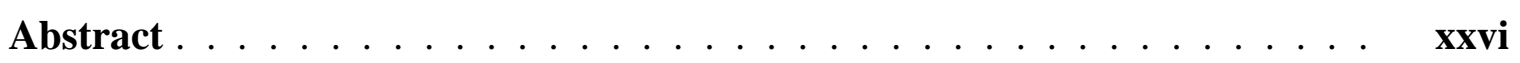

1 Introduction $\ldots \ldots \ldots \ldots \ldots \ldots \ldots \ldots \ldots \ldots \ldots \ldots$

1.1 Atmospheric Turbulence . . . . . . . . . . . . . . . . 5

1.1.1 Kolmogorov Turbulence . . . . . . . . . . . . . . . . . 5

1.1.2 Index of Refraction Structure Constant $C_{n}^{2} \ldots \ldots \ldots \ldots$

1.1.3 Anisoplanatism . . . . . . . . . . . . . . . . 9 9

1.1.4 Zernike Polynomials . . . . . . . . . . . . . . . 13

1.2 Method . . . . . . . . . . . . . . . . 15

1.3 Summary of Key Results . . . . . . . . . . . . . . . . . 17

1.4 Organization $\ldots \ldots \ldots \ldots \ldots \ldots \ldots \ldots \ldots \ldots \ldots \ldots$ 
2 Reconstruction of Long Horizontal-Path Images under Anisoplanatic conditions Using Multi-Frame Blind Deconvolution . . . . . . . . . . 19

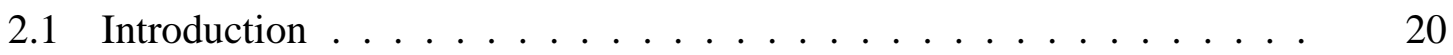

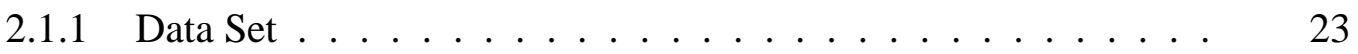

2.1.2 Gaussian Noise Model MFBD ... . . . . . . . . . . 26

2.1.3 Poisson noise model MFBD . . . . . . . . . . . . . 29

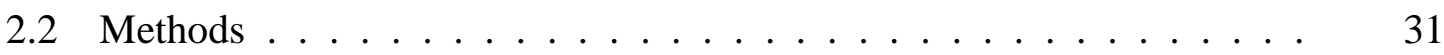

2.2.1 L-BFGS Optimization . . . . . . . . . . . . 33

2.2.2 Reconstruction Processing ............... 35

2.2.3 Number of Zernike Terms Needed in the Optimization Process . 36

2.3 Results . . . . . . . . . . . . . . . . . . 38

2.3.1 Gaussian Noise Model . . . . . . . . . . . . . 38

2.3.1.1 Case 1 Low Condition $C_{N}^{2}=2.25 \times 10^{-14} m^{(-2 / 3)} \quad . \quad 38$

2.3.1.2 Case 2 Moderate Condition $C_{N}^{2}=3.75 \times 10^{-14} m^{(-2 / 3)} \quad 40$

2.3.1.3 Case 3 Severe Condition $C_{N}^{2}=5.25 \times 10^{-14} m^{(-2 / 3)} \quad . \quad 43$

2.3.2 Poisson Noise Model Mean Photon Rate $2 \times 10^{6}, 30$ photons per pixel .............................. 46

2.3.2.1 Case 1 Low Condition $C_{N}^{2}=2.25 \times 10^{-14} m^{(-2 / 3)} \quad . \quad 47$

2.3.2.2 Case 2 Moderate Condition $C_{N}^{2}=3.75 \times 10^{-14} m^{(-2 / 3)} \quad 48$

2.3.2.3 Case 3 Severe Condition $C_{N}^{2}=5.25 \times 10^{-14} m^{(-2 / 3)} \quad . \quad 50$

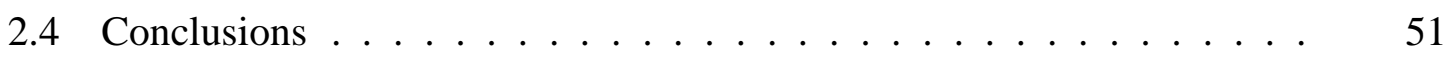


3 A Comparison of the MSE Performance of Speckle and MFBD Image Reconstruction Techniques $\ldots \ldots \ldots \ldots \ldots$

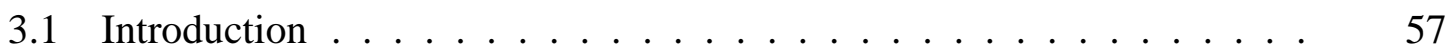

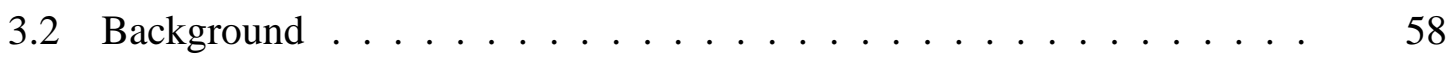

3.2.1 Horizontal Imaging . . . . . . . . . . . . . . 58

3.2.2 Speckle Imaging ........................ 62

3.2.3 MFBD: Gaussian Noise Model . . . . . . . . . . . . . . 65

3.2.4 MFBD: Poisson noise model . . . . . . . . . . . 70

3.3 Methods ............................ 71

3.4 Comparison of Bispectrum and MFBD methods under Gaussian and Poisson noise models . . . . . . . . . . . . . . 75

3.4.1 Gaussian Noise Model . . . . . . . . . . . . 75

3.4.1.1 Case 1 Low Condition $C_{N}^{2}=2.25 \times 10^{-14} m^{(-2 / 3)} \quad \ldots \quad 75$

3.4.1.2 Case 2 Moderate Condition $C_{N}^{2}=3.75 \times 10^{-14} m^{(-2 / 3)} \quad 76$

3.4.1.3 Case 3 Severe Condition $C_{N}^{2}=5.25 \times 10^{-14} m^{(-2 / 3)} . \quad 78$

3.4.2 Poisson Noise Model Mean Photon Rate $2 \times 10^{6}, 30$ photons per

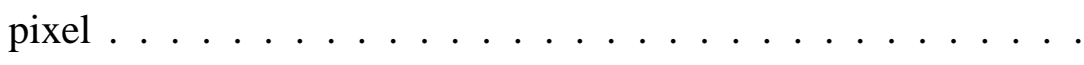

3.4.2.1 Case 1 Low Condition $C_{N}^{2}=2.25 \times 10^{-14} m^{(-2 / 3)} \quad$.

3.4.2.2 Case 2 Moderate Condition $C_{N}^{2}=3.75 \times 10^{-14} m^{(-2 / 3)}$

3.4.2.3 Case 3 Severe Condition $C_{N}^{2}=5.25 \times 10^{-14} m^{(-2 / 3)}$. 
3.4.3 Poisson Noise Model Mean Photon Rate $1 \times 10^{6}, 15$ photons per

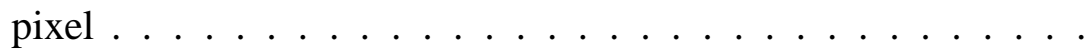

3.4.3.1 Case 1 Low Condition $C_{N}^{2}=2.25 \times 10^{-14} m^{(-2 / 3)} \quad \ldots$

3.4.3.2 Case 2 Moderate Condition $C_{N}^{2}=3.75 \times 10^{-14} m^{(-2 / 3)}$

3.4.3.3 Case 3 Severe Condition $C_{N}^{2}=5.25 \times 10^{-14} m^{(-2 / 3)}$.

3.4.4 Poisson Noise Model Mean Photon Rate $5 \times 10^{5}, 8$ photons per

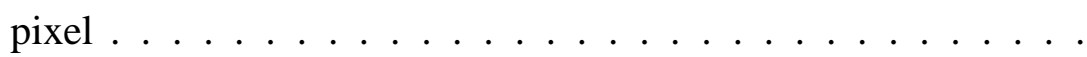

3.4.4.1 Case 1 Low Condition $C_{N}^{2}=2.25 \times 10^{-14} m^{(-2 / 3)} \quad$.

3.4.4.2 Case 2 Moderate Condition $C_{N}^{2}=3.75 \times 10^{-14} m^{(-2 / 3)}$

3.4.4.3 Case 3 Severe Condition $C_{N}^{2}=5.25 \times 10^{-14} m^{(-2 / 3)}$.

3.5 Conclusions . . . . . . . . . . . . . . . .

4 MFBD Estimator Performance With Field Data . . . . . . . . . . . . . .

4.1 Introduction . . . . . . . . . . . . . .

4.21400 EDT 1 July, 14 July 2009 Image Processing . . . . . . . . . . .

4.3 1900 EDT 24 July 2009 Image Processing

4.4 Conclusions

5 The Effect of Using Bispectrum as the Initial Estimate for the MFBD

Estimator

5.1 Introduction $\ldots \ldots \ldots \ldots$

5.2 Bispectrum initial estimate, limited iterations

5.3 B-MFBD run to convergence 
5.4 Conclusions .................................... 110

6 Conclusion and Thoughts on Future Work . . . . . . . . . . . . . 112

6.1 Conclusion .............................. 112

6.2 Future Work ...................... 115

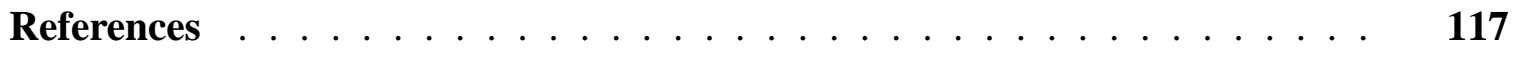

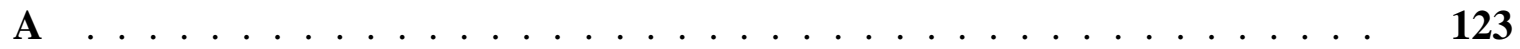

A.1 MFBD processing of the "Boats" Data Set . . . . . . . . . 123

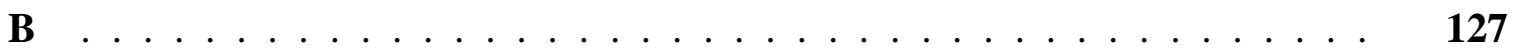

B.1 Letters of Permission . . . . . . . . . . . . . 127

\section{List of Figures}

1.1 Imaging Problem $\ldots \ldots \ldots \ldots$

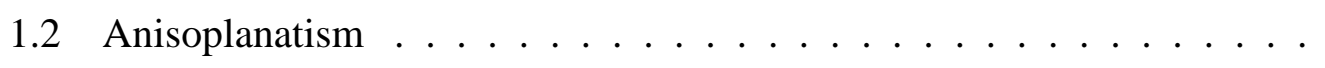


1.3 Anisoplanatic Pincushion Point Spread function. Figure (a) shows a pin cushion array of point sources corrupted under moderate turbulence condition. Figure (b) shows a 22 x 21 pixel extract, centered on the pixel location $(30,30)$. Figure (c) is a similar extract centered on location (230,

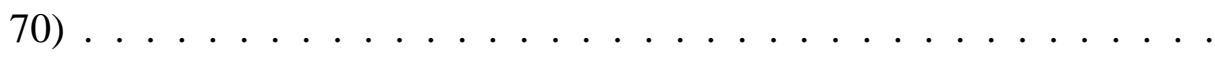

1.4 Cartesian coordinates mapped onto polar coordinates . . . . . . . .

2.1 Horizontal Imaging Simulator Output. Single image representative samples of the horizontal image simulator output (a) Diffraction-Limited Image, (b) Image for $C_{N}^{2}=2.25 \times 10^{-14} m^{(-2 / 3)}$, (c) Image for $C_{N}^{2}=3.25 \times$ $10^{-14} m^{(-2 / 3)},(\mathrm{d})$ Image for $C_{N}^{2}=5.25 \times 10^{-14} m^{(-2 / 3)} \ldots \ldots \ldots$

2.2 Padding Example . . . . . . . . . . . . . . . . .

2.3 Gaussian MSE vs Number of Function Calls . . . . . . . . . . . . .

2.4 MFBD Processing Time vs $N_{z}$ and $N_{f} \ldots \ldots \ldots \ldots$

2.5 Demonstration of the residual post-reconstruction MSE as the number of Zernike coefficients is varied. With the number of frames held constant at 50 the number of Zernike coefficients are varied from 10 to 100 for (a) Low condition $C_{N}^{2}=2.25 \times 10^{-14} m^{(-2 / 3)}$ (b) Moderate condition $C_{N}^{2}=3.75 \times$ $10^{-14} m^{(-2 / 3)}$ (c) Severe condition $C_{N}^{2}=5.25 \times 10^{-14} m^{(-2 / 3)} \ldots \ldots$

2.6 Mean Square Error vs. number of frames. Case 1 Low turbulence $C_{N}^{2}=$ $2.25 \times 10^{-14} m^{(-2 / 3)}$ with 30 and 60 Zernike terms $\ldots \ldots \ldots$ 
2.7 Residual reconstruction MSE as the number of frames used in the reconstruction is increased. The solid lines represent the mean value over $\mathrm{N}$ reconstructions, and the mean error over the entire data set. Dashed lines represent the variance of the estimate for a given number of frames used in the reconstruction. Case 1 Low turbulence $C_{N}^{2}=2.25 \times 10^{-14} m^{(-2 / 3)} N_{z}=$ 30

2.8 Residual reconstruction MSE as the number of frames used in the reconstruction is increased in the presence of additive noise. The solid lines represent the mean reconstruction error for $\sigma_{n}=1$ and $\sigma_{n}=10$. The dotted lines represent the mean performance for $\sigma_{n}=2,3 \ldots 9$. The dashed lines at the limit of the plots represents the variance for the two bounding cases. Case 1 Low turbulence $C_{N}^{2}=2.25 \times 10^{-14} m^{(-2 / 3)} N_{z}=30 \ldots \ldots$

2.9 Case 1 sample images. Compares the (a) diffraction-limited image with (b) the single sample image and (c) a sample reconstructed object. . . . . .

2.10 Case 2 sample images. Compares the (a) diffraction-limited image with (b) the single sample image and (c) a sample reconstructed object. . . . . .

2.11 Mean Square Error vs. number of frames. Case 2 Moderate turbulence $C_{N}^{2}$ $=3.75 \times 10^{-14} m^{(-2 / 3)}$ with 30 and 60 Zernike terms $\ldots \ldots \ldots$ 
2.12 Residual reconstruction MSE as the number of frames used in the reconstruction is increased. The solid lines represent the mean value over $\mathrm{N}$ reconstructions, and the mean error over the entire data set. Dashed lines represent the variance of the estimate for a given number of frames used in the reconstruction. Case 2 Moderate turbulence $C_{N}^{2}=3.75 \times 10^{-14} m^{(-2 / 3)}$

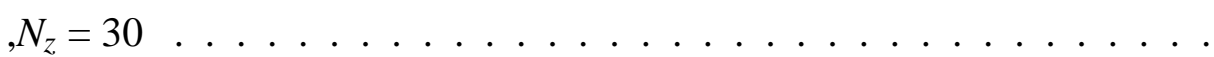

2.13 Residual reconstruction MSE as the number of frames used in the reconstruction is increased in the presence of additive noise. The solid lines represent the mean reconstruction error for $\sigma_{n}=1$ and $\sigma_{n}=10$. The dotted lines represent the mean performance for $\sigma_{n}=2,3 \ldots 9$. The dashed lines at the limit of the plots represents the variance for the two bounding cases. Case 2 Moderate turbulence $C_{N}^{2}=3.75 \times 10^{-14} m^{(-2 / 3)} N_{z}=30$.

2.14 Case 3 sample images. Compares the (a) diffraction-limited image with (b) the single sample image and (c) a sample reconstructed object. . . . . .

2.15 Mean Square Error vs. number of frames. Case 3 Severe turbulence $C_{N}^{2}=$ $5.25 \times 10^{-14} m^{(-2 / 3)}$ with 30 and 60 Zernike terms $\ldots \ldots \ldots$ 
2.16 Residual reconstruction MSE as the number of frames used in the reconstruction is increased. The solid lines represent the mean value over $\mathrm{N}$ reconstructions, and the mean error over the entire data set. Dashed lines represent the variance of the estimate for a given number of frames used in the reconstruction. Case 3 Severe turbulence $C_{N}^{2}=5.25 \times 10^{-14} m^{(-2 / 3)} N_{z}$

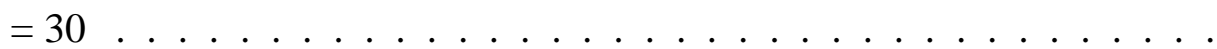

2.17 Residual reconstruction MSE as the number of frames used in the reconstruction is increased in the presence of additive noise. The solid lines represent the mean reconstruction error for $\sigma_{n}=1$ and $\sigma_{n}=10$. The dotted lines represent the mean performance for $\sigma_{n}=2,3 \ldots 9$. The dashed lines at the limit of the plots represents the variance for the two bounding cases. Case 3 Severe turbulence $C_{N}^{2}=5.25 \times 10^{-14} m^{(-2 / 3)}$ with $N_{z}=30$

2.18 Case 1 sample images, Mean Photon Rate $2 \times 10^{6}, C_{N}^{2}=2.25 \times$ $10^{-14} m^{(-2 / 3)}$. Compares the (a) diffraction-limited image with (b) a single sample image and (c) a sample reconstructed object. . . . . . . . . . . .

2.19 Mean Square Error vs. number of frames. Poisson Case 1 Low turbulence $C_{N}^{2}=2.25 \times 10^{-14} m^{(-2 / 3)}$, mean photon rate $2 \times 10^{6} \ldots \ldots . .$.

2.20 Case 2 sample images, Mean Photon Rate $2 \times 10^{6}, C_{N}^{2}=3.75 \times$ $10^{-14} m^{(-2 / 3)}$. Compares the (a) diffraction-limited image with (b) a single sample image and (c) a sample reconstructed object. . . . . . . . . . . . 
2.21 Mean Square Error vs. number of frames. Poisson Case 2 Moderate turbulence $C_{N}^{2}=3.75 \times 10^{-14} m^{(-2 / 3)}$, mean photon rate $2 \times 10^{6} \ldots$.

2.22 Case 3 sample images, Mean Photon Rate $2 \times 10^{6}, C_{N}^{2}=5.25 \times$ $10^{-14} m^{(-2 / 3)}$. Compares the (a) diffraction-limited image with (b) a single sample image and (c) a sample reconstructed object. . . . . . . . . . .

2.23 Mean Square Error vs. number of frames. Poisson Case 3 Severe turbulence $C_{N}^{2}=2.25 \times 10^{-14} m^{(-2 / 3)}$, mean photon rate $2 \times 10^{6} \ldots$.

3.1 Horizontal Imaging Simulator Output. Single image representative samples of the horizontal image simulator output (a) Diffraction-Limited Image, (b) Image for $C_{N}^{2}=2.25 \times 10^{-14} m^{(-2 / 3)}$, (c) Image for $3.75 \times 10^{-14} m^{(-2 / 3)}$, (d) Image for $5.25 \times 10^{-14} m^{(-2 / 3)} \ldots \ldots \ldots \ldots \ldots$

3.2 Comparison of Bispectrum and MFBD Mean Square Error as the number of input image frames is increased. For MFBD, $N_{z}=30$, and the number of iterations was fixed at 25. For Bispectrum, $N_{p}=5 \alpha=.3$. Case 1 Low turbulence $C_{N}^{2}=2.25 \times 10^{-14} m^{(-2 / 3)} \ldots \ldots \ldots \ldots$

3.3 Case 1 sample images. Compares (a) a sample turbulent image (b) a sample MFBD reconstructed object and (c) a sample bispectrum reconstructed

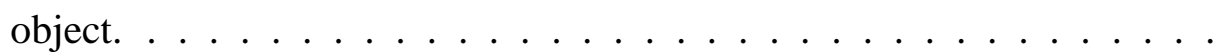

3.4 Case 2 sample images. Compares (a) a sample turbulent image (b) a sample MFBD reconstructed object and (c) a sample bispectrum reconstructed

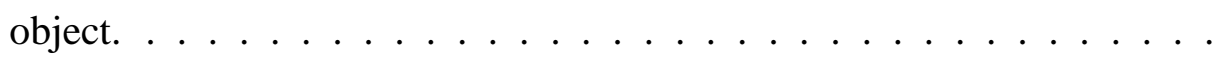


3.5 Comparison of Bispectrum and MFBD Mean Square Error as the number of input image frames is increased. For MFBD, $N_{z}=30$, and the number of iterations was fixed at 25. For Bispectrum, $N_{p}=5 \alpha=.3$. Case 2 Moderate turbulence $C_{N}^{2}=3.75 \times 10^{-14} m^{(-2 / 3)}$

3.6 Case 3 sample images. Compares (a) a sample turbulent image (b) a sample MFBD reconstructed object and (c) a sample bispectrum reconstructed

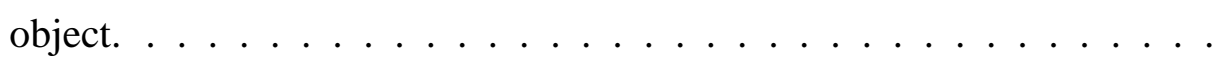

3.7 Comparison of Bispectrum and MFBD Mean Square Error as the number of input image frames is increased. For MFBD, $N_{z}=30$, and the number of iterations was fixed at 25. For Bispectrum, $N_{p}=5 \alpha=.3$. Case 3 Severe turbulence $C_{N}^{2}=5.25 \times 10^{-14} m^{(-2 / 3)} \ldots \ldots \ldots \ldots$

3.8 Comparison of Bispectrum and MFBD Mean Square Error as the number of input image frames is increased. For MFBD, $N_{z}=30$, and the number of iterations was fixed at 25. For Bispectrum, $N_{p}=5 \alpha=.3$. Poisson Case 1 Low condition $C_{N}^{2}=2.25 \times 10^{-14} m^{(-2 / 3)}$, mean photon rate $2 \times 10^{6}, 30$ photons per pixel $\ldots \ldots \ldots \ldots \ldots$

3.9 Case 1 Low condition sample images, Mean Photon Rate $2 \times 10^{6}, 30$ photons per pixel, $C_{N}^{2}=2.25 \times 10^{-14} m^{(-2 / 3)}$. Compares (a) a sample turbulent image (b) a sample MFBD reconstructed object $\left(N_{f}=175\right)$ and (c) a sample bispectrum $\left(N_{f}=200\right)$ reconstructed object. image. . . . . . 
3.10 Case 2 Moderate condition sample images, Mean Photon Rate $2 \times 10^{6}$, 30 photons per pixel, $C_{N}^{2}=3.75 \times 10^{-14} m^{(-2 / 3)}$. Compares (a) a sample turbulent image (b) a sample MFBD reconstructed $\left(N_{f}=175\right)$ object and (c) a sample bispectrum $\left(N_{f}=200\right)$ reconstructed object. . . . . . .

3.11 Comparison of Bispectrum and MFBD Mean Square Error as the number of input image frames is increased. For MFBD, $N_{z}=30$, and the number of iterations was fixed at 25. For Bispectrum, $N_{p}=5 \alpha=.3$. Poisson Case 2 Moderate condition $C_{N}^{2}=3.75 \times 10^{-14} \mathrm{~m}^{(-2 / 3)}$, mean photon rate $2 \times 10^{6}$ 30 photons per pixel $\ldots \ldots \ldots \ldots \ldots \ldots$

3.12 Comparison of Bispectrum and MFBD Mean Square Error as the number of input image frames is increased. For MFBD, $N_{z}=30$, and the number of iterations was fixed at 25. For Bispectrum, $N_{p}=5 \alpha=.3$. Poisson Case 3 Severe condition $C_{N}^{2}=2.25 \times 10^{-14} m^{(-2 / 3)}$, mean photon rate $2 \times 10^{6}, 30$ photons per pixel $\ldots \ldots \ldots \ldots \ldots \ldots \ldots$

3.13 Case 3 Severe condition sample images, Mean Photon Rate $2 \times 10^{6}, C_{N}^{2}=$ $5.25 \times 10^{-14} m^{(-2 / 3)}$. Compares (a) a sample simulated turbulent image (b) a sample MFBD reconstructed object, $N_{f}=175$, and (c) a sample bispectrum reconstructed object $N_{f}=200 \ldots \ldots \ldots \ldots$ 
3.14 Case 1 Low condition $C_{N}^{2}=2.25 \times 10^{-14} m^{(-2 / 3)}$, mean photon rate $1 \times$ $10^{6}$. Compares (a) a sample simulated turbulent image (b) a sample MFBD reconstricted object, $N_{f}=175$, and (c) a sample bispectrum reconstructed object $N_{f}=200 \ldots \ldots \ldots \ldots \ldots \ldots \ldots \ldots$

3.15 Comparison of Bispectrum and MFBD Mean Square Error as the number of input image frames is increased. For MFBD, $N_{z}=30$, and the number of iterations was fixed at 25. For Bispectrum, $N_{p}=5 \alpha=.3$. Poisson Case 1 Low turbulence $C_{N}^{2}=2.25 \times 10^{-14}$, mean photon rate $1 \times 10^{6} 15$ photons

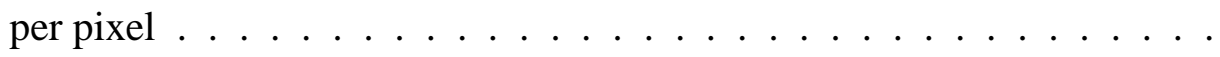

3.16 Comparison of Bispectrum and MFBD residual Mean Square Error as the number of input image frames is increased. For MFBD, $N_{z}=30$, and the number of iterations was fixed at 25. For Bispectrum, $N_{p}=5 \alpha=$ .3. Poisson Case 2 Moderate turbulence $C_{N}^{2}=3.75 \times 10^{-14} m^{(-2 / 3)}$, mean photon rate $1 \times 10^{6} 15$ photons per pixel . . . . . . . . . .

3.17 Case 2 Moderate turbulence $C_{N}^{2}=3.75 \times 10^{-14} m^{(-2 / 3)}$, mean photon rate $1 \times 10^{6}$.Compares (a) a sample turbulent image (b) a sample MFBD reconstructed object and (c) a sample bispectrum reconstructed object. .

3.18 Bispectrum MFBD Mean Square Error vs. number of frames. Poisson Case 3 Severe turbulence $C_{N}^{2}=2.25 \times 10^{-14} m^{(-2 / 3)}$, mean photon rate $1 \times 10^{6}$ 15 photons per pixel . . . . . . . . . . . . . 
3.19 Case 3 Severe turbulence $C_{N}^{2}=5.25 \times 10^{-14} m^{(-2 / 3)}$, mean photon rate $1 \times 10^{6}$.Compares (a) a sample turbulent image (b) a sample MFBD reconstructed object and (c) a sample bispectrum reconstructed object. .

3.20 Case 1 Low turbulencs $C_{N}^{2}=2.25 \times 10^{-14} m^{(-2 / 3)}$, mean photon rate $5 \times 10^{5}$. Compares (a) a sample turbulent image (b) a sample MFBD reconstructed object $N_{f}=175$, and (c) a sample $N_{f}=200$, reconstructed

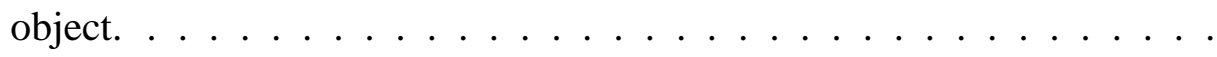

3.21 Comparison of Bispectrum and MFBD Mean Square Error as the number of input image frames is increased. For MFBD, $N_{z}=30$, and the number of iterations was fixed at 25. For Bispectrum, $N_{p}=5 \alpha=.3$. Poisson Case 1 Low turbulence $C_{N}^{2}=2.25 \times 10^{-14} m^{(-2 / 3)}$, mean photon rate $5 \times 10^{5}, 8$

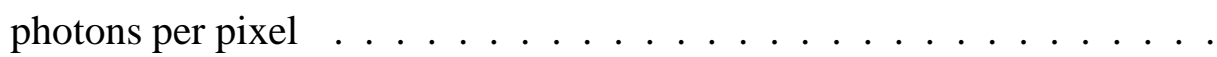

3.22 Case 2 Moderate turbulence $C_{N}^{2}=3.75 \times 10^{-14} m^{(-2 / 3)}$, mean photon rate $5 \times 10^{5}$. Compares (a) a sample turbulent image (b) a sample MFBD reconstructed object and (c) a sample bispectrum reconstructed object. .

3.23 Comparison of Bispectrum and MFBD Mean Square Error as the number of input image frames is increased. For MFBD, $N_{z}=30$, and the number of iterations was fixed at 25. For Bispectrum, $N_{p}=5 \alpha=.3$. Poisson Case 2 Moderate turbulence $C_{N}^{2}=3.75 \times 10^{-14} m^{(-2 / 3)}$, mean photon rate $5 \times 10^{5}$ 8 photons per pixel $\ldots \ldots \ldots \ldots$ 
3.24 Case 3 Severe turbulence $C_{N}^{2}=5.25 \times 10^{-14} m^{(-2 / 3)}$, mean photon rate $5 \times 10^{5}$.Compares (a) a sample turbulent image (b) a sample MFBD reconstructed object and (c) a sample bispectrum reconstructed object. .

3.25 Bispectrum and MFBD Mean Square Error vs. number of frames. Poisson Case 3 Severe turbulence $C_{N}^{2}=5.25 \times 10^{-14}$, mean photon rate $5 \times 10^{5}$

4.1 Field Data Reconstruction from 1 July 1400 EDT data set, using the Gaussian noise model MFBD reconstructor, $N_{f}=15, N_{\text {iter }}=20, r_{0} 4.6$ - $5.2 \mathrm{~cm}$. A sample input image is shown in Figure (a) and a sample reconstructed image in Figure (b)

4.2 Field Data Reconstruction from 14 July 1400 EDT data set, using the Gaussian noise model MFBD reconstructor, $N_{f}=15, N_{\text {iter }}=20, r_{0}$ 2.3-2.4 cm. A sample input image is shown in Figure (a) and a sample reconstructed image in Figure (b)

4.3 Field Data Reconstruction from 24 July 1900 data set, using the Gaussian noise model MFBD reconstructor, $N_{z}=35, N_{f}=25, N_{\text {iter }}=20, r_{0} 1.78$ - $2.8 \mathrm{~cm}$. A sample input image is shown in Figure (a) and a sample

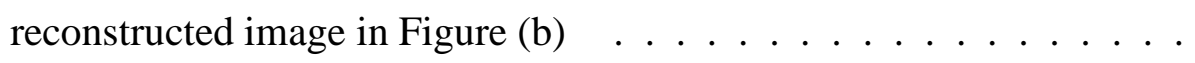

5.1 Mean Square Error performance vs. number of frames using the (a) MFBD alone, (b) Bispectrum alone, and (c) B-MFBD method using bispectrum to provide an initial estimate for processing with the MFBD estimator. Gaussian Case 1 low turbulence $C_{N}^{2}=2.25 \times 10^{-14} m^{(-2 / 3)}, N_{f}=15$. . 
5.2 Limited iteration B-MFBD Samples, Gaussian Case 1 low turbulence $C_{N}^{2}$ $=2.25 \times 10^{-14} m^{(-2 / 3)}$ images: (a) input frame, and (b) sample B-MFBD reconstructed object. . . . . . . . . . . . . . . 108

5.3 Mean Square Error performance vs. number of frames using the (a) MFBD, (b) Bispectrum, and (c) B-MFBD method using bispectrum to provide an initial estimate for processing with the MFBD estimator. Gaussian Case 2 moderate turbulence $C_{N}^{2}=3.75 \times 10^{-14} m^{(-2 / 3)}, N_{f}=15 \ldots \ldots$

5.4 Limited iteration B-MFBD Samples, Gaussian Case 2 moderate turbulence $C_{N}^{2}=3.75 \times 10^{-14} m^{(-2 / 3)}:$ (a) input frame, and (b) sample B-MFBD reconstructed object.

5.5 Mean Square Error performance vs. number of frames using the (a) MFBD, (b) Bispectrum, and (c) B-MFBD method using bispectrum to provide an initial estimate for processing with the MFBD estimator. Gaussian Case 3 severe turbulence $C_{N}^{2}=5.25 \times 10^{-14} m^{(-2 / 3)}, N_{f}=15 . \ldots \ldots$

5.6 Limited iteration B-MFBD Samples, Gaussian Case 3 severe turbulence $C_{N}^{2}=5.25 \times 10^{-14} m^{(-2 / 3)}$ images: (a) input frame, (b) sample B-MFBD reconstructed object . . . . . . . . . . . . .

5.7 B-MFBD run to convergence using SI initial estimate. (a) Low turbulence condition input image sample, (b) Initial estimate from SI as MFBD starting point, (c) B-MFBD output when run to convergence . . . . . . . . 
5.8 B-MFBD run to convergence using SI initial estimate. (a) Moderate turbulence condition input image sample, (b) Initial estimate from SI as MFBD starting point, (c) B-MFBD output when run to convergence . .

5.9 B-MFBD run to convergence using SI initial estimate. (a) Severe turbulence condition input image sample, (b) Initial estimate from SI as MFBD starting point, (c) B-MFBD output when run to convergence . .

A.1 Evaluation of Mean Square Error vs. number of frames used in the MFBD processing with $N_{z}=30$, and the number of iterations limited to 30 . (a) Gaussian Case 1 low turbulence $C_{N}^{2}=2.25 \times 10^{-14} m^{(-2 / 3)}$, (b) Gaussian Case 2 moderate turbulence $C_{N}^{2}=3.75 \times 10^{-14} m^{(-2 / 3)}$, (c) Gaussian Case 3 severe turbulence $C_{N}^{2}=5.25 \times 10^{-14} m^{(-2 / 3)} \ldots \ldots \ldots \ldots$

A.2 Diffraction limited image from the "Boats" data sets. . . . . . . . . .

A.3 Sample input and reconstructed image from the "Boats" data set processed with the MFBD algorithm, with $N_{z}=30$, and the number of iterations limited to 30. Gaussian Case 1 low turbulence $C_{N}^{2}=2.25 \times 10^{-14} m^{(-2 / 3)}$.

A.4 Sample input and reconstructed image from the "Boats" data set processed with the MFBD algorithm, with $N_{z}=30$, and the number of iterations limited to 30. Gaussian Case 2 moderate turbulence $C_{N}^{2}=3.75 \times$

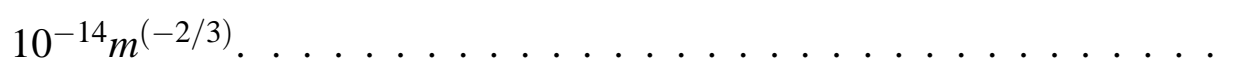


A.5 Sample input and reconstructed image from the "Boats" data set processed with the MFBD algorithm, with $N_{z}=30$, and the number of iterations limited to 30 , Gaussian Case 3 severe turbulence $C_{N}^{2}=5.25 \times$ $10^{-14} m^{(-2 / 3)} \ldots \ldots \ldots \ldots \ldots \ldots \ldots \ldots \ldots \ldots$

\section{List of Tables}

2.1 Atmospheric Simulation Turbulence Conditions . . . . . . . . . .

2.2 Selection of image padding . . . . . . . . . . . . .

2.3 Input Object and Zernike Coefficients. The initial guess at the object estimate is stripped into a vector with the intensities at the beginning and the initial guess at each input image's Zernike coefficients follow. . . . .

2.4 Vectorized Reconstructed Object and Zernike Coefficients. The estimate is returned as a vector with the estimated object pixel intensities at the beginning and the estimate of each input image's Zernike coeffiecients at

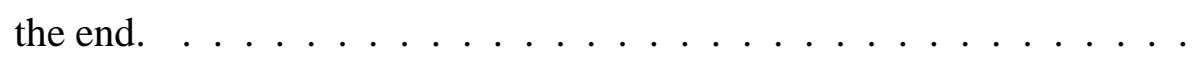


4.1 Date, time, and wave front sensor turbulence measurement range taken at approximately the same time as the images in data set 1, 2, and 3 were

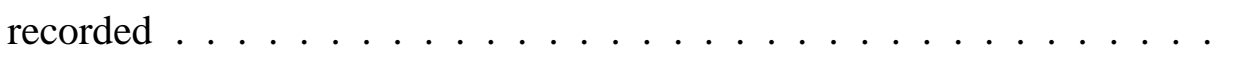

5.1 Mean and variance for MFBD, Bispectrum and the hybrid B-MFBD methods for Low turbulence $C_{N}^{2}=2.25 \times 10^{-14} m^{(-2 / 3)}$, Moderate Condition $C_{N}^{2}=3.75 \times 10^{-14} m^{(-2 / 3)}$, and Severe Condition $C_{N}^{2}=5.25 \times$ $10^{-14} m^{(-2 / 3)}$. Processing was accomplished with $N_{f}=15, N_{z}=15$ and the exit criteria for the optimization routine set at 40 iterations. . . . . . . . 


\section{Preface}

This dissertation is composed of two journal articles written by myself, Dr. Jeremy Bos, and my advisor, Dr. Michael Roggemann. The material in Chapter 2 was first published on-line August 232013 Optical Engineering Journal as "Reconstruction of long horizontal-path images under anisoplanatic conditions using multiframe blind deconvolution." Dr. Bos and Dr. Roggemann are listed as contributing authors. Dr Roggemann provided the initial idea for the paper and helped me develop the paper. Dr Bos provided the simulator that produced the data for analysis and edited the content. As the first author, I accomplished all other contributing work, wrote, and prepared the manuscript and supporting analysis using the MFBD reconstructor. The L-BFGS optimizer code was created by Joel LeBlanc of the Michigan Tech Research Institute. Dr Roggemann also provided other MATLAB code which I modified to adapt it to my purposes. I wrote other code to conduct the parametric analyses for the papers. The material in Chapters 3, 4, and 5 has been accepted for publication by Optical Engineering under a working title, "Comparison of Bispectrum, MFBD, and Hybrid Bispectrum-MFBD Image Reconstruction Techniques for Anisoplanatic, Long Horizontal-Path Imaging." The simulated data used in this work was produced using the simulator developed by Dr Bos. I used the bispectrum reconstructor he developed to compare its performance to the MFBD estimator and to provide the initial stage of the hybrid Bispectrum-MFBD reconstructor. The field data was provided by Dr

Roggemann. The work presented in this dissertation are the results of my own effort 
strengthened by feedback and recommendations from Dr Roggemann and Dr Bos. 


\section{Acknowledgments}

I would like to thank my adviser, Dr Mike Roggemann, and my committee for their efforts to guide me to a successful conclusion; my friend Jeremy Bos who was always helpful when I needed encouragement, Gowtham who helped me with LATEX and Larry Millward, my high school physics teacher, who with out knowing it, set me on this path in 1970 . 


\section{Abstract}

All optical systems that operate in or through the atmosphere suffer from turbulence induced image blur. Both military and civilian surveillance, gun-sighting, and target identification systems are interested in terrestrial imaging over very long horizontal paths, but atmospheric turbulence can blur the resulting images beyond usefulness. My dissertation explores the performance of a multi-frame-blind-deconvolution technique applied under anisoplanatic conditions for both Gaussian and Poisson noise model assumptions. The technique is evaluated for use in reconstructing images of scenes corrupted by turbulence in long horizontal-path imaging scenarios and compared to other speckle imaging techniques. Performance is evaluated via the reconstruction of a common object from three sets of simulated turbulence degraded imagery representing low, moderate and severe turbulence conditions. Each set consisted of 1000 simulated, turbulence degraded images. The MSE performance of the estimator is evaluated as a function of the number of images, and the number of Zernike polynomial terms used to characterize the point spread function.

I will compare the mean-square-error (MSE) performance of speckle imaging methods and a maximum-likelihood, multi-frame blind deconvolution (MFBD) method applied to long-path horizontal imaging scenarios. Both methods are used to reconstruct a scene from simulated imagery featuring anisoplanatic turbulence induced aberrations. 
This comparison is performed over three sets of 1000 simulated images each for low, moderate and severe turbulence-induced image degradation. The comparison shows that speckle-imaging techniques reduce the MSE 46 percent, 42 percent and 47 percent on average for low, moderate, and severe cases, respectively using 15 input frames under daytime conditions and moderate frame rates. Similarly, the MFBD method provides, 40 percent, 29 percent, and 36 percent improvements in MSE on average under the same conditions. The comparison is repeated under low light conditions (less than 100 photons per pixel) where improvements of 39 percent, 29 percent and 27 percent are available using speckle imaging methods and 25 input frames and 38 percent, 34 percent and 33 percent respectively for the MFBD method and 150 input frames. The MFBD estimator is applied to three sets of field data and the results presented. Finally, a combined Bispectrum-MFBD Hybrid estimator is proposed and investigated. This technique consistently provides a lower MSE and smaller variance in the estimate under all three simulated turbulence conditions. 


\section{Chapter 1}

\section{Introduction}

Every optical system using light that has propagated any appreciable distance through the atmosphere will suffer, to some degree, from turbulence induced phase aberrations. In addition to phase errors at the aperture, light propagating over longer distances, or through stronger turbulence, will cause images to suffer from anisoplanatic, and possibly scintillation effects as well. Often the image blur induced by these phase aberrations is the limiting factor in the ability to recognize details of objects in the scene. Unless the seeing conditions are very favorable, anisoplanatism will dominate most practical horizontal imaging situations. The resulting distortion will limit the performance of any optical system operating in such a turbulent atmosphere, frequently causing the observed scene to be blurred beyond usefulness[1]. 
Many strategies have been proposed to estimate the true scene from turbulence corrupted images. All of them represent a compromise between how quickly the technique converges to a solution, the accuracy, and robustness of the technique. Quick and accurate convergence under a wide range of atmospheric seeing conditions, regardless of the scene illumination, while remaining transportable by a single operator are desirable characteristics. Commercial and military systems must allow relatively unsophisticated users to operate the system successfully. Power and portability constraints may eliminate image processing systems that require additional hardware beyond the basic imaging system. In addition, remotely piloted surveillance systems must transmit images to remote users over wireless channels that have limited bandwidth. As a result, currently fielded surveillance systems compress images prior to transmission [2]. An image processing system that could reconstruct images corrupted by turbulence prior to compression and transmission would allow such systems to make more efficient use of available bandwidth. The potential benefits of real-time, or near-real-time, turbulent image processing hardware for long-range surveillance and weapons targeting are sufficient to motivate significant commitment of both time and money to their development. However, quantitative comparisons between potential candidates are necessary to confidently decide on a preferred processing algorithm. Thus, effort must be directed toward the examination and selection of computationally efficient and robust image reconstruction techniques.

A variety of techniques including speckle imaging, adaptive optics, and iterative multi-frame blind deconvolution have been devised to correct the turbulence induced 
phase aberrations in vertical imaging applications. Labeyrie originally proposed the use of short-time exposure images to recover the intensity of an object's Fourier transform [3] while acknowledging that without the object's phase spectrum, an image of the object could not be reconstructed. Ayers and Dainty [4], among others, [5] determined that near diffraction-limited phase information could be recovered from multiple speckle-masked images through the use of Knox-Thompson or triple correlation and bispectrum techniques [6]. The recovered phase and intensity information are used to reconstruct nearly diffraction-limited images of astronomical objects without the need for a point source within the isoplanatic field.

Adaptive optic strategies using wave front sensors to control deformable mirrors have been used in celestial observation systems for many years [7]. One limitation to adaptive optic systems is that they only correct well over a few multiples of the isoplanatic angle, $\theta_{0}$, which will be defined later in Section 1.1. Common horizontal surveillance scenarios observe extended scenes that cover many times $\theta_{0}$. Techniques that apply a known phase term to the captured image, $[8,9,10]$ have also been used with some success. Paxman and Schulz explored this problem by creating phase diversity across multiple speckle images. This technique uses two simultaneous measurements - an in-focus image and another with a known degree of defocus applied before the second measurement is taken[11]. This technique is limited to fields of view that do not appreciably exceed the isoplanatic angle existing at the moment the image was captured, require substantial hardware, and divide signal energy between two detectors. 
Post-detection processing of wide field of view images captured with short-exposure times is another alternative. Fraser et al, described a technique for point-by-point registration of anisoplanatic speckle images to reduce motion blur and prepare the images for other deconvolution strategies[12]. The use of these strategies in surveillance imaging is largely unexplored. Blind deconvolution techniques have also been applied to reconstructing turbulence corrupted images. Ayers and Dainty pioneered the application of an iterative blind deconvolution technique to a single image degraded by atmospheric turbulence [4]. Schulz extended that method to include multiple input images and developed a penalized-maximum-likelihood algorithm to avoid the trivial solution that incorrectly concludes that the optical system's PSF is a Dirac delta function and the most likely object estimate is the observed turbulent image[13]. Little work has been done to compare the performance characteristics of the MFBD and speckle imaging techniques under anisoplanatic conditions.

Hybrid hardware-software strategies offer the potential to produce on-the-fly estimates of scenes but require substantial investment in both hardware and software to produce results [14]. Carrano proposed the use of speckle imaging for horizontal imaging [15] and EM Photonics has developed an embedded system using the technique to reconstruct turbulent images over horizontal paths in near real time[16]. Both Carrano and Bos ignore anisoplanatism in their processing but nevertheless produce reconstructions with reduced error[1]. Bos and Roggemann [1] have reported the use of software reconstruction techniques using the bi-spectrum method in nearly real-time. 


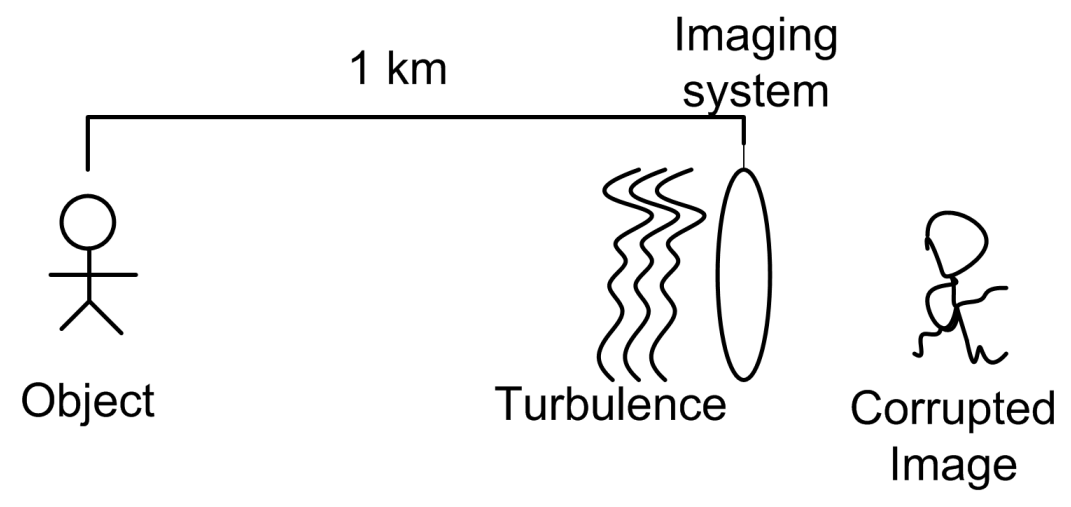

Figure 1.1: Imaging Problem

\subsection{Atmospheric Turbulence}

I begin the discussion by examining some assumptions regarding the horizontal imaging problem. Since the days of Newton, we have known that the resolution available to an imaging system is limited by atmospheric turbulence. When the sun shines on the earth, energy is transferred into the soil and the atmosphere, heating it. The resulting convection results in volumes of warm air rising and cooler air falling, creating vortices and eddies in the atmosphere. We experience this random turbulent fluid flow as wind.

\subsubsection{Kolmogorov Turbulence}

Kolmogorov [17] proposed a model for the random turbulent flow in the atmosphere. He described the transfer of energy from the sun to large scale motions, $L_{0}$, that can be 
continental in extent and have very large Reynolds numbers. The Reynolds number is a dimensionless quantity that relates inertial and viscous energy in fluids.

$$
R e=\frac{l v_{\text {avg }}}{k_{v}}
$$

where $v_{\text {avg }}$ is the average velocity of the volume, and $k_{v}$ is the kinematic viscosity of the atmosphere and $l$ is the scale size. Inertial energy is transferred to smaller and smaller scale motions, $l_{0}$, which can be as small as a few centimeters, with increasingly small Reynolds numbers. When the Reynolds number is large, atmospheric motion is dominated by inertial forces; and is turbulent. When the Reynolds number is low, atmospheric motion is dominated by viscous forces and the motion is smooth. The kinematic viscosity of the atmosphere is $k_{v}=1.5 \times 10^{-5} \mathrm{~m}^{2} / \mathrm{s}$. For scale sizes of 1 meter and average wind speed of 1 $\mathrm{m} / \mathrm{s}$ the resulting Reynolds number is $R_{e}=6.7 \times 10^{5}$. This is large enough to ensure that the atmosphere is nearly always turbulent. As the scale of the atmospheric volume decreases, the Reynolds number falls below a critical value and the energy in the volume is dissipated in the viscosity of the atmosphere where it appears as heat and the atmosphere is no longer turbulent[17]. Kolmogorov refers to the scale sizes between the large scale and small scale as the inertial sub range. It was assumed that in these regions where the radial separation between points $r$ satisfies the relationship $L<r<l$, that the statistics of the turbulence are independent of position, furthermore, that the variance and higher statistical moments depend only on the radial separation between points in the region. 


\subsubsection{Index of Refraction Structure Constant $C_{n}^{2}$}

The temperature differences in these eddies create variations in the atmospheric index of refraction. The atmospheric index of refraction can be described as the sum of the mean index and a randomly varying term.

$$
n(\vec{r})=n_{0}+n_{1}(\vec{r})
$$

where $n_{0}$ is the average index of refraction and $n_{1}(\vec{r})$ is a random fluctuation in the index that is a function of the radial distance. Turbulent eddies are volumes of atmosphere that have uniform indices of refraction. Small changes in the index of refraction accumulate over long paths through the turbulent eddies in the atmosphere and contribute to significant image degradation. The spatial distribution of these eddies is described by $\Phi_{n}(\vec{k})$; the spatial power spectral density of of $n_{1}(\vec{k})$, where $\vec{k}$ is a spatial vector with three orthogonal

components $\left(\vec{k}_{x}, \vec{k}_{y}, \vec{k}_{z}\right)$. The scale sizes of the turbulent eddies are given by $l_{x}=\frac{2 \pi}{k_{x}}, l_{y}=$ $\frac{2 \pi}{k_{y}}, l_{z}=\frac{2 \pi}{k_{z}}$. For homogeneous and isotropic turbulence, $\Phi_{n}(\vec{k})$ is a function of the scalar wave number[18],

$$
k=\sqrt{\vec{k}_{x}^{2}+\vec{k}_{y}^{2}+\vec{k}_{z}^{2}}
$$

and the isotropic scale size $l$ is related to the scalar wave number by $\frac{2 \pi}{k}$. 
This turbulence reaches its maximum in the early afternoon and varies with terrain features. The simulated images used in this study were created under the assumption that the propagation occurs over horizontally homogeneous conditions with both the scene and the imaging system immersed in a turbulent atmosphere; that the imaging path height above ground does not vary significantly and that the roughness of structural features at any one place along the propagation path look similar to those at any other spot [19].

Kolmogorov theory does not predict $\Phi_{n}(\vec{k})$ either for large scale motions, where $k<\frac{2 \pi}{L_{0}}$, or for very small scale motions where $k>\frac{2 \pi}{l_{0}}$. However, between these two regions, $\frac{2 \pi}{L_{0}} \leq k \leq$ $\frac{2 \pi}{l_{0}}$, Kolomogorov theory predicts that the spatial PSD is given by:

$$
\Phi_{n}(\vec{k})=0.033 C_{n}^{2} k^{-11 / 3}
$$

where $C_{N}^{2}$ is the index of refraction structure constant. Its dimensions are $m^{-2 / 3}$ and it has been referred to by Hufnagle as the single most important turbulent atmosphere characteristic for optical designers [20].

The atmospheric coherence radius, $r_{0}$, is commonly used to define the resolving power of an imaging system in terms of an equivalent diffraction limited system's radius. $r_{0}$ relates the index of refraction structure constant and the length of the propagation path, and is 
defined by Fried for spherical wave propagation[21][18] as

$$
r_{0}=0.185\left[\frac{4 \pi^{2}}{k^{2} \int_{0}^{L}\left(\frac{L-z}{L}\right)^{5 / 3} C_{n}^{2}(z) d z}\right]^{3 / 5}
$$

where $\lambda$ is the mean wavelength, the wave number $k=\frac{2 \pi}{\lambda}$, and $\Delta z$ is the distance from the scene to the imaging aperture. The limits of the integral are from $z=0$ at the pupil plane to the scene at $z=L$. For purposes of this study it is assumed that in Equation (1.5) that the refractive-index structure constant $C_{n}^{2}$ is constant over paths that are horizontal or at shallow angles from the aperture [19]. It is further assumed that the strength of the turbulence is such that scintillation effects can be neglected.

\subsubsection{Anisoplanatism}

Under isoplanatic conditions, the light coming from all points in the scene can be assumed to experience similar turbulence induced changes in the atmospheric refractive index, and thus similar phase aberrations. The isoplanatic angle $\theta_{0}$ is the angular separation between point sources for which the phase changes at the aperture can be considered to be significantly decorrelated. Using Fried's [22] definition for the isoplanatic angle, $\theta_{o}$,

$$
\theta_{o}=\left(2.91 k^{2} C_{N}^{2} \Delta z^{(5 / 3)}\right)^{(-3 / 5)}
$$


However, in many near-surface surveillance imaging scenarios, it is reasonable to assume that the field of view of the imaging system will subtend an angle wide enough that this assumption will not be valid. In this case we describe the viewing as anisoplanatic. Figure 1.2 shows the the effect of anisoplanatism on three separate points in the object as spherical waves propagate through two layers of turbulent atmosphere. The longer the optical path length, and the stronger the turbulence, the more severe these aberrations become, and the isoplanatic angle decreases. Increasing the size of the aperture will not improve the quality of the image under anisoplanatic conditions, and unless the seeing conditions are very favorable, anisoplanatism will play a role in most practical horizontal imaging situations. Some technique for reducing the effects of anisoplanatism is needed. The data

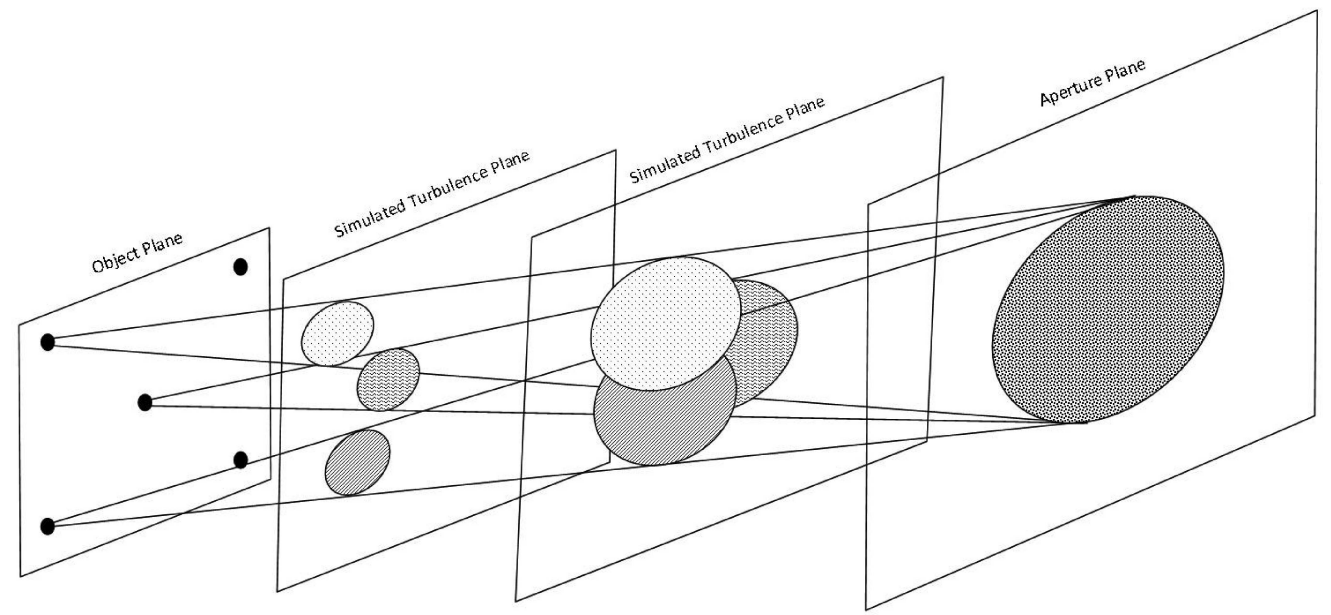

Figure 1.2: Anisoplanatism

sets simulated for this work can be categorized by the value of $C_{n}^{2}$ : for the low turbulence condition $C_{n}^{2}=2.25 \times 10^{-14} m^{(-2 / 3)}$, for the moderate condition $C_{n}^{2}=3.75 \times 10^{-14} m^{(-2 / 3)}$ and for the severe condition $C_{n}^{2}=5.25 \times 10^{-14} \mathrm{~m}^{(-2 / 3)}$. A complete description of the 
simulator used to create these data sets is available in [23].

Under isoplanatic conditions, it can be assumed that the image formation can be modeled in the spatial domain as the convolution of the atmospheric point-spread function (PSF) and the object intensity distribution.

$$
i\left(\vec{x}_{i}\right)=o\left(\vec{x}_{i}\right) * h\left(\vec{x}_{i}\right)
$$

where $\vec{x}_{i}$ is a two dimensional coordinate in the image plane, and $o\left(\vec{x}_{i}\right)$ is the image of the object predicted by geometrical optics. The assumption that the point spread function is shift-invariant does not hold under anisoplanatic conditions. Figure 1.3 (a) shows a pin cushion image that has been propagated over $10 \mathrm{~km}$ under what are considered in this work to be moderate turbulence conditions. Figure 1.3 (b) shows a point spread function from the upper left quadrant of the image, and Figure 1.3 (c) is from the lower right quadrant. The basic structure and spatial shift of both is similar, but the "corona", the area around the central bright spot is subtly different. There is some support for the conjecture that the point spread function recovered by the MFBD processing is closely related to the spatial average of the individual PSFs. Recall that the incoherent point spread function $h\left(\vec{x}_{i}\right)$ is the modulus squared of the coherent point spread function.

$$
h\left(\vec{x}_{i}\right)=\left|g\left(\vec{x}_{i}\right)\right|^{2}
$$




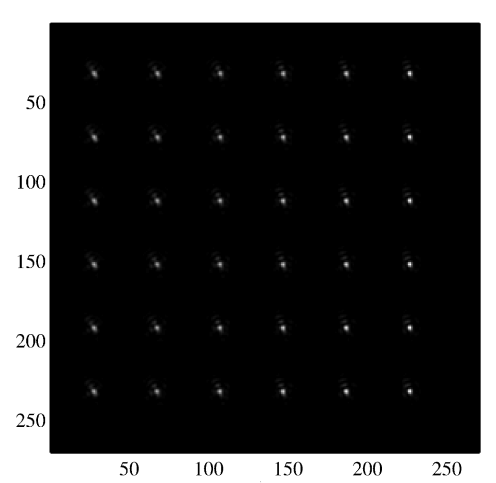

(a)

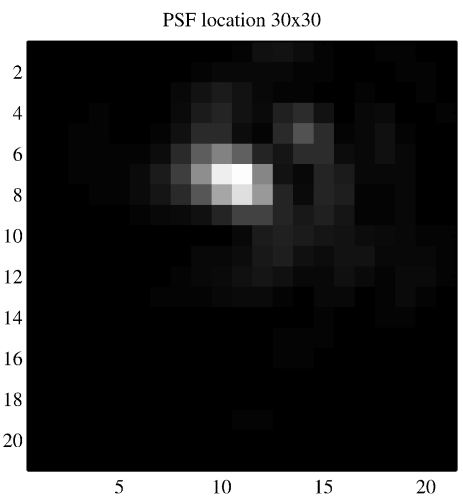

(b)

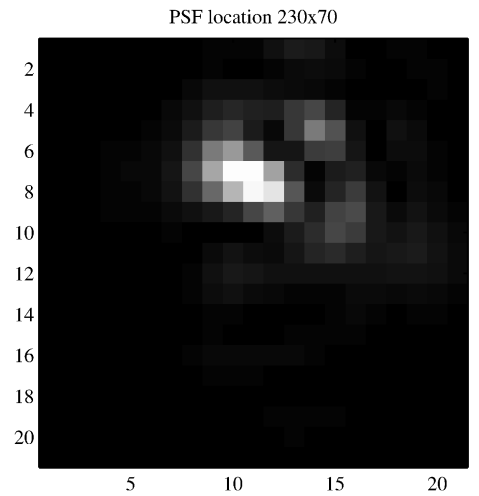

(c)

Figure 1.3: Anisoplanatic Pincushion Point Spread function. Figure (a) shows a pin cushion array of point sources corrupted under moderate turbulence condition. Figure (b) shows a 22 x 21 pixel extract, centered on the pixel location $(30,30)$. Figure (c) is a similar extract centered on location $(230,70)$

where the coherent point spread function is given by

$$
g(\vec{x})=\sum_{\overrightarrow{x_{p}}} A e^{j \theta\left(\overrightarrow{x_{p}}\right)} e^{j \frac{2 \pi}{N}\left(\vec{x} \bullet \overrightarrow{x_{p}}\right)}
$$

where $A$ is a binary aperture function whose value is zero outside the aperture, $\vec{x}_{p}$ is a two dimensional coordinate system in the aperture plane, and the $\bullet$ operator represents the inner product, and

$$
\theta\left(\overrightarrow{x_{p}}\right)=\sum \phi_{k}\left(\overrightarrow{x_{p}}\right) \alpha_{k}
$$

The phase term $\theta\left(\overrightarrow{x_{p}}\right)$ represents the accumulated phase perturbation in the aperture of the imaging system, decomposed to a linear sum of orthonormal basis functions, $\phi_{k}\left(\overrightarrow{x_{p}}\right)$, and $\alpha_{k}$ are the weighting coefficients for each term and image. The modulus of the optical 
transfer function is very small, but non-zero, for spatial frequencies greater than $\frac{r_{0}}{\lambda \Delta z}$, causing the turbulent atmosphere to act like a low pass filter, limiting its ability to resolve small features. Zernike polynomials are a convenient set of basis functions commonly used in describing classical aberrations in optical systems.

\subsubsection{Zernike Polynomials}

Zernike Polynomials are an infinite set of polynomials that are orthonormal on a unit circle [24], [25] [26], and separable functions of the radius $r$, and the azimuth, $\theta$. They are germane to this dissertation because they enable a polynomial expansion of the wave front phase term over the aperture of the simulated imaging system. The two dimensional Cartesian coordinates $\vec{x}$ can be mapped onto polar coordinates as follows:

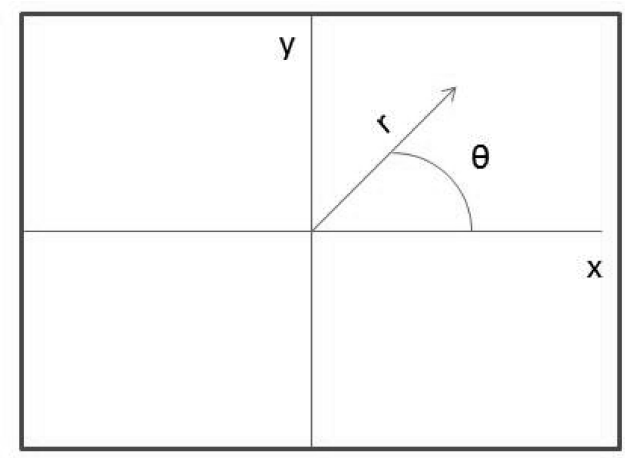

Figure 1.4: Cartesian coordinates mapped onto polar coordinates 


$$
\begin{aligned}
& x=r \cos (\theta) \\
& y=r \sin (\theta) \\
& r=\sqrt{x^{2}+y^{2}} \\
& \theta=\tan ^{-1} \frac{y}{x}
\end{aligned}
$$

$$
\begin{aligned}
& \left.\begin{array}{rl}
\phi_{k} \text { even }(r, \theta) & =\sqrt{n+1} R_{n}^{m}(r) \cos (m \theta) \\
\phi_{k} \operatorname{odd}(r, \theta) & =\sqrt{n+1} R_{n}^{m}(r) \sin (m \theta)
\end{array}\right\} m \neq 0 \\
& \phi_{k}(r, \theta)=R_{n}^{m}(r) m=0
\end{aligned}
$$

The radial function $R_{n}^{m}(r)$ is defined by

$$
R_{n}^{m}(r)=\sum_{s=0}^{\frac{(n-m)}{2}}\left\{\frac{(-1)^{s}(n-s) !}{s !\left[\frac{(n+m)}{2}-s\right] !\left[\frac{(n-m)}{2}-s\right] !}\right\} r^{n-2 s}
$$

The Zernike terms are ordered by the non-negative integers $m$, the azimuthal order, and $n$ the radial order. These satisfy the conditions that $m<n$ and $n-m$ is even. One of the advantages of Zernike polynomials as a basis set is that closed forms exist, and that the lower order terms relate to classical abberations. For example, following the ordering defined by Noll [7] the first Zernike mode $\phi_{1}$ is referred to as piston, the second and third $\phi_{2}, \phi_{3}$ are tilt in the $x$ and $y$ directions, the $\phi_{4}$ fourth is defocus, $\phi_{5}$ and $\phi_{6}$ components of astigmatism. 


\subsection{Method}

In my dissertation, I propose a method of estimating an extended object from both simulated and actual turbulent images under anisoplanatic conditions using a non-linear optimization technique. The BFGS optimization algorithm, named after is co-creators, Broyden, Fletcher, Goldfarb, and Shanno, is a quasi-Newtonian hill climbing method. The technique begins at some arbitrary initial point on a cost surface. Using the first derivative of the cost function, in this case the log-likelihood function parameterized by the object intensities $o(\vec{x})$ from Equation 1.7, and the coefficients of the Zernike polynomials $\alpha_{k}$ in Equation 1.10 that is developed in Chapter 2, the algorithm decides on a direction to move along the surface. The estimate is updated with new values for object intensities and Zernike coefficients and the process begins again. The process continues in the direction pointed by the gradient until the cost begins to increase again. Knowledge of the second derivative, the Hessian matrix, assists in determining the location of a local minimum by slowing the descent as the value of the Hessian decreases. The BFGS method does not require an explicit expression of the Hessian, because it estimates the Hessian by maintaining recent updates to the gradient. BFGS is not guaranteed to find a global minimum for the cost function. It is possible for it to become trapped in a local minimum. I will explore the performance of the estimator by varying specific parameters and compare its performance to speckle imaging techniques in order make recommendations for further investigation and investment of resources. The estimator design characteristics varied in 
this exploration are:

- Noise Model - The turbulence is assumed to follow either Gaussian statistics, or for the low light cases, the Poisson noise model

- $N_{f}$ - The number of input frames applied to the estimator. Initial estimates

- $N_{z}$ - The number of Zernike polynomial terms used to characterize the phase term of the Optical Transfer Function.

- $N_{i}$ - The number of iterations allowed to the optimization routine.

The characteristics of the input images

- Padding - The amount and nature of the padding applied to the input images. Included in this category is the application of any windowing to the image and padding.

- Turbulence - Estimator performance is evaluated over three levels of turbulence strength described in this work as Low, Moderate and Severe, that result in correspondingly blurred images.

- Ilumination - estimator performance is evaluated under three increasingly photon starved conditions, Mean Photon Rate $2 \times 10^{6}, 1 \times 10^{6}$, and $5 \times 10^{5}$. Performance at each light level is evaluated under all three turbulence conditions. 


\subsection{Summary of Key Results}

I found that the MFBD reconstructed images showed significant improvement in mean squared error(MSE) compared to the average MSE. For the Gaussian noise model case the improvement in MSE was 40 percent for the low turbulence case using as few as 14 images and 30 Zernike coefficients in the reconstruction, 25 percent for moderate turbulence, and 36 percent for severe turbulence case. Parametric study of the number of Zernike terms useful in reconstruction showed that using more that 30 terms did not meaningfully improves the MSE. For the Poisson noise model the estimator required a minimum of 50 frames to achieve significant improvement over the average MSE. Reconstructed objects showed as much as 38 percent improvement in MSE using 175 frames and 30 Zernike coefficients in the reconstruction. These results were published in Optical Engineering. [27] When compared to the Bispectrum technique, the MFBD estimator did not perform as well either in overall MSE or in processing time. However, from a qualitative point of view, the reconstructed images present a better appearance to the human eye than those reconstructed using Bispectrum. The hybrid Bispectrum-MFBD estimator use of a Bispectrum as an initial guess for the BFGS estimator provided improvement in the overall MSE as well as smaller variance, regardless of how many images were used in the reconstruction. Portions of these results have been published in the Proceedings of the 2013 SPIE Defense Systems and Sensing conference and are currently under peer review with the Optical Engineering Journal. 


\subsection{Organization}

The remainder of this paper is comprised of two journal articles one of which has been accepted for publication by the editors of SPIE Optical Engineering and the other is under peer review with the same journal. Chapter 2 refers to "Reconstruction of Long Horizontal-Path Images under Anisoplanatic conditions Using Multi-Frame Blind Deconvolution" published in Optical Engineering, July 2013. In the paper I describe the algorithm and use it to reconstruct images under both Gaussian and Poisson noise models under a variety of turbulence and illumination conditions. Chapter 3 is derived from "A Comparison of the Mean Squared Error Performance of Speckle and MFBD Image Reconstruction Techniques Under Anisoplanatic, Long Horizontal-Path Imaging." which has been accepted for review by Optical Engineering. In it I compare the MFBD estimator performance to the Bispectrum technique for image reconstruction. Chapter 4 includes a demonstration of the MFBD estimator's performance against field data. In Chapter 5 I present a strategy to provide the MFBD with an initial estimate based on the output of the bispectrum estimator. The content of Chapters 34 , and 5 have been accepted by Optical Engineering pending peer review. Chapter 6 summarizes the findings in my body of work and sets the stage for further research. 


\section{Chapter 2}

\section{Reconstruction of Long Horizontal-Path}

\section{Images under Anisoplanatic conditions}

\section{Using Multi-Frame Blind Deconvolution}

${ }^{1}$ The material contained in this chapter was previously published "Reconstruction of long horizontal-path images under anisoplanatic conditions using multiframe blind deconvolution," Optical Engineering, vol. 52, August 2013. 


\subsection{Introduction}

This chapter describes a method of jointly estimating object intensities and imaging system point spread functions from simulated anisoplanatic images that have been corrupted by atmospheric turbulence. The image model that forms the foundation of this estimator is that of a linear shift invariant point spread function and a deterministic object. It is conjectured that anisoplanatic effects of the turbulent atmosphere are compensated by the estimator by reconstructing a spatialy averaged point spread function. Bos' [23] work using cross-spectrum and bispectrum phase reconstructions points to this potential solution. Carrano[15] has also published work in this area that neglects the anisoplanatic effects. This investigation will be the subject of another paper. The method developed in this chapter is applied to three sets of images corrupted by different levels of turbulence, and the effectiveness is assessed by calculating the MSE between the resulting recovered object and the diffraction limited image.

The goal of this chapter is to demonstrate the use of a parameterized, multi-frame-blind-deconvolution (MFBD) technique to reconstruct an object estimate from a set of simulated anisoplanatic images, and examine the mean square error (MSE) performance of the estimator as the parameters are varied. In this chapter I examine estimator performance as the number of frames used in the estimation is varied, and as the number of Zernike polynomial coefficients used to characterize the phase term of the 
point-spread function described in section 1.1.2 are varied under the assumption of both Gaussian and Poisson noise distributions. Futhermore, the performance of the estimator is characterized as various levels of additive Gaussian noise are added to the input images.

I now describe the MFBD algorithm for the Gaussian and Poisson noise models. In MFBD the input is a set of turbulence corrupted images. In a stack of $K$ turbulence-corrupted, but measurement noise-free images, the $k_{t h}$ image can be described as the convolution of an unchanging object in space convolved with the point spread function (PSF) of the optical system $h\left(\vec{x}_{i}\right)$. Mathematically this can be expressed as [28]

$$
i\left(\vec{x}_{i}\right)=o\left(\vec{x}_{i}\right) * h\left(\vec{x}_{i}\right)
$$

Where $\star$ represents the two dimensional convolution operator. The expression $i_{k}\left(\vec{x}_{i}\right)$ represents the $k_{t h}$ noiseless image, $o\left(\vec{x}_{i}\right)$ is the irradiance distribution of the object in the image plane predicted by geometrical optics, and $h_{k}\left(\vec{x}_{i}\right)$ is the $k_{t h}$ incoherent point spread function.

The PSF is the modulus squared of the coherent impulse response $\left|g_{k}\left(\vec{x}_{i}\right)\right|^{2}$, which is in turn the inverse Fourier transform of the generalized pupil function.

$$
g\left(\vec{x}_{i}\right)=\sum_{\overrightarrow{x_{p}}} A e^{j \theta\left(\vec{x}_{p}\right)} e^{j \frac{2 \pi}{N}\left(\vec{x}_{i} \bullet \overrightarrow{x_{p}}\right)}
$$

where $A$ is a binary aperture function whose value is zero outside the aperture, $\overrightarrow{x_{p}}$ is a two 
dimensional coordinate system in the aperture plane, and the $\bullet$ operator represents the inner product, and

$$
\theta\left(\vec{x}_{p}\right)=\sum \phi_{k}\left(\overrightarrow{x_{p}}\right) \alpha_{k} .
$$

The phase term $\theta\left(\overrightarrow{x_{p}}\right)$ represents the accumulated phase perturbation in the aperture of the imaging system decomposed to a linear sum of orthonormal basis functions, $\phi_{k}\left(\overrightarrow{x_{p}}\right)$, and $\alpha_{k}$ are the weighting coefficients for each term and image.

$$
\tilde{\phi}_{k}\left(\overrightarrow{x_{p}}, \vec{\alpha}\right) \approx \sum_{j=1}^{J} \alpha_{j, k} \phi_{j}\left(\overrightarrow{x_{p}}\right)
$$

where the coefficients $\alpha_{j, k}$ serve to weight the basis functions $\phi_{j}\left(\vec{x}_{p}\right)$. Zernike polynomials are a common set of orthonormal basis functions used to characterize both fixed and random aberrations in imaging systems and are frequently used to describe turbulence effects on imaging [21]. I assume that the simulated images $d_{k}\left(\vec{x}_{i}\right)$ are a series of short-exposure turbulence-corrupted images, where the object in the scene remains constant but the phases, $\phi_{k}\left(\vec{x}_{p}\right)$ associated with each PSF are random in each image frame. This allows the expression of the generalized pupil function as a function of both the spatial 
frequency and the vector of Zernike coefficients $\overrightarrow{\alpha_{j, k}}$

$$
H_{k}\left(\vec{f}, \vec{\alpha}_{j, k}\right)=|H(\vec{f})| e^{j \phi_{k}(\vec{f}, \vec{\alpha})}
$$

which allows me to approximate the $k_{t h}$ aberrated point spread function as a weighted vector of Zernike polynomials. In non-blind deconvolution problems, the data collected, $d_{k}\left(\vec{x}_{i}\right)$, is used with a known point spread function $h_{k}\left(\vec{x}_{i}\right)$ to determine $o\left(\vec{x}_{i}\right)$. In blind deconvolution, we are given $d_{k}\left(\vec{x}_{i}\right)$ and use that information to estimate both the object, $o\left(\vec{x}_{i}\right)$ and the point spread function $h_{k}\left(\vec{x}_{i}\right)$ jointly. There is no closed form solution to the problem of jointly estimating an object and the aberration parameters for each image frame. Hence, an iterative approach is needed to find the object pixel intensities and Zernike coefficients that are most likely to have resulted in the simulated data for each image. In section 2.1.2 I describe two such approaches; one based on a Gaussian noise model and one based on a Poisson noise model.

\subsubsection{Data Set}

It is common to simulate the effects of the turbulent atmosphere by placing layers of uniform turbulence between the object and the imaging system. The data set consisting of 1000 simulated turbulent images used in this paper was created using the "Lenna" [29] standard test image. Five Kolmogorov phase screens were generated. The image 
Table 2.1

Atmospheric Simulation Turbulence Conditions

\begin{tabular}{|r|c|c|c|c|}
\hline \multicolumn{5}{|c|}{ Atmospheric Turbulence Parameters } \\
\hline $\begin{array}{r}\text { Severity } \\
\text { condition }\end{array}$ & $\begin{array}{c}C_{N}^{2} \\
\left(m^{(-2 / 3)}\right)\end{array}$ & $\begin{array}{c}r_{0} \text { spherical case } \\
(\mathrm{cm})\end{array}$ & $\begin{array}{c}\theta_{0} \\
(\mu \text { radians })\end{array}$ & $\begin{array}{c}\theta_{0} \\
\text { (pixels) }\end{array}$ \\
\hline Low & $2.25 \times 10^{-14}$ & 3.33 & 10.8 & 4 \\
\hline Moderate & $3.75 \times 10^{-14}$ & 2.45 & 7.75 & 3 \\
\hline High & $5.25 \times 10^{-14}$ & 2.01 & 6.63 & 2 \\
\hline
\end{tabular}

was propagated over a distance of 1000 meters. Light from each object pixel was projected through the phase screens, in turn, at 200 meter separations using a geometric optics approach to account for the effects of anisoplanatism. Phase errors accumulating from each screen are combined at the pupil to create a turbulence-degraded point spread function(PSF). Each of the PSFs is then scaled by the object pixel intensities to create a turbulence corrupted image for low, moderate, and severe turbulence conditions. Parameters for the simulated imaging system include a $10 \mathrm{~cm}$ aperture with a $358 \times 358$ pixel detector and a $0.7 \mathrm{~mm}$ pixel pitch. A fuller description of the simulator used to create this data set is available in [23].

For the conditions simulated here, a single pixel in the simulated imaging system captures 2.79 $\mu$ radians. Expressing the $\theta_{0}$ values for the low, medium and severe turbulence conditions of the simulation, we see that the isoplanatic patch covers 4,3 and 2 pixels in the simulated imaging system. 


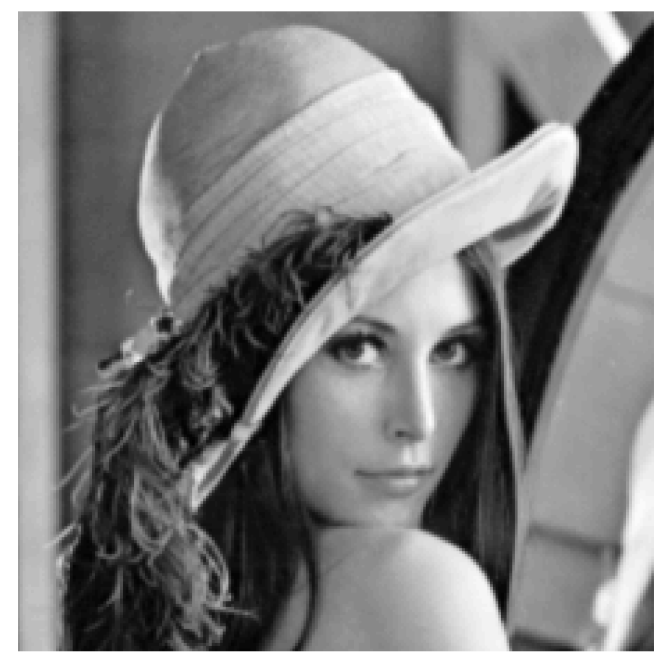

(a)

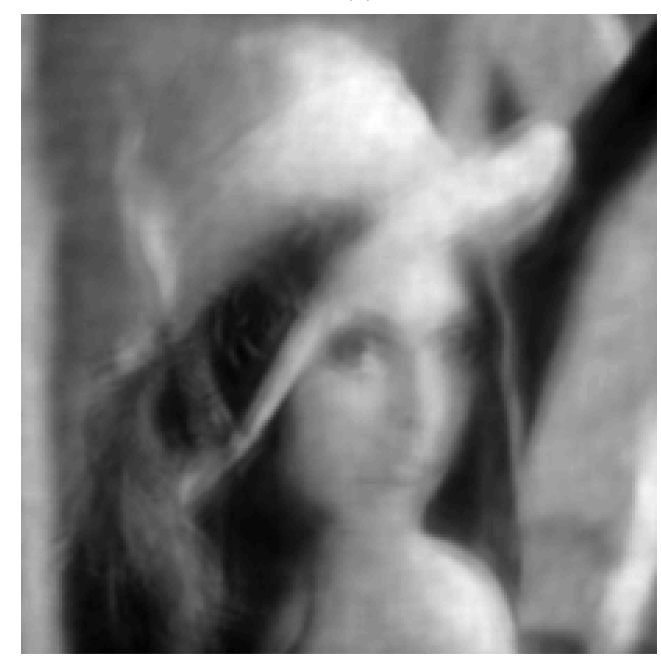

(c)

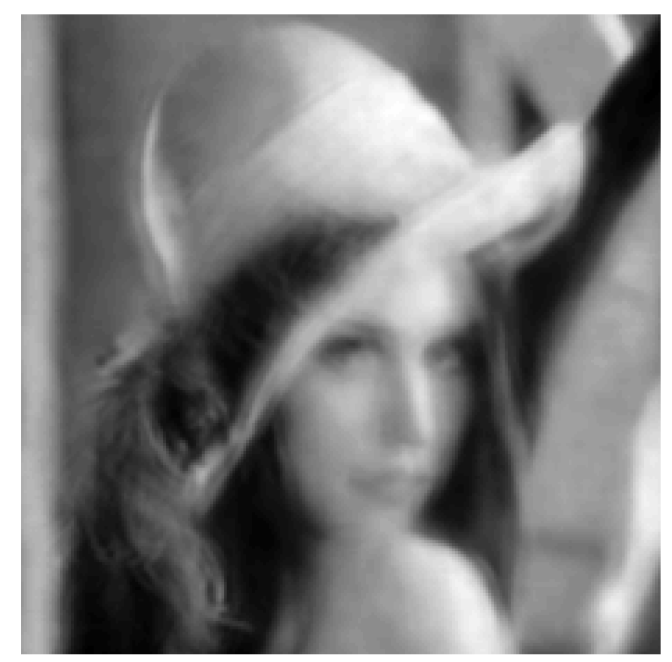

(b)

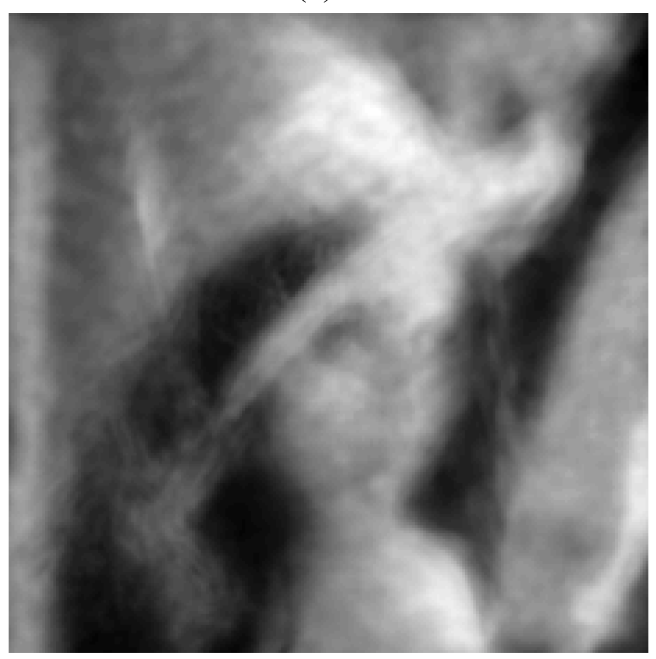

(d)

Figure 2.1: Horizontal Imaging Simulator Output. Single image representative samples of the horizontal image simulator output (a) Diffraction-Limited Image, (b) Image for $C_{N}^{2}=2.25 \times 10^{-14} m^{(-2 / 3)}$, (c) Image for $C_{N}^{2}=3.25 \times 10^{-14} m^{(-2 / 3)}$, (d) Image for $C_{N}^{2}=5.25 \times$ $10^{-14} m^{(-2 / 3)}$. 


\subsubsection{Gaussian Noise Model MFBD}

Extending the image formation equations described previously in Eqns. 2.1 through 2.4, a set of images can be described that have been corrupted by additive Gaussian noise.

$$
d_{k}\left(\vec{x}_{i}\right)=i\left(\vec{x}_{i}\right)+n_{k}\left(\vec{x}_{i}\right)=o\left(\vec{x}_{i}\right) * h_{k}\left(\vec{x}_{i}, \vec{\alpha}_{k}\right)+n_{k}\left(\vec{x}_{i}\right)
$$

where $n_{k}\left(\vec{x}_{i}\right)$ represents an additive noise term characterized by an independent, identically distributed Gaussian random variable with zero mean and variance $\sigma^{2}$. Using a Gaussian measurement noise model, each image $d_{k}\left(\vec{x}_{i}\right)$ is a random variable with a Gaussian probability density function. The pdf of $d_{k}\left(\vec{x}_{i}\right)$ is parameterized by the object intensities $o\left(\vec{x}_{o}\right)$ and the vector of aberration weighting coefficients $\vec{\alpha}_{k}$.

$$
p\left[d_{k}\left(\vec{x}_{i}\right) ; i\left(\vec{x}_{i}, \vec{\alpha}_{k}\right)\right]=\frac{1}{\left(2 \pi \sigma_{n}^{2}\right)^{1 / 2}} \exp \left\{-\frac{\left[d_{k}\left(\vec{x}_{i}\right)-i_{k}\left(\vec{x}_{i}, \vec{\alpha}_{k}\right)\right]^{2}}{2 \pi \sigma_{n}^{2}}\right\}
$$

and the likelihood of the complete data set consisting of all the pixel intensities in all the corrupted images is given by

$$
p\left[\left\{d_{k}\left(\vec{x}_{i}\right)\right\} ; i\left(\vec{x}_{i}, \vec{\alpha}_{k}\right)\right]=\prod_{k=1}^{K} \prod_{x \varepsilon \chi} \frac{1}{\left(2 \pi \sigma_{n}^{2}\right)^{1 / 2}} \exp \left\{-\frac{\left[d_{k}\left(\vec{x}_{i}\right)-i_{k}\left(\vec{x}_{i}, \vec{\alpha}_{k}\right)\right]^{2}}{2 \pi \sigma_{n}^{2}}\right\}
$$

The natural $\log$ of Eqn. (2.8) is taken in order to make the analysis more tractable, resulting in a summation rather than products and neglecting a constant term, yields the 
log-likelihood function

$$
L\left(i\left(\vec{x}_{i}, \vec{\alpha}_{k}\right)\right)=-\sum_{k=1}^{K} \sum_{x \varepsilon \chi}\left[d_{k}\left(\vec{x}_{i}\right)-i\left(\vec{x}_{i}, \vec{\alpha}_{k}\right)\right]^{2}
$$

Although an analytic form of the Hessian is not required, the L-BFGS optimization used to maximize the likelihood function Eqn. 2.9 is more efficient if an analytic form of the gradient is provided. With respect to the pixel intensities the gradient of the Gaussian Log-Likelihood function can be represented as:

$$
\frac{\partial}{\partial o} L\left(i\left(\vec{x}_{i}, \vec{\alpha}\right)\right)=2 \sum_{k=1}^{K} \sum_{x \varepsilon \chi}\left[d_{k}\left(\vec{x}_{i}\right)-i_{k}\left(\vec{x}_{i}\right)\right] \frac{\partial}{\partial o} i_{k}\left(\vec{x}_{i}\right)
$$

and the derivative of $i_{k}\left(\vec{x}_{i}\right)$ with respect to the object is given by

$$
\frac{\partial}{\partial o} i_{k}\left(\vec{x}_{i}\right)=\frac{\partial}{\partial o} \sum_{\vec{o}_{o}} h\left(\vec{x}_{i}-\vec{x}_{o}\right) o\left(\vec{x}_{i}\right)=h\left(\vec{x}_{i}-\vec{x}_{o}\right)
$$

where $h\left(x_{i}-x_{o}\right)$ is the incoherent point spread function. The gradient with respect to the Zernike coefficients is

$$
\begin{aligned}
& \left.\frac{\partial}{\partial \vec{\alpha}} L\left(i\left(\vec{x}_{i}, \vec{\alpha}\right)\right)=2 \sum_{k=1}^{K} \sum_{x \varepsilon \chi}\left[d_{k}\left(\vec{x}_{i}\right)\right)-\left(i_{k}\left(\vec{x}_{i}\right)\right)\right] \frac{\partial}{\partial \vec{\alpha}} i_{k}\left(\vec{x}_{i}, \vec{\alpha}_{k}\right) . \\
& \frac{\partial}{\partial \vec{\alpha}} i_{k}\left(\vec{x}_{i}, \vec{\alpha}_{k}\right)=\frac{\partial}{\partial \vec{\alpha}}\left(\sum_{\overrightarrow{x_{o}}} h\left(\vec{x}_{i}-\vec{x}_{o}\right) o\left(\vec{x}_{i}\right)\right)=\sum_{\overrightarrow{x_{o}}} \frac{\partial}{\partial \vec{\alpha}}\left(h\left(\vec{x}_{i}-\vec{x}_{o}\right) o\left(\vec{x}_{i}\right)\right)
\end{aligned}
$$


Since the object $o\left(\vec{x}_{o}\right)$ is constant with respect to the Zernike coefficients, Equation 2.13 reduces to

$$
\sum_{\vec{x}_{o}} \frac{\partial}{\partial \vec{\alpha}}\left(h\left(\vec{x}_{i}-\vec{x}_{o}\right) o\left(\vec{x}_{i}\right)\right)=\sum_{\vec{x}_{o}}\left(\frac{\partial}{\partial \vec{\alpha}}\left(h\left(\vec{x}_{i}-\vec{x}_{o}\right)\right) o\left(\vec{x}_{i}\right)\right.
$$

From equations 1.8, 1.9, and 1.10, we can express the derivative of the incoherent point spread function with respect to the $\alpha_{k}$ s in terms of the coherent point spread function,

$$
\frac{\partial}{\partial \vec{\alpha}}\left(h\left(\vec{x}_{i}-\vec{x}_{o}\right)\right)=\frac{\partial}{\partial \vec{\alpha}}\left|g\left(\vec{x}_{i}\right)\right|^{2}=\frac{\partial}{\partial \vec{\alpha}} g\left(\vec{x}_{i}\right) g^{*}\left(\vec{x}_{i}\right)=\left[\frac{\partial}{\partial \vec{\alpha}} g\left(\vec{x}_{i}\right)\right] g^{*}\left(\vec{x}_{i}\right)+g\left(\vec{x}_{i}\right)\left[\frac{\partial}{\partial \vec{\alpha}} g^{*}\left(\vec{x}_{i}\right)\right] .
$$

where $*(\bullet)$ represents the complex conjugate of the function in parenthesis. Equation 2.15 can be represented as

$$
2 \Re\left\{\left[\frac{\partial}{\partial \vec{\alpha}} g\left(\vec{x}_{i}\right)\right] g^{*}\left(\vec{x}_{i}\right)\right\},
$$

where $\Re(\bullet)$ takes the real part of a complex value.

$$
\frac{\partial}{\partial \vec{\alpha}} g\left(\vec{x}_{i}\right)=\frac{\partial}{\partial \vec{\alpha}}\left[\sum_{\overrightarrow{x_{p}}} A e^{j \theta\left(\overrightarrow{x_{p}}\right)} e^{j \frac{2 \pi}{N}\left(\vec{x}_{i} \bullet \vec{x}_{p}\right)}\right]=\sum_{\overrightarrow{x_{p}}}\left[j \frac{\partial \theta\left(\overrightarrow{x_{p}}\right)}{\partial \vec{\alpha}} A e^{j \theta\left(\vec{x}_{p}\right)} e^{j \frac{2 \pi}{N}\left(\vec{x}_{i} \bullet \overrightarrow{x_{p}}\right)}\right]
$$




$$
\frac{\partial \theta\left(\overrightarrow{x_{p}}\right)}{\partial \vec{\alpha}}=\frac{\partial}{\partial \vec{\alpha}} \phi_{k}\left(\overrightarrow{x_{p}}\right) \alpha_{k}=\phi_{k}\left(\overrightarrow{x_{p}}\right)
$$

Combining the results of Equations 2.18, 2.17, 2.16 yields the final result

$$
\frac{\partial}{\partial \vec{\alpha}} i_{k}\left(\vec{x}_{i}, \vec{\alpha}_{k}\right)=\sum_{\vec{x}_{i}} 2 \Re\left\{\left[\sum_{\overrightarrow{x_{p}}}\left[j \phi_{k}\left(\vec{x}_{p}\right) A e^{j \theta\left(\vec{x}_{p}\right)} e^{j \frac{2 \pi}{N}\left(\vec{x}_{i} \bullet \vec{x}_{p}\right)}\right]\right] g^{*}\left(\vec{x}_{i}\right)\right\} o\left(\vec{x}_{i}\right)
$$

\subsubsection{Poisson noise model MFBD}

Not all images are taken in full daylight. At low light levels, photon noise may dominate image frames. This is often characterized by a speckled quality of the images. Photon noise in images is described by modeling the number of photons detected in an image frame at each pixel as a Poisson random variable with a mean photon count rate $\lambda$, which is proportional to average pixel intensity. For this simulation the number of photons detected at each detector pixel is assumed to be an independent, Poisson distributed random variable with a mean rate given by a noiseless diffraction-limited image $g\left(\vec{x}_{i}\right)$. The random nature

of the point spread function is neglected. The probability of detecting $d_{k}\left(\vec{x}_{i}\right)$ photons at a specific pixel location is given by

$$
p\left[d_{k}\left(\vec{x}_{i}\right)\right]=\frac{g\left(\vec{x}_{i}\right)^{d_{k}\left(\vec{x}_{i}\right)} e^{\left[-g_{k}\left(\vec{x}_{i}\right)\right]}}{d_{k}\left(\vec{x}_{i}\right) !}
$$


The distribution over the entire set of pixel locations $d_{k}$ is given by

$$
p\left[\left\{d_{k}\right\}\right]=\prod_{k=1}^{K} \prod_{x \varepsilon \chi} \frac{g\left(\vec{x}_{i}\right)^{d_{k}\left(\vec{x}_{i}\right)} e^{\left[-g_{k}\left(\vec{x}_{i}\right)\right]}}{d_{k}\left(\vec{x}_{i}\right) !}
$$

As before, taking the natural log yields a modified log-likelihood function

$$
L_{\text {poisson }} f\left(\vec{x}_{i}, \vec{\alpha}\right)=-\sum_{k=1}^{K} \sum_{x \varepsilon \chi}\left[d_{k}\left(\vec{x}_{i}\right) \ln \left(g_{k}\left(\vec{x}_{i}\right)\right)-g_{k}\left(\vec{x}_{i}\right)\right]-\sum_{k=1}^{K} \sum_{x \varepsilon \chi} d_{k}\left(\vec{x}_{i}\right),
$$

where the last term is a constant and can be neglected. Taking the derivative with respect to the pixel intensities, the gradient of the Poisson Log-Likelihood function can be represented as:

$$
\frac{\partial}{\partial f} L_{\text {Poisson }} f\left(\vec{x}_{i}, \vec{\alpha}\right)=\sum_{k=1}^{K} \sum_{x \varepsilon \chi}\left[\frac{d_{k}\left(\vec{x}_{i}\right)}{g_{k}\left(\vec{x}_{i}\right)}-1\right] \frac{\partial}{\partial f} g_{k}\left(\vec{x}_{i}\right)
$$

With respect to the Zernike coefficients the gradient of the Poisson Log-Likelihood function can be represented as:

$$
\frac{\partial}{\partial \vec{\alpha}} L_{\text {Poisson }} f\left(\vec{x}_{i}, \vec{\alpha}\right)=\sum_{k=1}^{K} \sum_{\chi}\left[\frac{d_{k}\left(\vec{x}_{i}\right)}{g_{k}\left(\vec{x}_{i}\right)}-1\right] \frac{\partial}{\partial \vec{\alpha}} g_{k}\left(\vec{x}_{i}\right) .
$$




\subsection{Methods}

The simulations assume that the propagation occurs over horizontally homogeneous conditions with both the object and the imaging system immersed in a turbulent atmosphere. Furthermore, I assume that the height above ground does not vary significantly and $C_{n}^{2}$ is constant over the propagation path [19]. I assume that the simulated data has effectively frozen the turbulence at the moment the turbulent image is created. Prior to applying the simulated turbulent images to the reconstruction algorithm, they must be recentered as tilt is not estimated in the MFBD algorithm. This was accomplished by using a correlation filter to compare each image in the stack to an ensemble average image and then shifting the turbulent image to recenter it. In order to reduce edge effects in the reconstructed object, each frame of the data set was preprocessed to pad the centered image by replicating the edges of the image outward and then adding a border of zeros. The abrupt transitions artificially introduced by the padding process can result in high spatial frequency components that are sometimes mitigated by the application of spatial filters. Using fifteen frames in the reconstruction, the image stack was padded and then a Tukey [30] tapered filtered applied to the image. Both the tapered and untapered images were applied to the estimator. The elapsed processing time and mean square error of the reconstructed object were determined with the estimator limited to 20 iterations. The amount of padding for subsequent processing was determined by examining the effect on processing time and the mean square error as the amount of padding was varied. All subsequent processing was 
Table 2.2

Selection of image padding

\begin{tabular}{|c|c|c|c|c|c|}
\hline \multicolumn{5}{|c|}{ Padding, Filter vs MSE and Elapsed time } \\
\hline $\begin{array}{c}\text { Pixels } \\
\text { repeat + zeros }\end{array}$ & \multicolumn{2}{|c|}{ w/o filter } & \multicolumn{2}{|c|}{ w/ filter } & total image size \\
\hline $8+5$ & 585 & 181 & 712 & 166 & 256 \\
\hline $9+5$ & n/a & n/a & 586 & 306 & 258 \\
\hline $10+5$ & 586 & 221 & 625 & 202 & 260 \\
\hline $15+5$ & 586 & 252 & 593 & 230 & 270 \\
\hline $20+5$ & 583 & 268 & 595 & 276 & 280 \\
\hline $25+5$ & 588 & 392 & 591 & 361 & 290 \\
\hline
\end{tabular}

accomplished by padding each recentered turbulent image but without tapering it. The images are applied to the estimator with 8 replicated pixels followed by 5 zero pixels at the margins of each image, bringing the total size of the image to $256 \times 256$ pixels. Figure 2.2 (a) shows the image prior to padding, and Fig. 2.2 (b) shows the image after padding. These results are summarized in Table 2.2.

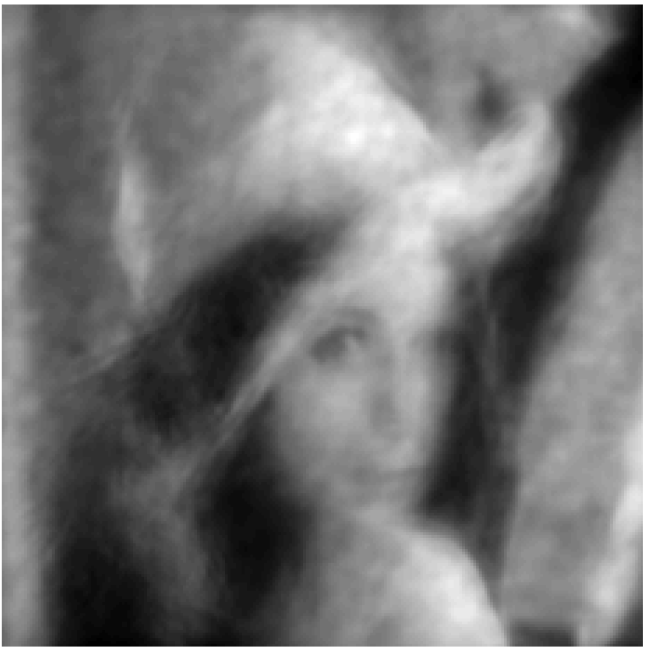

(a)

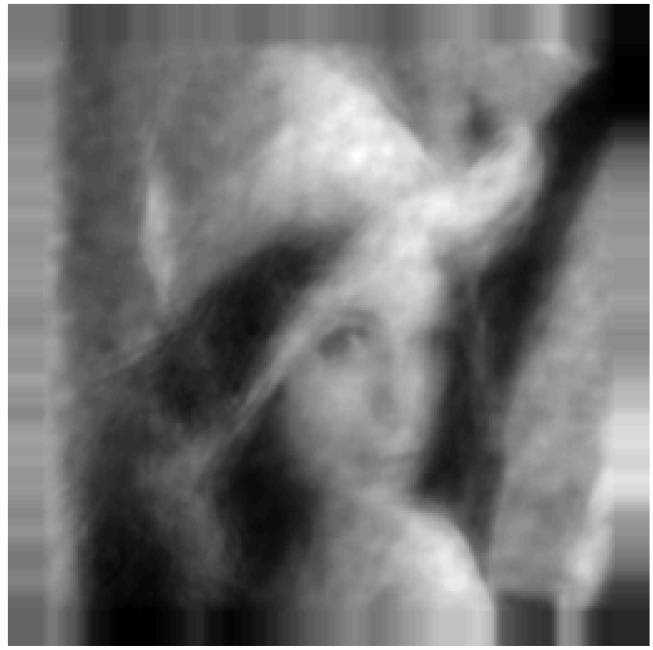

(b)

Figure 2.2: Padding Example 
Table 2.3

Input Object and Zernike Coefficients. The initial guess at the object estimate is stripped into a vector with the intensities at the beginning and the initial guess at each input image's Zernike coefficients follow.

\begin{tabular}{|l|c|c|c|c|c|c|c|c|c|c|c|c|}
\hline \multicolumn{10}{|c|}{ Estimator Input Parameters } \\
\hline $\bar{o}_{1}$ & $\bar{o}_{1}$ & $\ldots$ & $\bar{o}_{N^{2}}$ & $\hat{\alpha}_{1,1}$ & $\hat{\alpha}_{1,2}$ & $\ldots$ & $\hat{\alpha}_{1, J}$ & $\ldots$ & $\hat{\alpha}_{K, 1}$ & $\hat{\alpha}_{K, 2}$ & $\ldots$ & $\hat{\alpha}_{K, J}$ \\
\hline
\end{tabular}

\subsubsection{L-BFGS Optimization}

The cost functions in Equations 2.9 and 2.22 are parameterized by the object pixel intensities and aberration coefficients, and are applied to a non-linear optimization MATLAB [31] routine called L-BFGS [32] to find the object and aberration coefficients most likely to have produced the images that were simulated in the data set. The intensities at each pixel location in each image are vectorized. The vectorized initial guesses for each image's of Zernike polynomial coefficients are appended to the end of the vector on image intensities formatted as shown in Table 2.3 . I am jointly processing all images and all Zernike coefficients, thus for a data set of $K, N \times N$ images, using $J$ Zernike polynomial terms, there will be $N^{2}+J \times K$ parameters that must be jointly estimated. The optimization routine will return a vector of the reconstructed object's intensities, followed by the estimate of the Zernike coefficients for each frame of the input stack as shown in Table 2.4. Optimization over such a large parameter space is impractical using conventional optimization techniques. To make the optimization tractable I used the limited-memory Broyden-Fletcher-Goldfarb-Shanno (L-BFGS) method to process the images. L-BFGS 


\section{Table 2.4}

Vectorized Reconstructed Object and Zernike Coefficients. The estimate is returned as a vector with the estimated object pixel intensities at the beginning and the estimate of each input image's Zernike coeffiecients at the end.

\begin{tabular}{|l|l|l|l|l|l|l|l|l|l|l|l|l|}
\hline \multicolumn{10}{|c|}{ Output Object Estimate and Zernike Coefficient Vector } \\
\hline$\hat{o}_{1}$ & $\hat{o}_{1}$ & $\ldots$ & $\hat{o}_{N^{2}}$ & $\hat{\alpha}_{1,1}$ & $\hat{\alpha}_{1,2}$ & $\ldots$ & $\hat{\alpha}_{1, J}$ & $\ldots$ & $\hat{\alpha}_{K, 1}$ & $\hat{\alpha}_{K, 2}$ & $\ldots$ & $\hat{\alpha}_{K, J}$ \\
\hline
\end{tabular}

[32] is a quasi-Newtonian, "hill-climbing" technique that begins with an initial guess at a solution for $o\left(\vec{x}_{i 0}\right)$ and then proceeds along a line in the direction $d_{k}$ pointed to by the gradient of the objective function evaluated at each pixel location. One of the drawbacks to searching along the gradient is the need for the Hessian $\nabla^{2} f(\vec{x})$ to prevent the estimate from hopping back and forth across the sides of the valley. The limited memory form of the BFGS does not require an explicit expression for $\nabla^{2}$. It estimates the value of the Hessian matrix by maintaining the last few updates of $f(\vec{x})$ and $\nabla f(\vec{x})$. Quasi-Newtonian line search optimization can quickly converge to a local minimum for cost functions, but there is no guarantee that that minimum is a global minimum. In processing the initial object estimate applied to the estimator was the average of all the frames used in the trial.

Its necessary to provide the estimator with a stopping criterion. Using the low condition data set with the number of frames set to 5, 15 and 35, the log-likelihood function value was monitored during reconstruction. The results are shown in Figure 2.3. Regardless of the number of frames used in the reconstruction, assuming one call to the likelihood function per iteration, the reconstruction is essentially complete after 10 iterations. For the image reconstruction processing in this paper, the number of iterations was limited to 25 . 


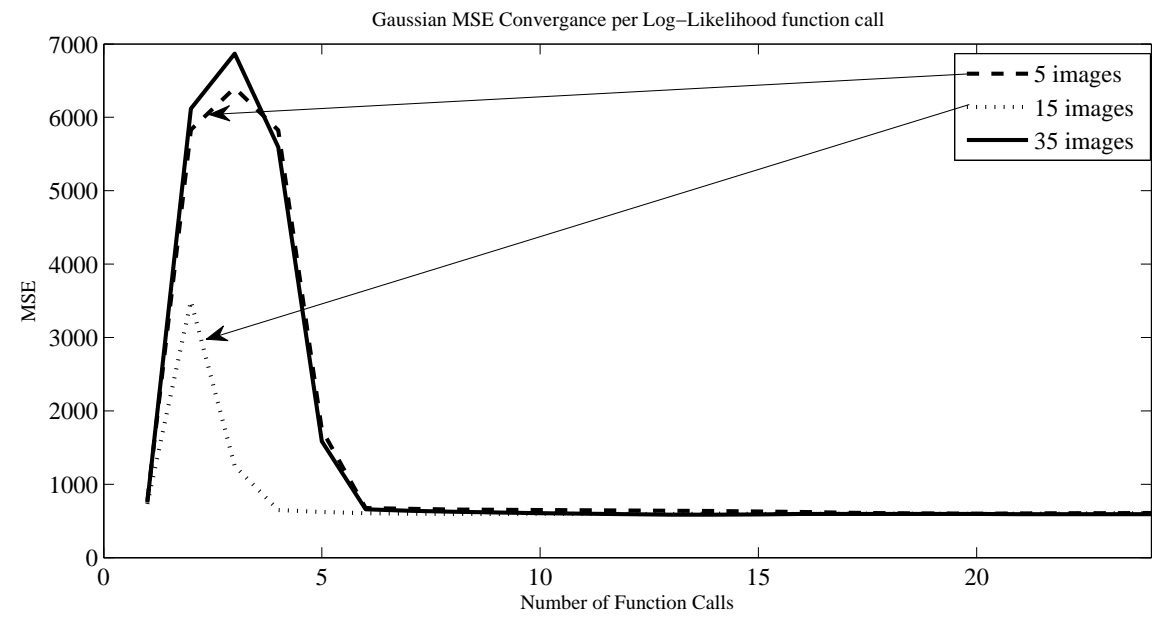

Figure 2.3: Gaussian MSE vs Number of Function Calls

\subsubsection{Reconstruction Processing}

Reconstruction processing begins by selecting images from the complete data set in groups of $K=2,4,6,8,10,12,14,16,18,20,22,23$ and 25 frames and then incrementing that group size through the entire data set. At each increment an initial guess at the object $o\left(\vec{x}_{i 0}\right)$ and phase parameters $\alpha_{i}$ is provided to the optimization routine. This initial guess is always the average of the $K$ frames being used in the estimate. The Zernike coefficients provided as an initial guess are random Gaussian numbers with a mean of 0.5 and unity variance.

The recovered image was compared to the diffraction limited image and the mean square error determined. The mean square error is averaged over all pixels and determined as follows

$$
M S E=\frac{1}{K} \sum_{K}\left(\sum_{x_{i}} \frac{\left(o\left(\vec{x}_{i}\right)-\hat{o}\left(\vec{x}_{i}\right)\right)^{2}}{N^{2}}\right)
$$


where $o\left(\vec{x}_{i}\right)$ is the normalized diffraction limited image, and $\hat{o}\left(\vec{x}_{i}\right)$ is the current normalized estimate of the object and $N^{2}$ is the total number of pixels in the image. Then the image stack was incremented to begin processing the next group of $K$ turbulent images and the process repeated. When the entire data set has been processed, the average of the vector of MSE's for images processed $K$ at a time was calculated.

\subsubsection{Number of Zernike Terms Needed in the Optimization Process}

Recovering a common object estimate from the stack of degraded images is computationally intense regardless of the method used. Using more Zernike polynomial terms requires more variables to be estimated, and longer processing will result. Fig. 2.4 (a) shows the processing time required for a fixed number of frames when the number of Zernike polynomial terms are varied, Fig.2.4(b) shows how the processing time varies for a fixed number of Zernike coefficients as the number of input images is varied. Of greater

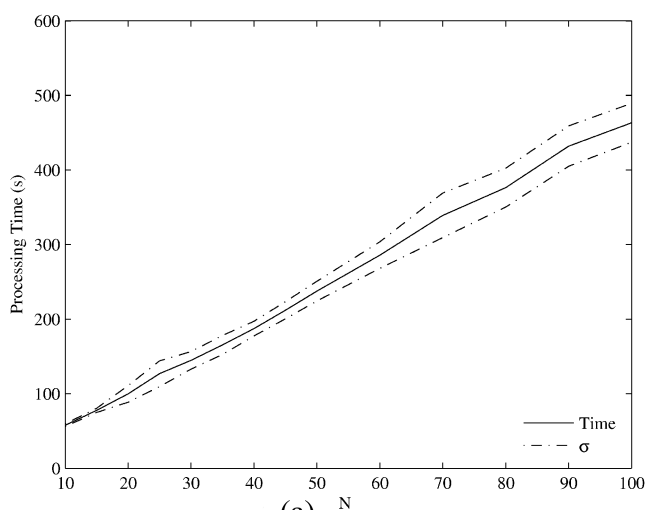

(a)

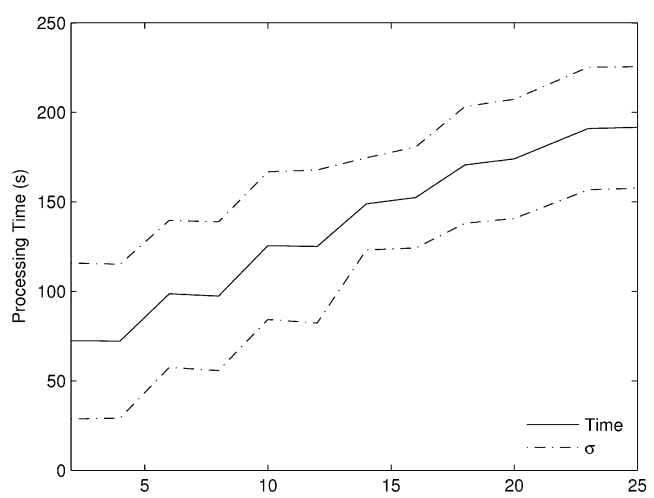

(b) $\mathrm{N}_{\mathrm{f}}$

Figure 2.4: MFBD Processing Time vs $N_{z}$ and $N_{f}$ 
impact on processing time is the number of images required to recover the object. For a set of $K, N \times N$ images, the number of variables increases as $K \times N^{2}$. Previous work indicated that 15 images and 35 Zernike terms would provide a good estimate of the object[33]. Further exploration over a larger data set yielded similar results but additional insight into the estimator's performance. With the number of images set to 50 in order to reduce the influence of the number of images on the outcome, the number of Zernike coefficients was varied from ten to one hundred terms for all three turbulence conditions as shown in Figure 2.5. For all three turbulence cases of $C_{n}^{2}$ additional terms beyond 60 do not significantly improve the MSE.
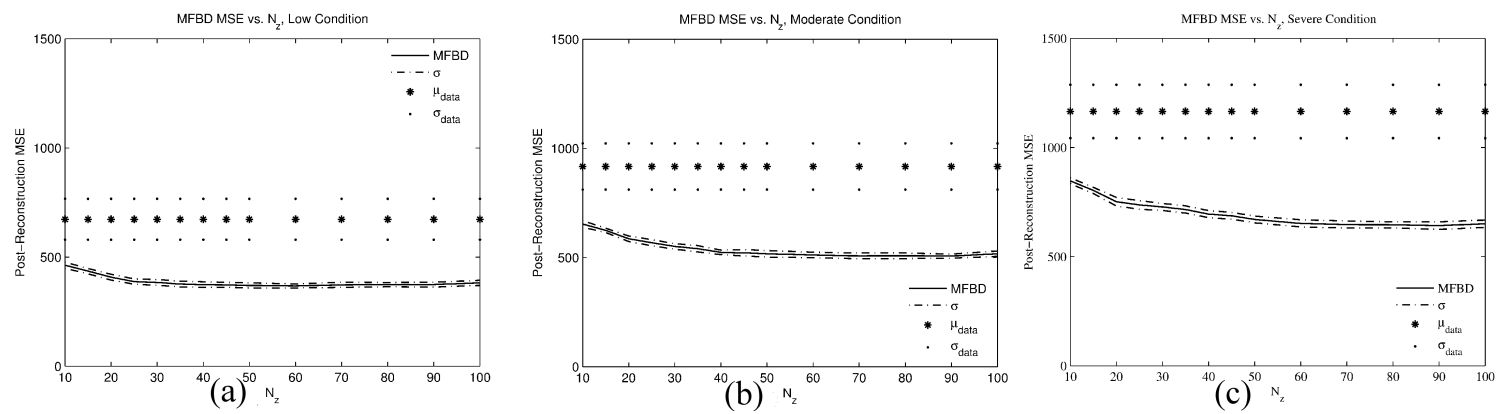

Figure 2.5: Demonstration of the residual post-reconstruction MSE as the number of Zernike coefficients is varied. With the number of frames held constant at 50 the number of Zernike coefficients are varied from 10 to 100 for (a) Low condition $C_{N}^{2}=2.25 \times 10^{-14} m^{(-2 / 3)}$ (b) Moderate condition $C_{N}^{2}=$ $3.75 \times 10^{-14} m^{(-2 / 3)}(\mathrm{c})$ Severe condition $C_{N}^{2}=5.25 \times 10^{-14} m^{(-2 / 3)}$ 


\subsection{Results}

\subsubsection{Gaussian Noise Model}

\subsubsection{Case 1 Low Condition $C_{N}^{2}=2.25 \times 10^{-14} m^{(-2 / 3)}$}

Fig. 2.6 shows that at $N_{f}=2$ and thereafter the estimator can be expected to perform better than the average mean square error for the simulated image which was 673 . Marginal improvement in MSE declines at $N_{f}=12$, reaching a maximum improvement of approximately 40 percent over the average mean square error over the entire data set. However, if processing time is not of consequence, the MSE and its standard deviation continues to improve as additional images are added. Examining the results of the Zernike term evaluation shown in Fig.2.5, 60 Zernike coefficients were used to characterize the PSFs and the results are compared to the estimator's performance using 30 Zernike terms in Fig. 2.6. The use of additional Zernike terms does not add as much processing time as using more input images but each reconstruction will take longer as further Zernike terms are used to characterize the PSFs. Figure 2.7 shows that increasing the number of frames used in the reconstruction will reduce the variance of the estimate but after 15 frames are used, the improvement begins to decline. The incremental improvement in either the estimate or the variance is not worth the additional time consumed. Figure 2.8 shows 
the effect of additive Gaussian noise on the estimators performance. This noise would be characteristic of sensor read-out noise. As expected when more noise is added to the input images the estimator performance declines. The diffraction limited image is compared to a sample of the simulated turbulent image data set images and a sample reconstructed object in Fig. 2.9.

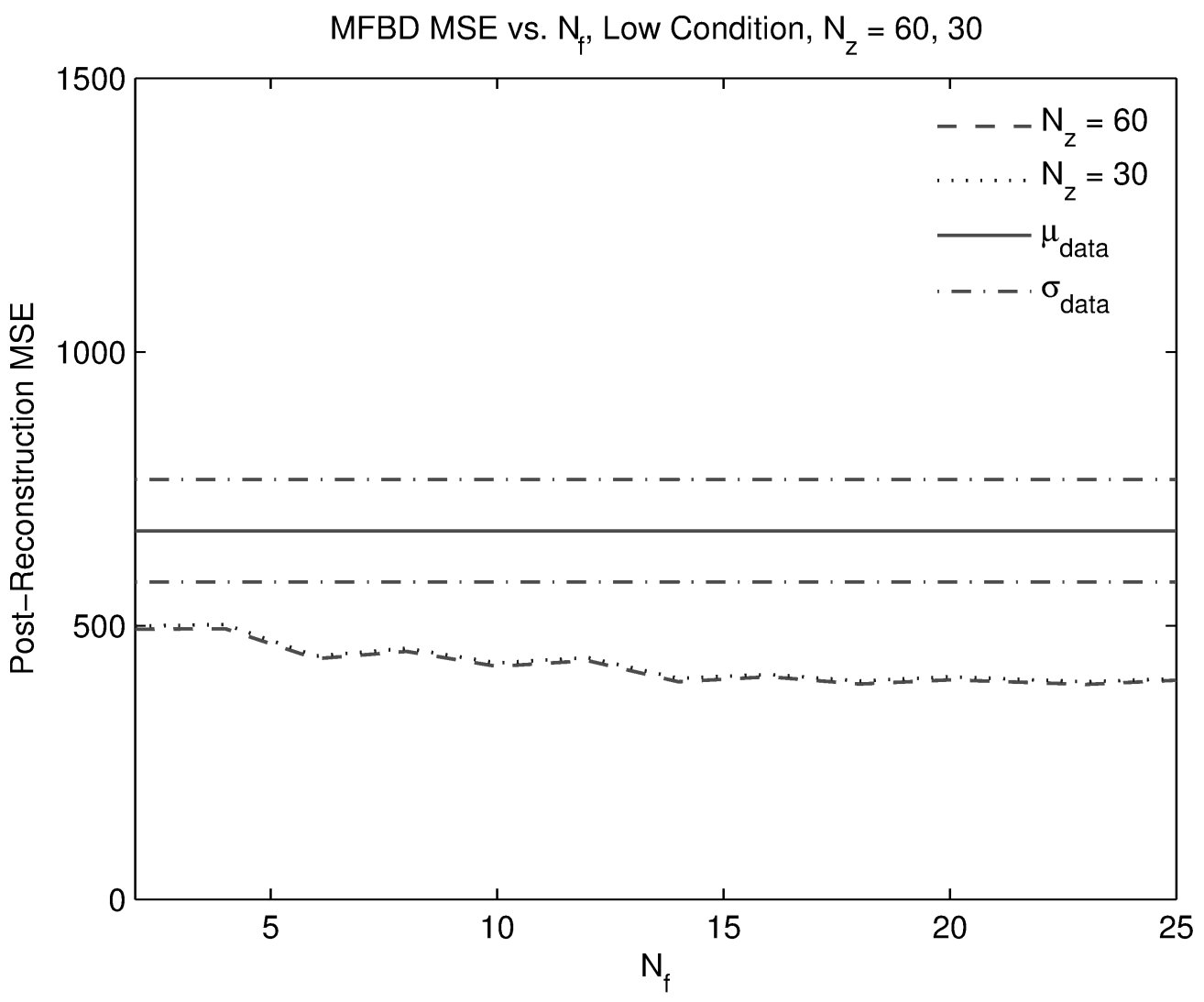

Figure 2.6: Mean Square Error vs. number of frames. Case 1 Low turbulence $C_{N}^{2}=2.25 \times 10^{-14} m^{(-2 / 3)}$ with 30 and 60 Zernike terms 


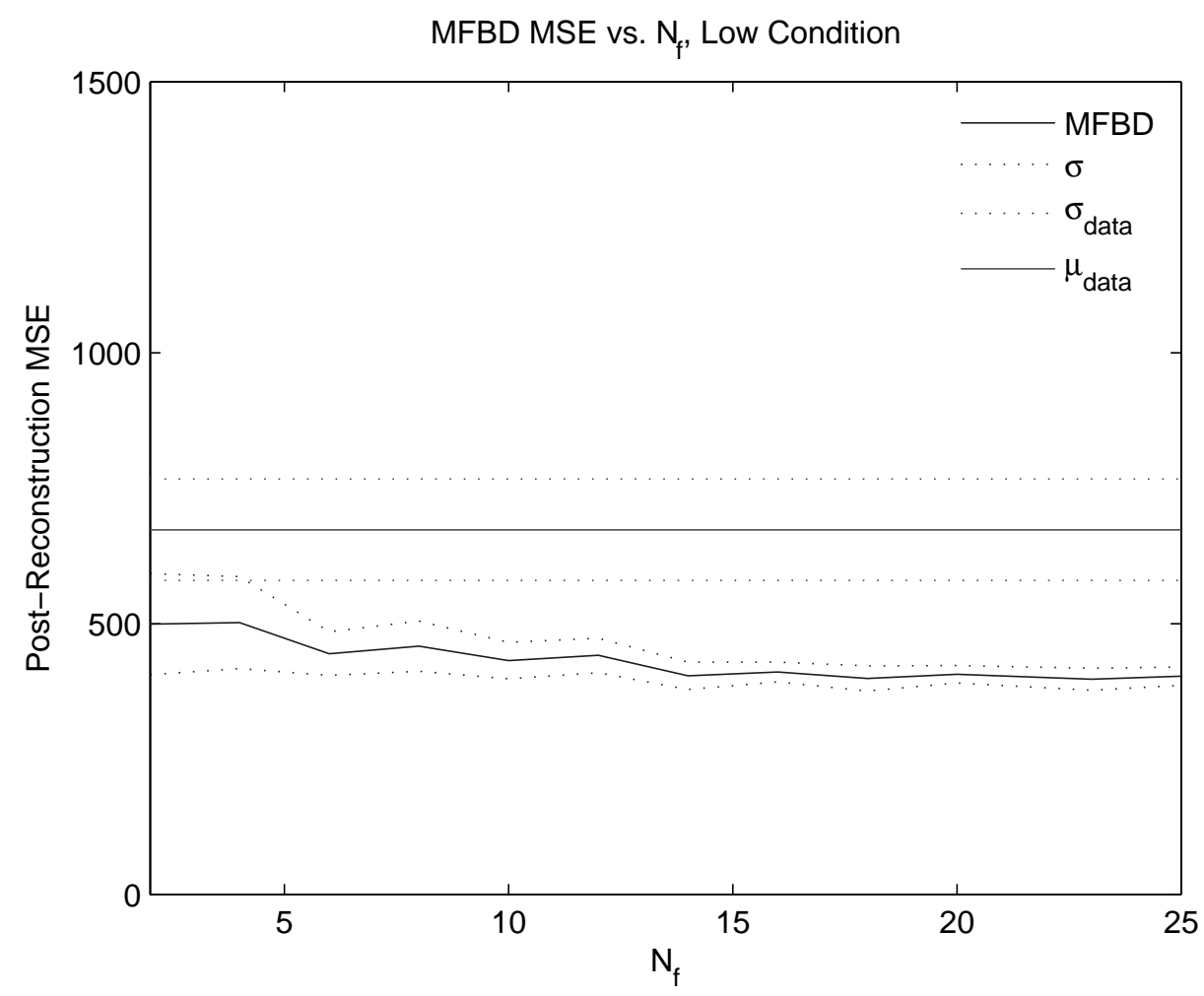

Figure 2.7: Residual reconstruction MSE as the number of frames used in the reconstruction is increased. The solid lines represent the mean value over $\mathrm{N}$ reconstructions, and the mean error over the entire data set. Dashed lines represent the variance of the estimate for a given number of frames used in the reconstruction. Case 1 Low turbulence $C_{N}^{2}=2.25 \times$ $10^{-14} m^{(-2 / 3)} N_{z}=30$

\subsubsection{Case 2 Moderate Condition $C_{N}^{2}=3.75 \times 10^{-14} m^{(-2 / 3)}$}

Figure 2.10 shows (a) the diffraction limited image, (b) a sample recorded image, and (c) a sample reconstructed object. We see in Fig. 2.11 that the MFBD estimator will consistently perform on average better than the average image error as soon as the size of the processing window reaches two frames. At $N_{f}=2$ and thereafter the estimator can 


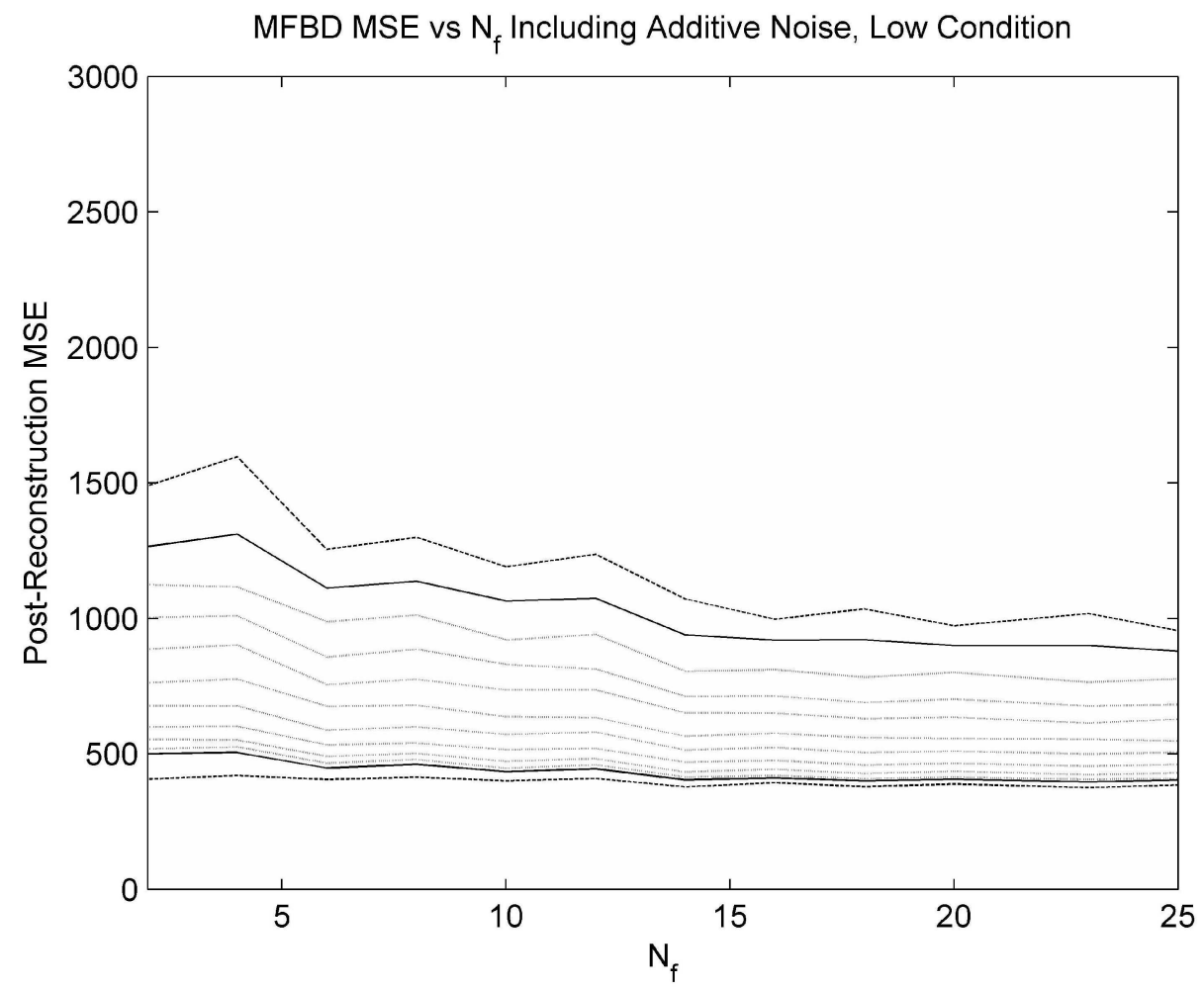

Figure 2.8: Residual reconstruction MSE as the number of frames used in the reconstruction is increased in the presence of additive noise. The solid lines represent the mean reconstruction error for $\sigma_{n}=1$ and $\sigma_{n}=10$. The dotted lines represent the mean performance for $\sigma_{n}=2,3 \ldots 9$. The dashed lines at the limit of the plots represents the variance for the two bounding cases. Case 1 Low turbulence $C_{N}^{2}=2.25 \times 10^{-14} m^{(-2 / 3)} N_{z}=30$.

be expected to perform better than the average mean square error of the simulated image.

The improvement in MSE available by including additional input frames hits a maximum of approximately 25 percent of full scale at $N_{f}=14$. Neither the MSE nor the standard deviation improves significantly as additional input images are added to the stack. Informed by the results of the Zernike term sweeps discussed above, the estimator was run using 30 Zernike coefficients to characterize the PSFs. The results are compared in Figure. 2.11, Figure 2.12 shows that increasing the number of frames used in the reconstruction will 


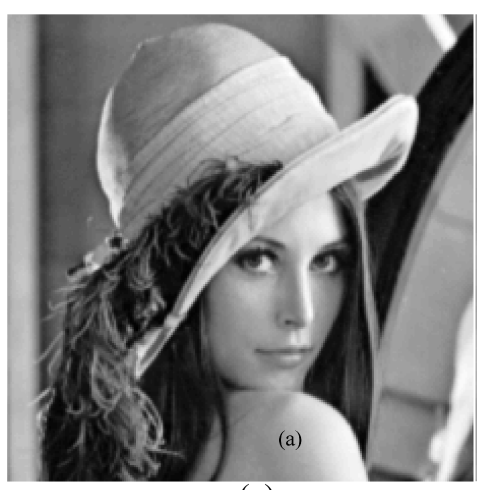

(a)

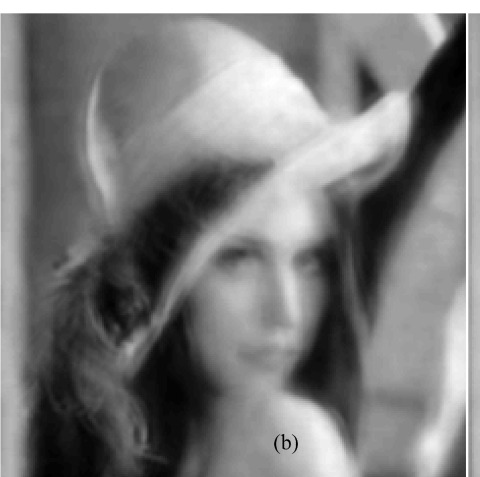

(b)

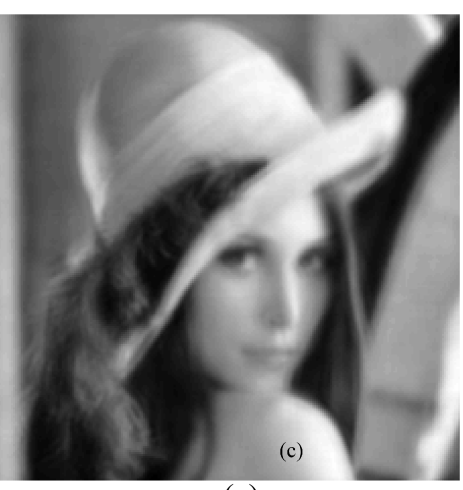

(c)

Figure 2.9: Case 1 sample images. Compares the (a) diffraction-limited image with (b) the single sample image and (c) a sample reconstructed object.

reduce the variance of the estimate but after 15 frames are used, the improvement begins to decline. The incremental improvement in either the estimate or the variance is not worth the additional time consumed. Figure 2.13 shows the effect of additive Gaussian noise on the estimators performance. This noise would be characteristic of sensor read-out noise. As expected, when more noise is added to the input images the estimator performance declines. The use of additional Zernike terms does not incur as large a computational penalty as that associated with adding additional frames but each reconstruction will take longer. The incremental improvement in mean square error is not worth the additional processing time. 


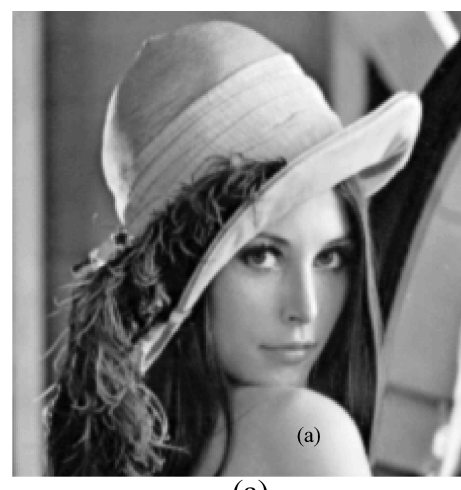

(a)

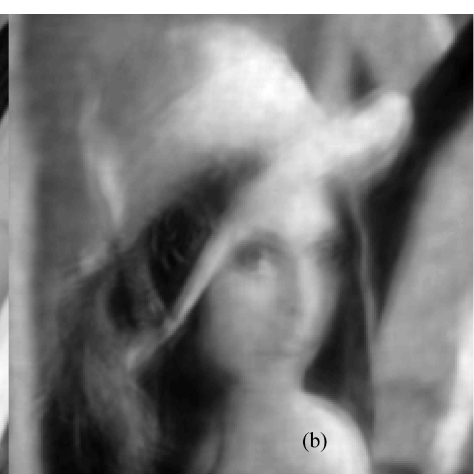

(b)

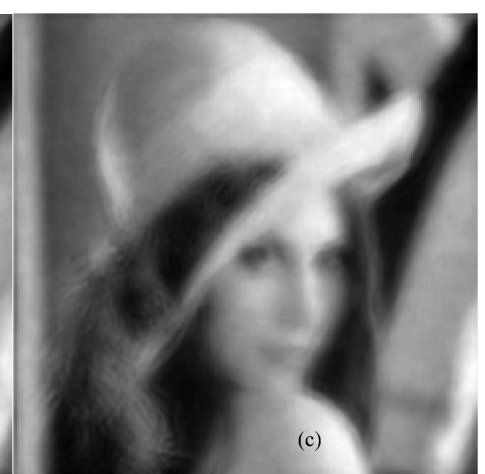

(c)

Figure 2.10: Case 2 sample images. Compares the (a) diffraction-limited image with (b) the single sample image and (c) a sample reconstructed object.

\subsubsection{Case 3 Severe Condition $C_{N}^{2}=5.25 \times 10^{-14} m^{(-2 / 3)}$}

Again we see in Fig. 2.15 that the estimator requires at least two input frames to reliably produce an estimate of the object that has a lower mean square error than 1165 , the average simulated image's MSE. At $N_{f}=2$ and thereafter the estimator can be expected to perform better than the average mean square error of the recorded image. The improvement in MSE available by including additional input frames hits a maximum of approximately 36 percent of full scale at $N_{f}=14$ and neither the MSE nor the standard deviation improves significantly as additional input images are added to the processing stack. Figure 2.14 shows (a) the diffraction limited image, (b) a sample recorded image, and (c) a sample reconstructed object. Informed by the results of the Zernike term sweeps discussed above, the estimator was run using 30 Zernike coefficients to characterize the PSFs. The results are compared in Figure. 2.15. The use of additional Zernike terms does not incur as 


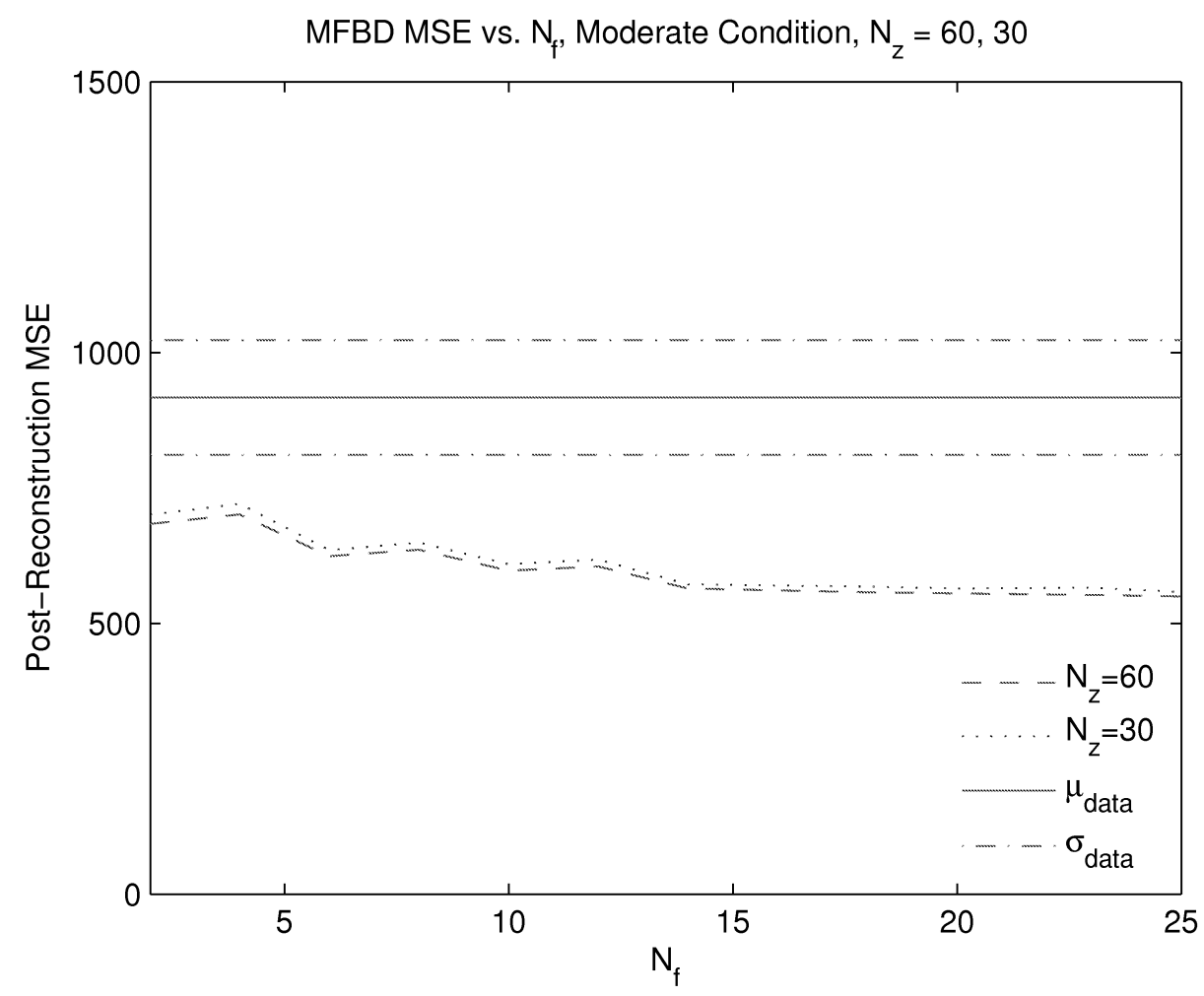

Figure 2.11: Mean Square Error vs. number of frames. Case 2 Moderate turbulence $C_{N}^{2}=3.75 \times 10^{-14} m^{(-2 / 3)}$ with 30 and 60 Zernike terms

large a computational penalty as that associated with adding additional frames but each reconstruction will take longer. Figure 2.16 shows that increasing the number of frames used in the reconstruction will reduce the variance of the estimate but after 15 frames are used, the improvement begins to decline. The incremental improvement in either the estimate or the variance is not worth the additional time consumed. Figure 2.17 shows the effect of additive Gaussian noise on the estimators performance. This noise would be characteristic of sensor read-out noise. As expected when more noise is added to the input images the estimator performance declines. As shown, the incremental improvement in mean square error is not worth the additional processing time. 


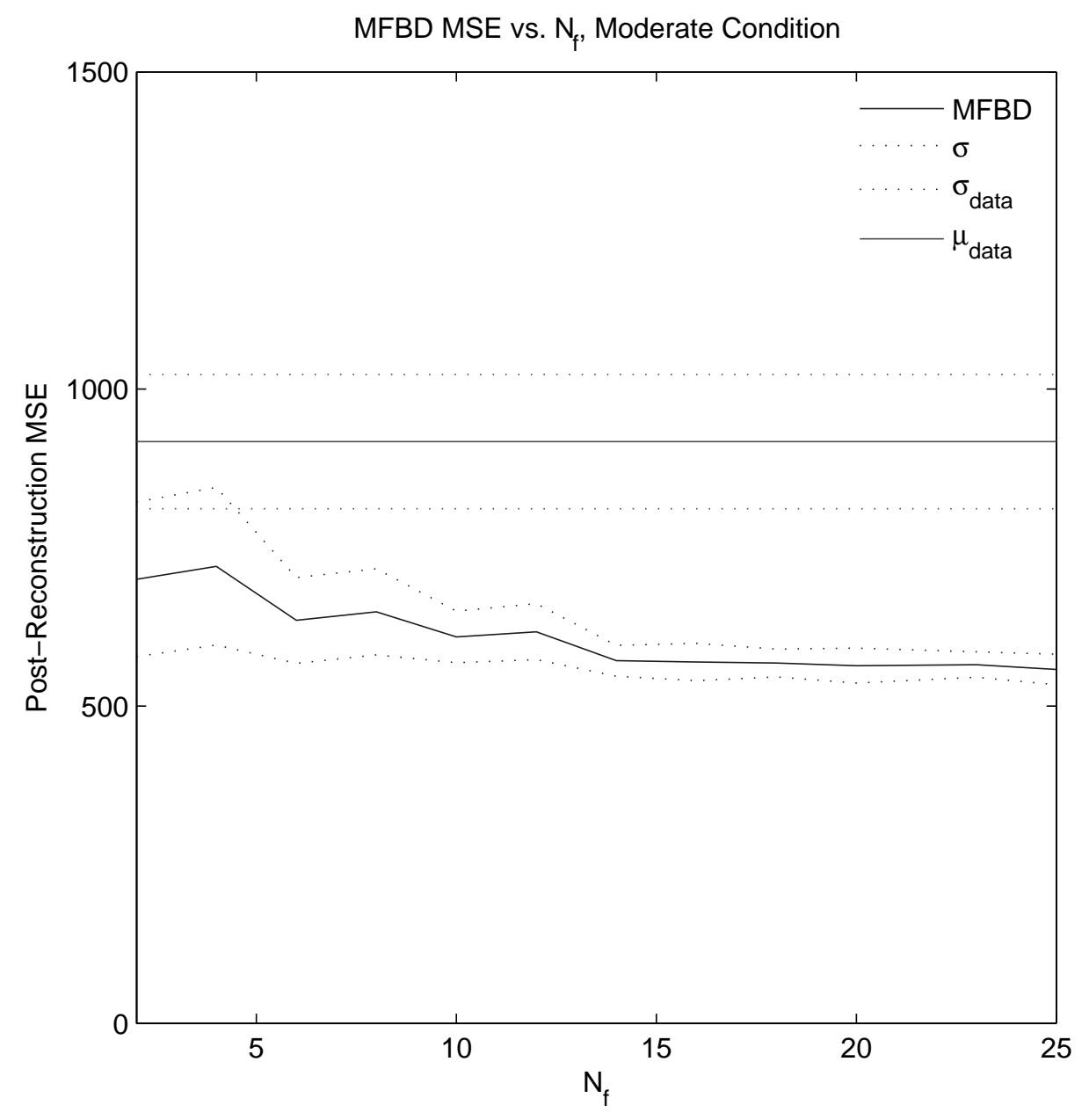

Figure 2.12: Residual reconstruction MSE as the number of frames used in the reconstruction is increased. The solid lines represent the mean value over $\mathrm{N}$ reconstructions, and the mean error over the entire data set. Dashed lines represent the variance of the estimate for a given number of frames used in the reconstruction. Case 2 Moderate turbulence $C_{N}^{2}=$ $3.75 \times 10^{-14} m^{(-2 / 3)}, N_{z}=30$ 


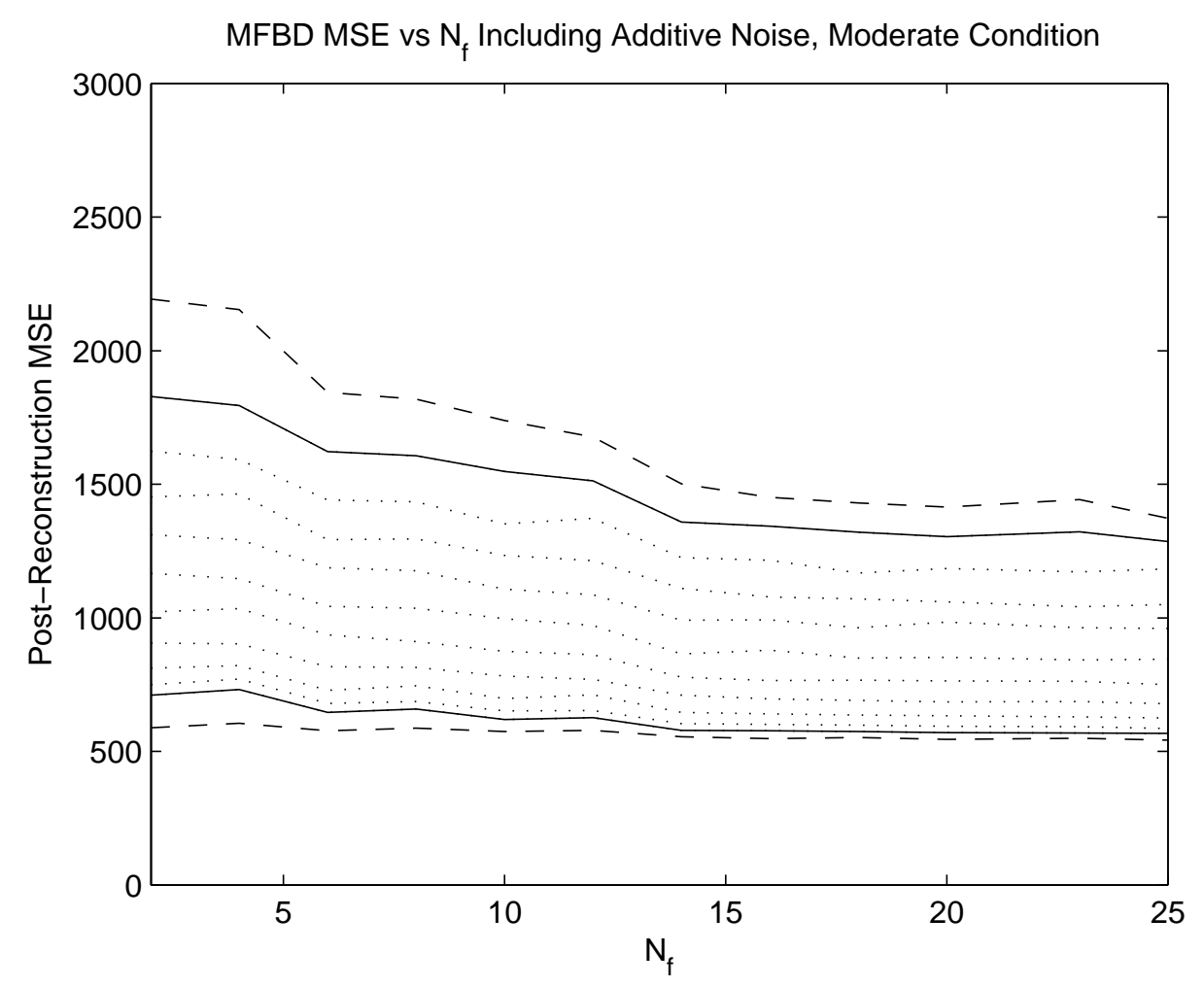

Figure 2.13: Residual reconstruction MSE as the number of frames used in the reconstruction is increased in the presence of additive noise. The solid lines represent the mean reconstruction error for $\sigma_{n}=1$ and $\sigma_{n}=10$. The dotted lines represent the mean performance for $\sigma_{n}=2,3 \ldots 9$. The dashed lines at the limit of the plots represents the variance for the two bounding cases. Case 2 Moderate turbulence $C_{N}^{2}=3.75 \times 10^{-14} m^{(-2 / 3)} N_{z}=30$

\subsubsection{Poisson Noise Model Mean Photon Rate $2 \times 10^{6}, 30$ photons per pixel}

Each set of 1000 turbulent images, representing the three turbulence cases was used to generate a set of speckle images with a mean photon count per image of $2 \times 10^{6}$. Each set of images was processed using the MFBD methods described above using the cost function 


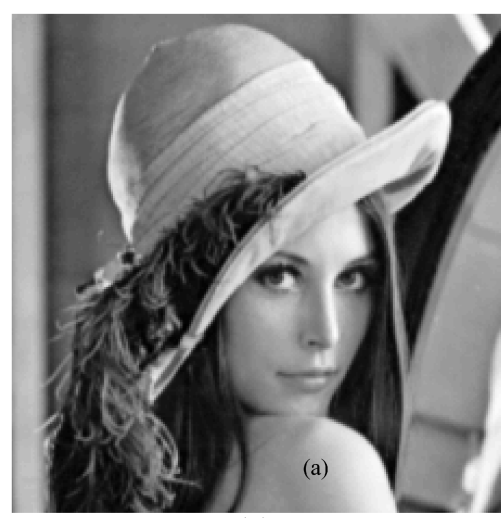

(a)

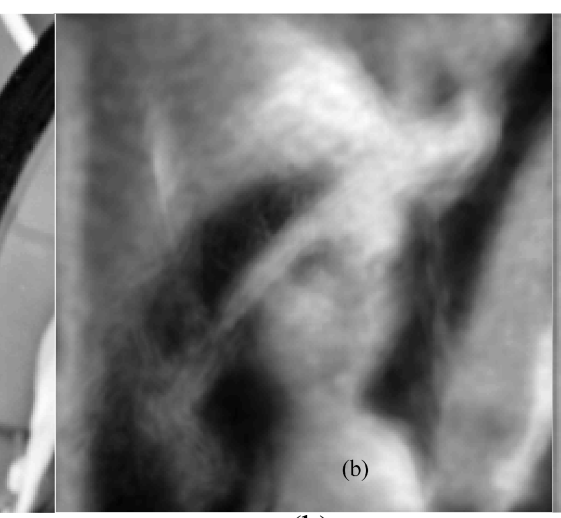

(b)

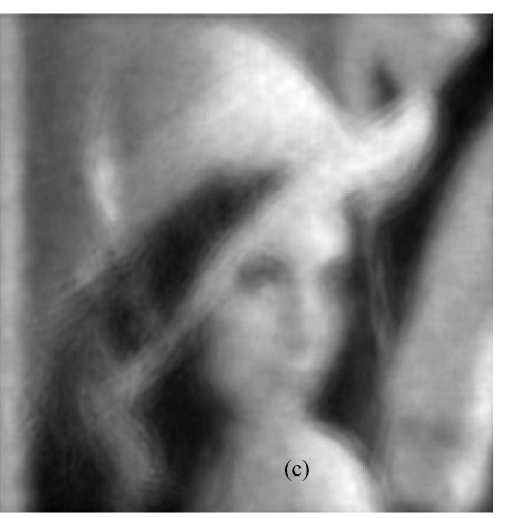

(c)

Figure 2.14: Case 3 sample images. Compares the (a) diffraction-limited image with (b) the single sample image and (c) a sample reconstructed object.

and gradient described in equations $2.22,2.23$, and 2.24

\subsubsection{Case 1 Low Condition $C_{N}^{2}=2.25 \times 10^{-14} m^{(-2 / 3)}$}

Examining Fig. 2.19 we see that on average, MFBD performance is less than the input images until 50 input frames are used in each reconstruction. At $N_{f}=50$ and thereafter the estimator can be expected to produce an estimate that has a value lower than 2095 , the average mean square error of the images in the simulated data set. Marginal improvement in MSE continues to improve as additional frames are added to the image stack reaching a maximum of about 38 percent improvement over the average MSE across the data set. However, if processing time is not of consequence, the MSE and its standard deviation continues to improve as additional images are added to the stack of images presented to the estimator, so additional improvement in the quality of the image is available. The 


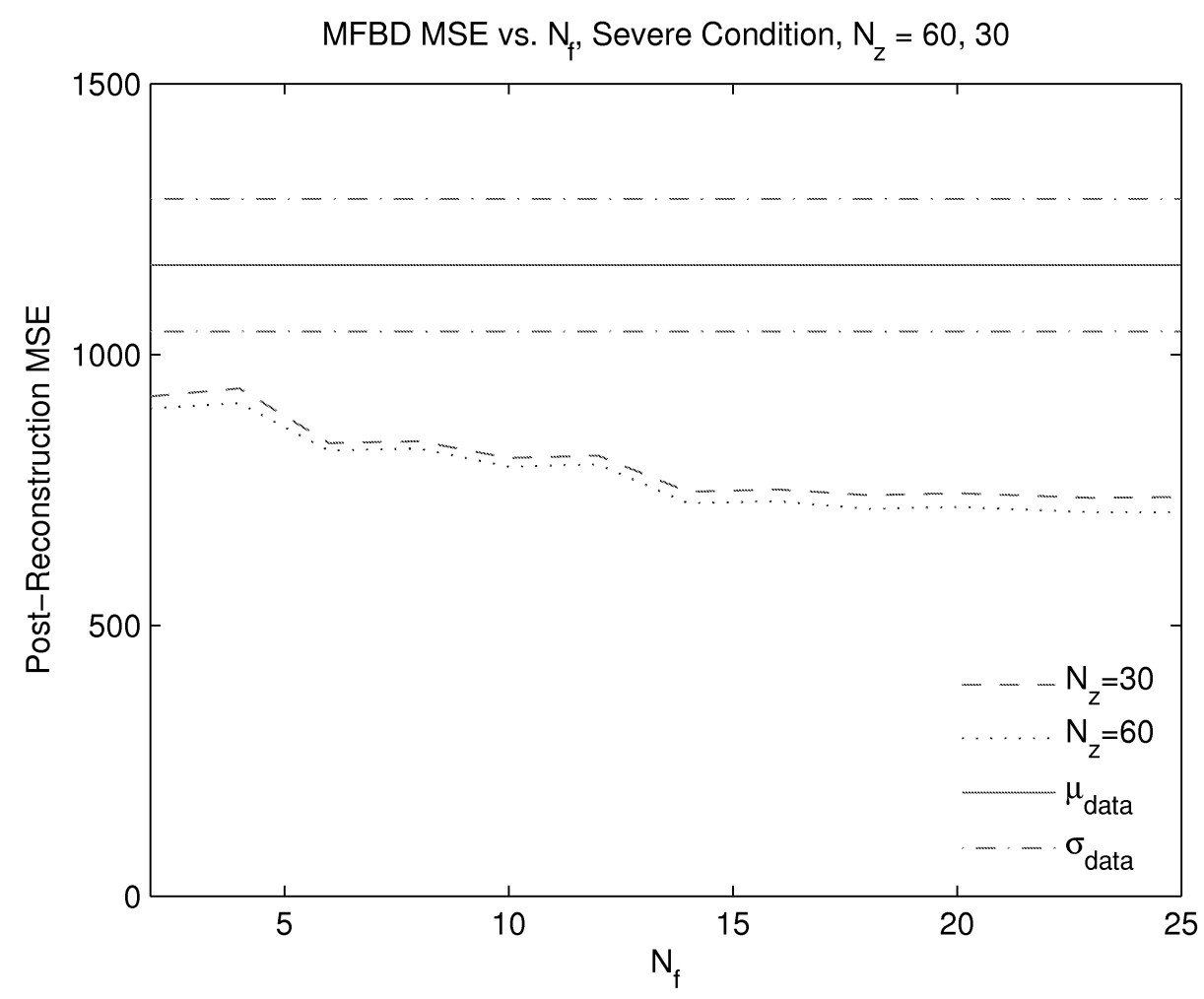

Figure 2.15: Mean Square Error vs. number of frames. Case 3 Severe turbulence $C_{N}^{2}=5.25 \times 10^{-14} m^{(-2 / 3)}$ with 30 and 60 Zernike terms

diffraction limited image is compared to a sample simulated turbulent image and a sample reconstructed object as shown in Fig. 2.18,

\subsubsection{Case 2 Moderate Condition $C_{N}^{2}=3.75 \times 10^{-14} m^{(-2 / 3)}$}

Figure 2.20 shows (a) the diffraction limited image, (b) a sample recorded image, and (c) a sample reconstructed object using 175 frames to estimate the object. Again we see in Fig. 2.21 that the MFBD estimator will not perform on average any better than 2285, the average simulated turbulent image error until the number of images processed reaches 50 frames. At $N_{f}=50$ and thereafter the estimator can be expected to perform better than the 


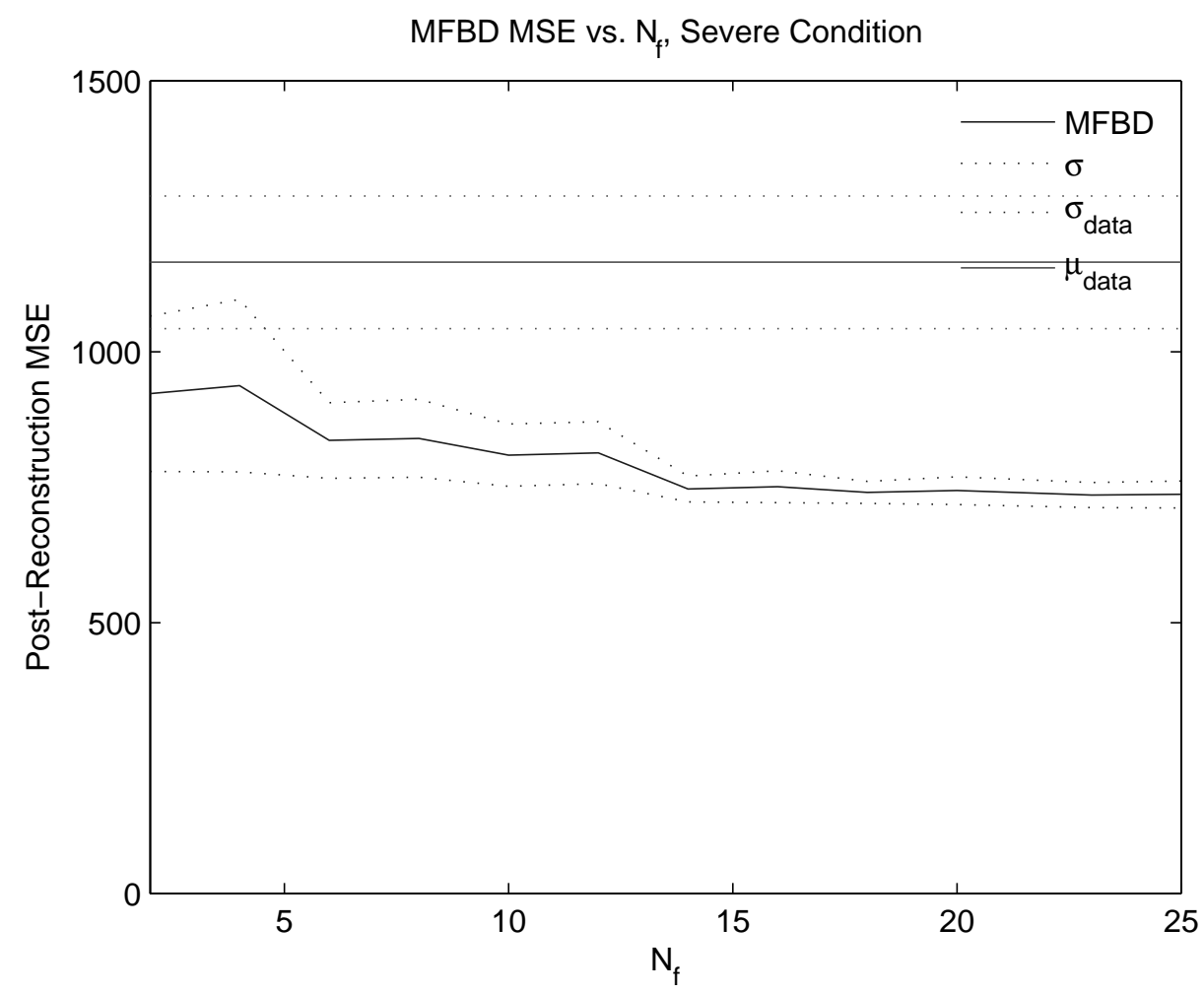

Figure 2.16: Residual reconstruction MSE as the number of frames used in the reconstruction is increased. The solid lines represent the mean value over $\mathrm{N}$ reconstructions, and the mean error over the entire data set. Dashed lines represent the variance of the estimate for a given number of frames used in the reconstruction. Case 3 Severe turbulence $C_{N}^{2}=$ $5.25 \times 10^{-14} m^{(-2 / 3)} N_{z}=30$

average mean square error of the simulated image reaching a maximum of about 34 percent improvement. The marginal improvement in MSE available by including additional input frames begins to decline at about $N_{f}=175$ and neither the MSE nor the standard deviation improves significantly as additional input images are processed. 


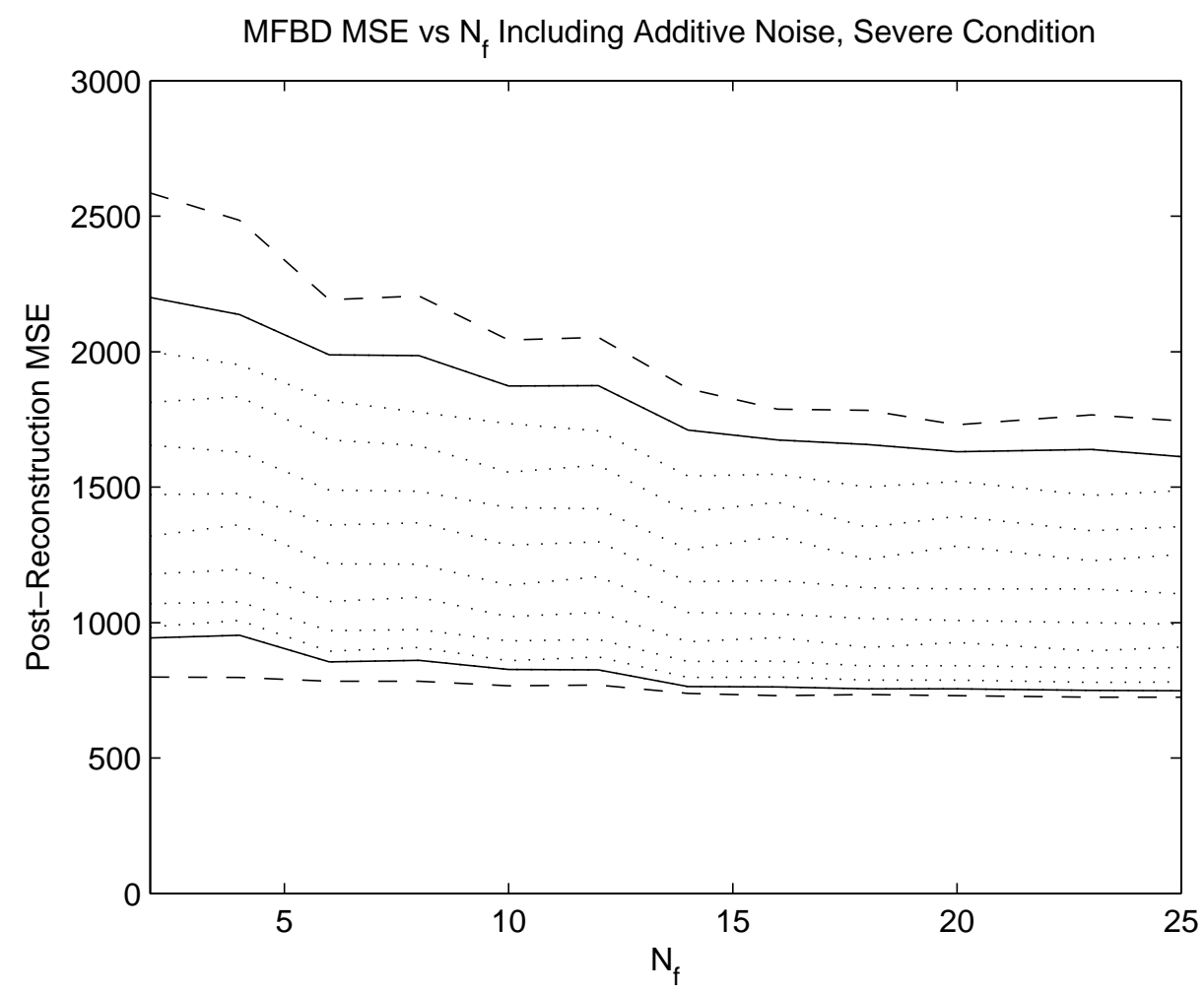

Figure 2.17: Residual reconstruction MSE as the number of frames used in the reconstruction is increased in the presence of additive noise. The solid lines represent the mean reconstruction error for $\sigma_{n}=1$ and $\sigma_{n}=10$. The dotted lines represent the mean performance for $\sigma_{n}=2,3 \ldots 9$. The dashed lines at the limit of the plots represents the variance for the two bounding cases. Case 3 Severe turbulence $C_{N}^{2}=5.25 \times 10^{-14} m^{(-2 / 3)}$ with $N_{z}=30$

\subsubsection{Case 3 Severe Condition $C_{N}^{2}=5.25 \times 10^{-14} m^{(-2 / 3)}$}

Again we see in Fig. 2.23 that the MFBD estimator will not perform on average any better than the average image error until the number of images offered to the estimator reaches 50 frames. At $N_{f}=50$ and thereafter the estimator can be expected to perform better than the average mean square error(2456)of the simulated image reaching a maximum of about 33 percent. The marginal improvement in MSE available by including additional input 


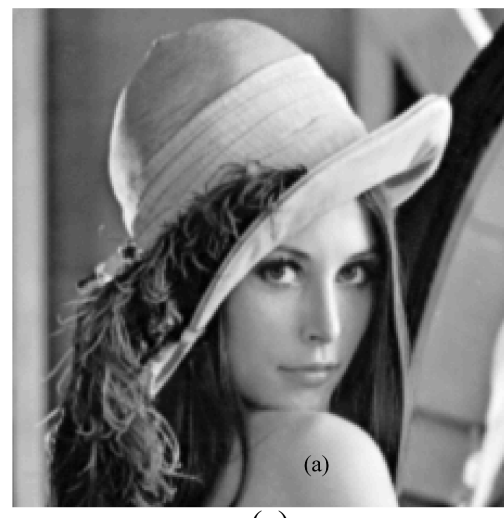

(a)

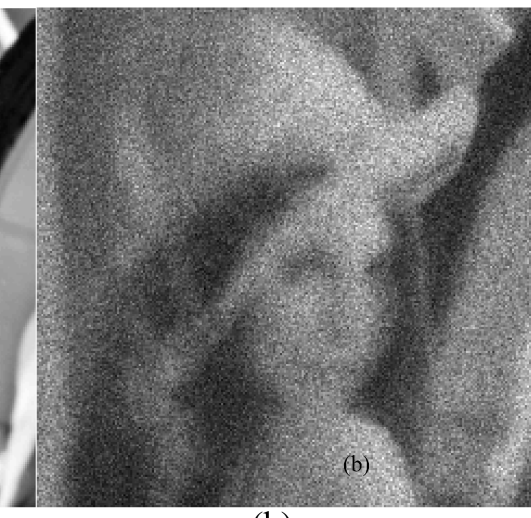

(b)

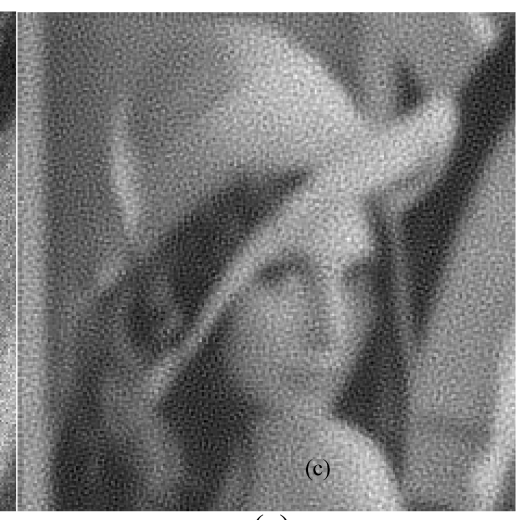

(c)

Figure 2.18: Case 1 sample images, Mean Photon Rate $2 \times 10^{6}, C_{N}^{2}=2.25 \times$ $10^{-14} m^{(-2 / 3)}$. Compares the (a) diffraction-limited image with (b) a single sample image and (c) a sample reconstructed object.

frames begins to decline at about $N_{f}=175$ and neither the MSE nor the standard deviation seems to improve significantly from there as additional input images are added to the stack. Figure 2.22 shows (a) the diffraction limited image, (b) a sample recorded image, and (c) a sample reconstructed object.

\subsection{Conclusions}

The performance of an unconstrained optimization-based multi-frame blind deconvolution estimator was evaluated in terms of the mean squared error between the reconstructed object and a diffraction limited image. Three 1000-image data sets of a single image distorted by low, moderate, and severe turbulence were generated using a horizontal imaging simulator that includes anisoplanatic effects. The data sets were then applied to the estimator and its MSE performance evaluated. If a hardware implementation were to 


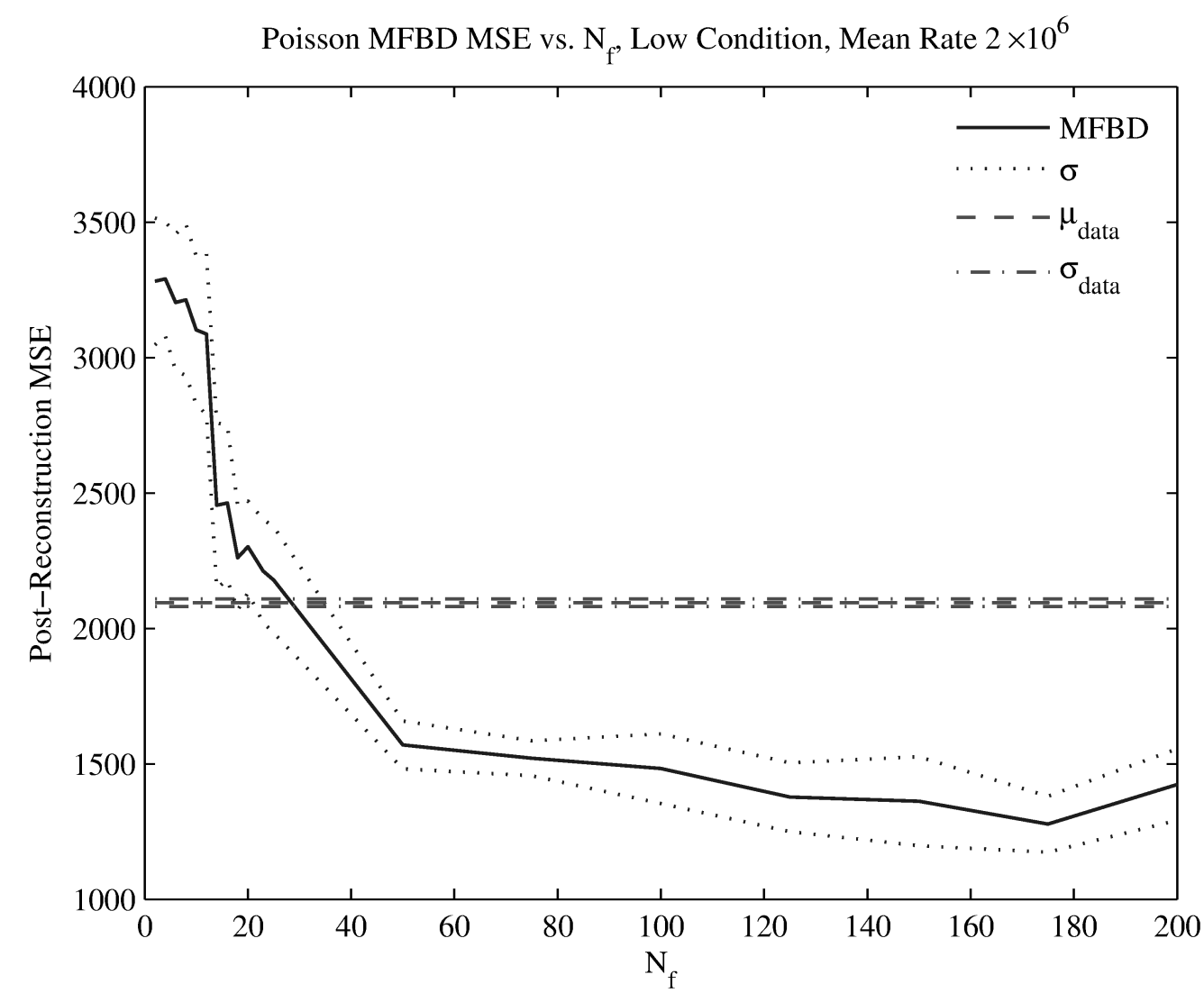

Figure 2.19: Mean Square Error vs. number of frames. Poisson Case 1 Low turbulence $C_{N}^{2}=2.25 \times 10^{-14} m^{(-2 / 3)}$, mean photon rate $2 \times 10^{6}$

be produced with a fixed, or limited set of operator options, a wide variety of turbulence cases would be well served by a selection of 14 images and 30 polynomial terms for use with the estimator. Point performance estimates, using a data set of 1000 simulated turbulence corrupted images, indicate that the algorithm is capable of producing 40,25 and 36 percent improvements in MSE for low, moderate, and severe-anisoplanitic turbulence cases respectively under the assumption that the phase errors can be characterized as a Gaussian distribution. For all simulated turbulence cases, significant reductions were observed with as few as two input images. For the Poisson case, significant results were achieved with as few as 50 frames, but 175 frames would be a reasonable place to design a 


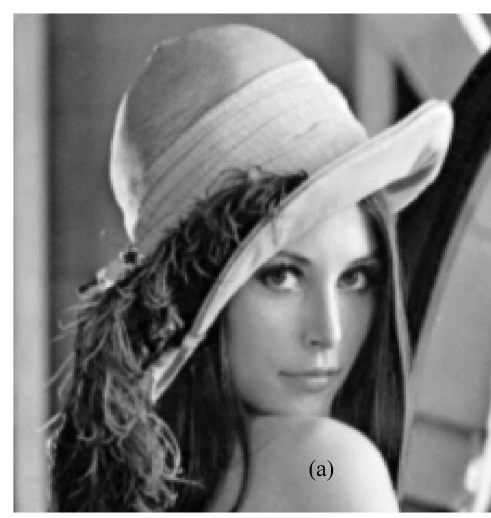

(a)

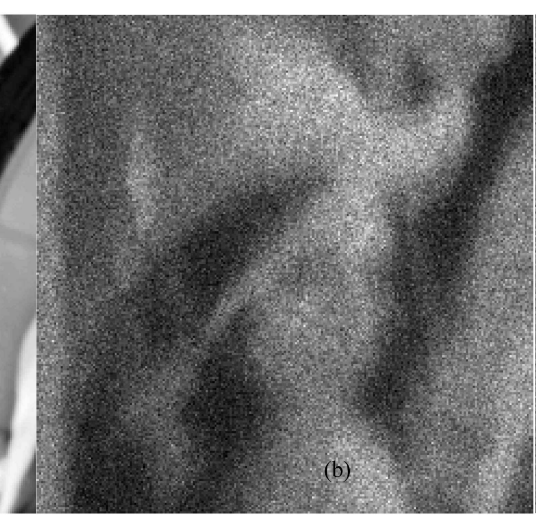

(b)

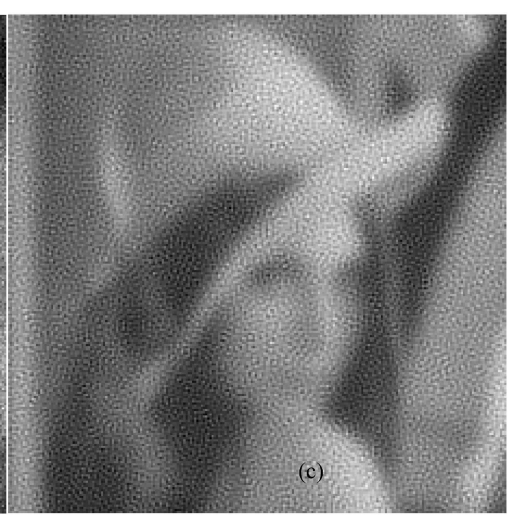

(c)

Figure 2.20: Case 2 sample images, Mean Photon Rate $2 \times 10^{6}, C_{N}^{2}=$ $3.75 \times 10^{-14} m^{(-2 / 3)}$. Compares the (a) diffraction-limited image with (b) a single sample image and (c) a sample reconstructed object.

system that would be able to cope with a variety of atmospheric turbulence and light levels.

Chapter 5 explores the possibility of speeding up the reconstruction by providing a better initial guess at the object. Simulated annealing techniques could also be used to perturb the estimate away from a local minimum, and may prove to be an effective answer to local minimum trapping. 


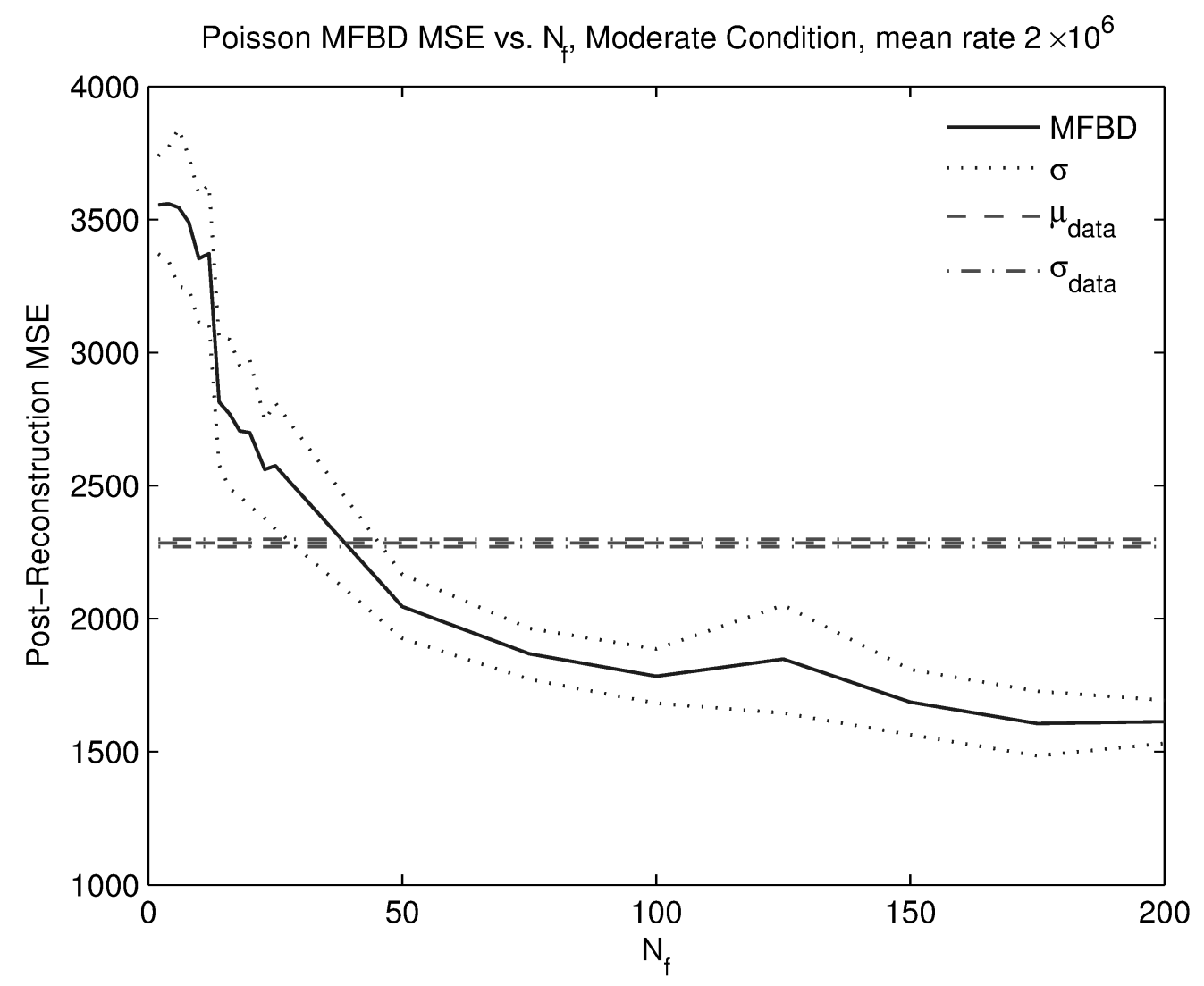

Figure 2.21: Mean Square Error vs. number of frames. Poisson Case 2 Moderate turbulence $C_{N}^{2}=3.75 \times 10^{-14} m^{(-2 / 3)}$, mean photon rate $2 \times 10^{6}$

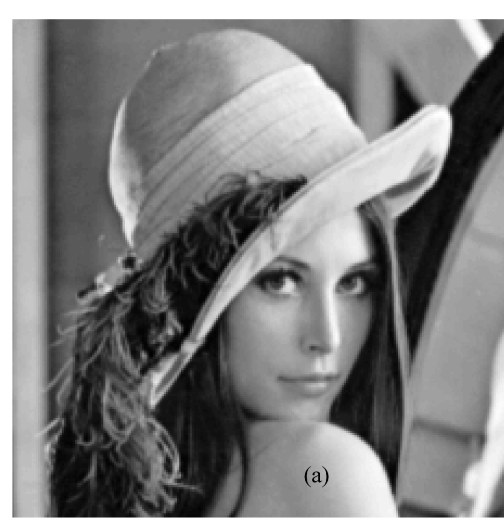

(a)

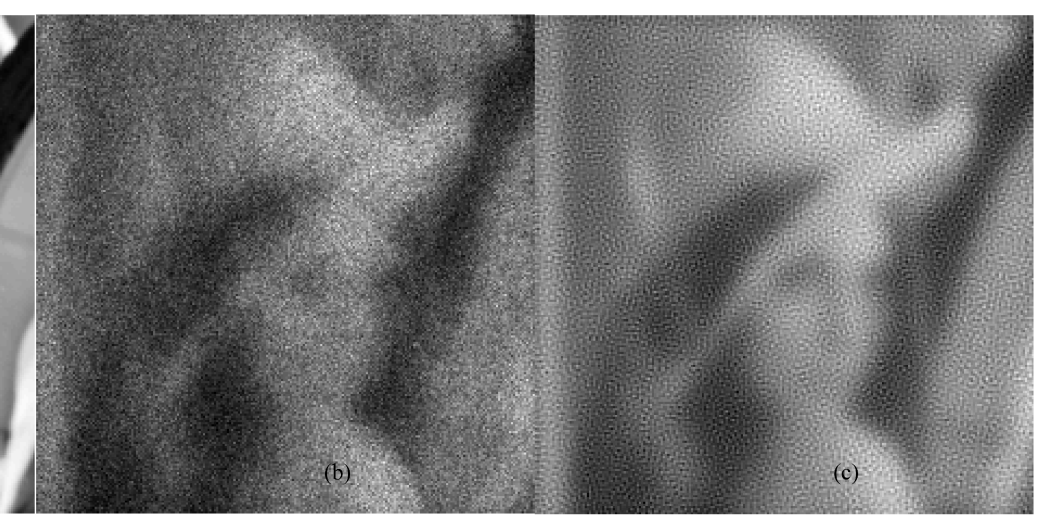

(b) (c)

Figure 2.22: Case 3 sample images, Mean Photon Rate $2 \times 10^{6}, C_{N}^{2}=$ $5.25 \times 10^{-14} m^{(-2 / 3)}$. Compares the (a) diffraction-limited image with (b) a single sample image and (c) a sample reconstructed object. 


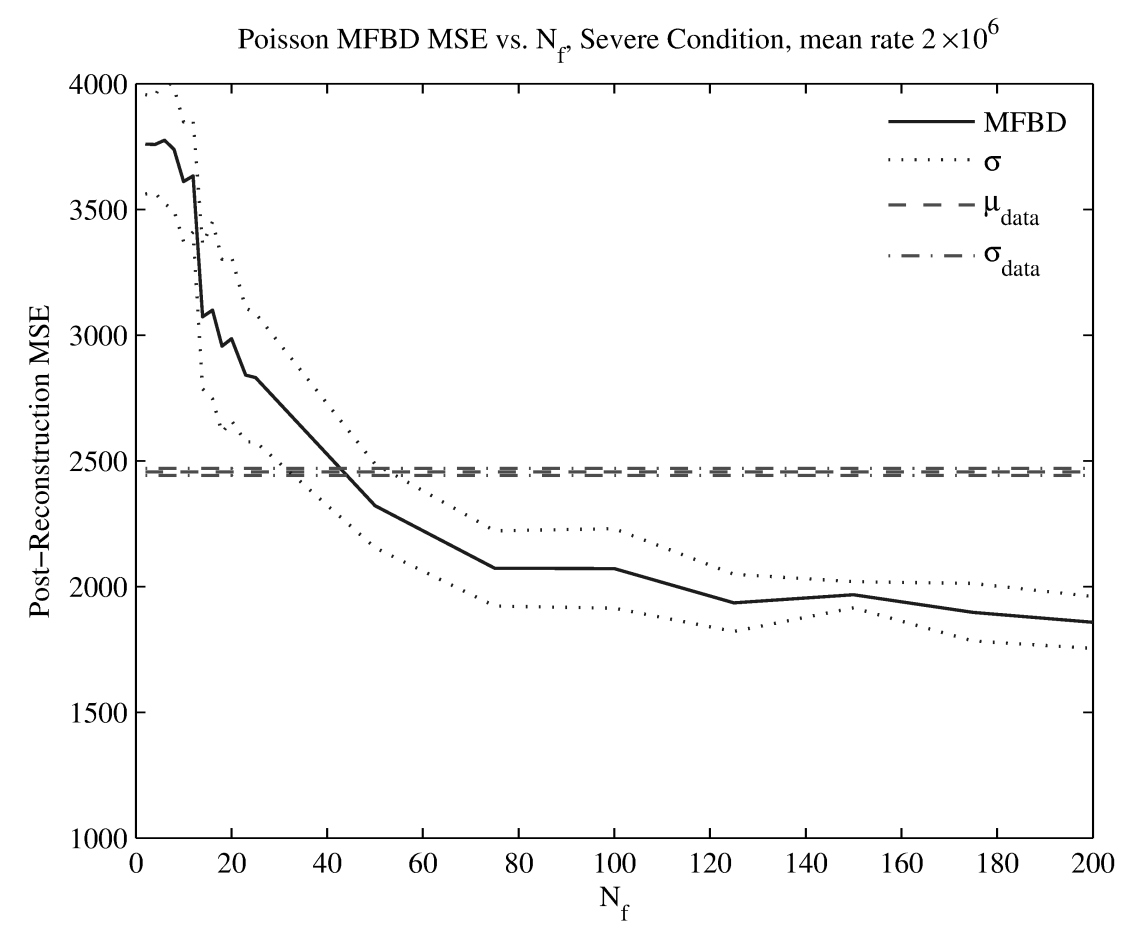

Figure 2.23: Mean Square Error vs. number of frames. Poisson Case 3 Severe turbulence $C_{N}^{2}=2.25 \times 10^{-14} m^{(-2 / 3)}$, mean photon rate $2 \times 10^{6}$ 


\section{Chapter 3}

A Comparison of the MSE Performance

of Speckle and MFBD Image

\section{Reconstruction Techniques}

${ }^{1}$ The material in this chapter has been accepted for publication in Optical Engineering. 


\subsection{Introduction}

This chapter compares the performance of speckle imaging techniques and multi-frame blind deconvolution at estimating object intensities from simulated anisoplanatic images that have been corrupted by atmospheric turbulence using the number of input frames and scene illumination levels as the operational parameters. Using a horizontal imaging simulation model [23] three sets of 1000 simulated, turbulent images based on an image common in the literature were created. In order to explore the techniques performance under multiple illumination conditions, each set of simulated images, representing the the three turbulence cases was used to generate a set of speckle images with a mean photon count per image of $2 \times 10^{6}, 1 \times 10^{6}$, and $5 \times 10^{5}$. The effectiveness of each technique is assessed by calculating the MSE between the resulting recovered object and the diffraction limited image.

I find that the both speckle-imaging and MFBD reconstructed objects show significant improvement in mean squared error compared to the average mean square error between all the images in a data set and the associated diffraction limited image. Speckle-imaging techniques reduce the MSE 46, 42 and 47 percent on average for low, moderate, and severe cases, respectively using 15 input frames under daytime conditions. Similarly, the MFBD method provides, 40, 25, and 36 percent improvements in MSE on average under the same conditions. Under low light conditions (on average fewer than 100 photons per pixel), 
improvements of 39, 29 and 27 percent are available using speckle imaging methods and 25 input frames and 38, 34 and 33 respectively for the MFBD method and 150 input frames.

The remainder of this chapter is organized as follows. In Section 3.2, I discuss horizontal imaging as it applies to the reconstruction of turbulent images under anisoplanatic conditions and develop foundations of the bispectrum and the objective functions to be applied to the L-BFGS optimization routines. In Section 3.3 the object recovery methods for speckle imaging techniques and MFBD are described for both Gaussian and Poisson noise models. In section 3.4 the comparative results of processing for bispectrum and MFBD are presented under all three turbulence cases and, in the case of the Poisson noise model, for three turbulence conditions and three diminishing values of mean photon counts. Finally in section 3.5 summative conclusions regarding the relative merits of these two strategies are presented.

\subsection{Background}

\subsubsection{Horizontal Imaging}

I begin my discussion by examining some assumptions regarding the horizontal imaging problem. The resolution available to an imaging system is limited by atmospheric turbulence. The atmospheric coherence radius, $r_{0}$, is commonly used to define the resolving 
power of an imaging system in terms of an equivalent diffraction limited system's radius. $r_{0}$ is defined by Fried for spherical wave propagation[21][18] as

$$
r_{0}=0.185\left[\frac{4 \pi^{2}}{k^{2} \int_{0}^{L}\left(\frac{L-z}{L}\right)^{5 / 3} C_{n}^{2}(z) d z}\right]^{3 / 5}
$$

where $\lambda$ is the mean wavelength, the wave number $k=\frac{2 \pi}{\lambda}$, and $\Delta z$ is the distance from the scene to the imaging aperture. The limits of the integral are from $z=0$ at the pupil plane to the scene at $z=L$. For purposes of this study it is assumed that in Equation (3.1) that the refractive-index structure constant $C_{n}^{2}$ is constant over paths that are horizontal or at shallow angles from the aperture [19]. It is further assumed that the strength of the turbulence is such that scintillation effects can be neglected. The data sets simulated for this work can be categorized by the value of $C_{n}^{2}$ : for the low turbulence condition $C_{n}^{2}=2.25 \times 10^{-14} m^{(-2 / 3)}$, for the moderate condition $C_{n}^{2}=3.75 \times 10^{-14} m^{(-2 / 3)}$ and for the severe condition $C_{n}^{2}=5.25 \times 10^{-14} \mathrm{~m}^{(-2 / 3)}$. A complete description of the simulator used to create these data sets is available in [23].

Using Fried's [22] definition for the isoplanatic angle, $\theta_{o}$,

$$
\theta_{o}=\left(2.91 k^{2} C_{n}^{2} \Delta z^{(5 / 3)}\right)^{(-3 / 5)}
$$

and evaluating for the conditions under consideration, the isoplanatic angle is determined to be between 6.6 and $10.8 \mu \mathrm{rad}$. For the conditions simulated here, a single pixel 
Table 3.1

Atmospheric Simulation Turbulence Conditions

\begin{tabular}{|r|c|c|c|c|}
\hline \multicolumn{5}{|c|}{ Atmospheric Turbulence Parameters } \\
\hline $\begin{array}{r}\text { Turbulence } \\
\text { condition }\end{array}$ & $\begin{array}{c}C_{N}^{2} \\
\left(m^{(-2 / 3)}\right)\end{array}$ & $\begin{array}{c}r_{0} \text { spherical case } \\
(\mathrm{cm})\end{array}$ & $\begin{array}{c}\theta_{0} \\
(\mu \text { radians })\end{array}$ & $\begin{array}{c}\theta_{0} \\
(\text { pixels })\end{array}$ \\
\hline Low & $2.25 \times 10^{-14}$ & 3.33 & 10.8 & 4 \\
\hline Moderate & $3.75 \times 10^{-14}$ & 2.45 & 7.75 & 3 \\
\hline Severe & $5.25 \times 10^{-14}$ & 2.01 & 6.63 & 2 \\
\hline
\end{tabular}

in the simulated imaging system captures $2.79 \mu$ radians. Expressing the $\theta_{0}$ values for the low, medium and severe turbulence conditions of the simulation, it can be seen that the isoplanatic patch covers 4,3 and 2 pixels in the simulated imaging system and that anisoplanatic effects are present in the simulated images. These results are summarized in Table 3.1 and samples of the simulated images are shown in Figure 3.1.

Image formation in the spatial domain is the convolution of the atmospheric point-spread function (PSF) and the object intensity distribution.

$$
i\left(\vec{x}_{i}\right)=o\left(\vec{x}_{i}\right) * h\left(\vec{x}_{i}\right),
$$

where $\vec{x}_{i}$ is a two dimensional coordinate system in the image plane. Taking the Fourier transform of both sides of Equation 3.3 yields a multiplication in the Fourier spatial-frequency domain. The product of the complex object spectrum $O(\vec{f})$ with the atmospheric optical transfer function $(\mathrm{OTF}) H_{O T F}(\vec{f})$, results in the received complex 


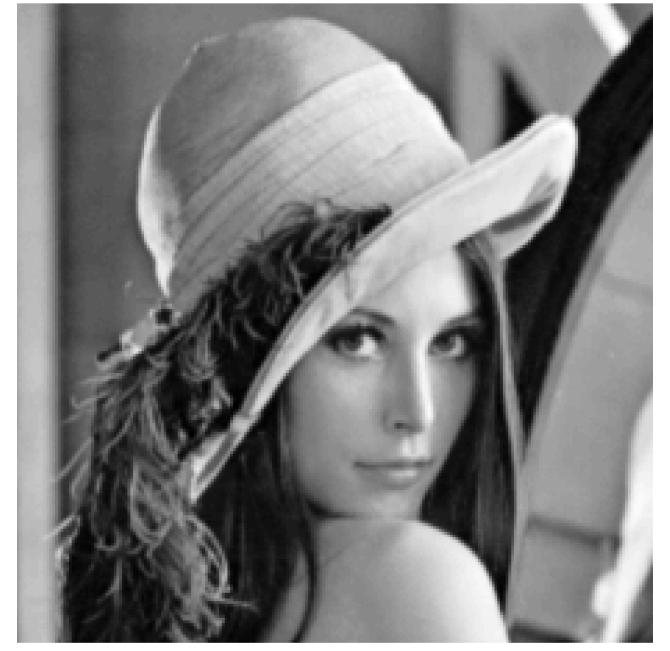

(a)

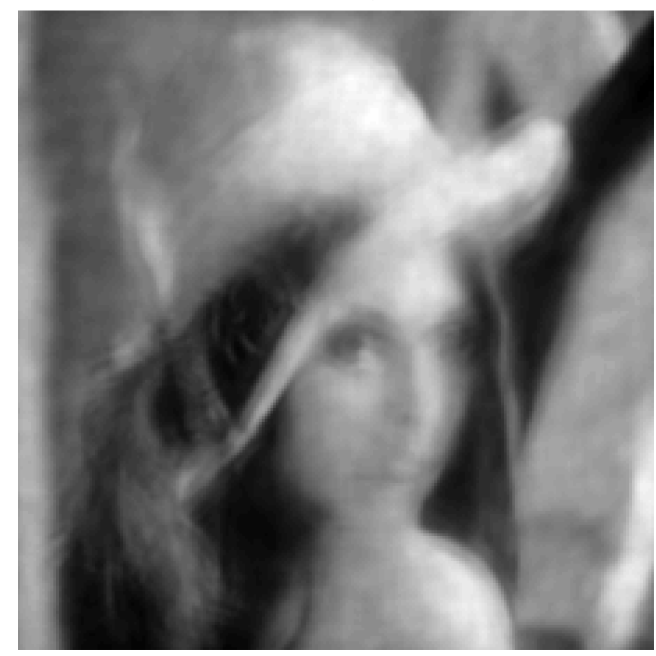

(c)

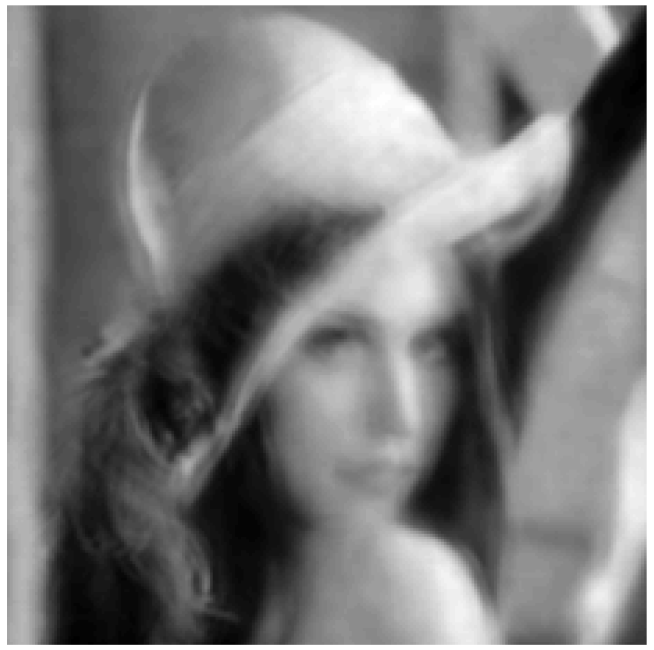

(b)

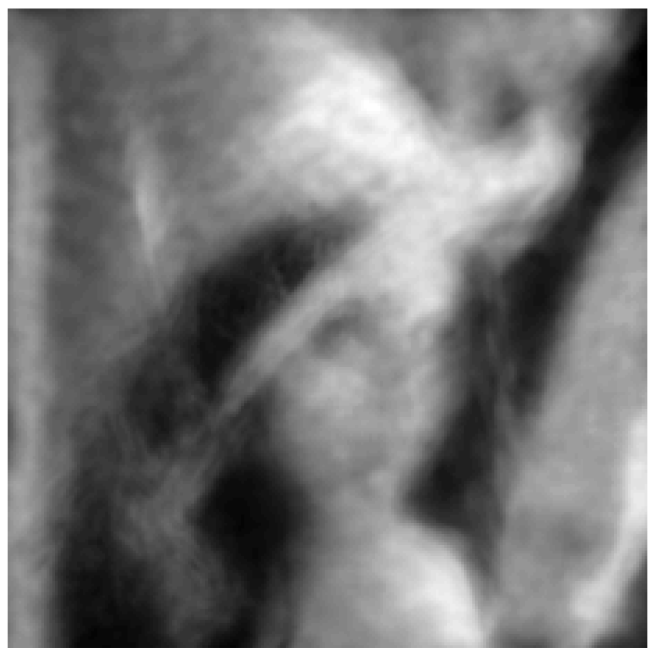

(d)

Figure 3.1: Horizontal Imaging Simulator Output. Single image representative samples of the horizontal image simulator output (a) Diffraction-Limited Image, (b) Image for $C_{N}^{2}=2.25 \times 10^{-14} m^{(-2 / 3)}$, (c) Image for $3.75 \times 10^{-14} m^{(-2 / 3)}$, (d) Image for $5.25 \times 10^{-14} m^{(-2 / 3)}$.

image spectrum $I(\vec{f})$.

$$
I(\vec{f})=O(\vec{f}) \times H_{O T F}(\vec{f})
$$

The modulus of the optical transfer function is very small, approaching zero, for spatial 
frequencies greater than $\frac{r_{0}}{\lambda \Delta z}$, causing the turbulent atmosphere to act like a low pass filter, limiting its ability to resolve small features.

\subsubsection{Speckle Imaging}

One strategy to mitigate the effects of atmospheric turbulence is the use of short exposure images to freeze the atmospheric turbulence. The term, "speckle imaging" arises from astronomical imaging, where short exposure images of isoplanatic objects exhibit high frequency intensity fluctuations, or "speckles". Two independent steps are required to recover the amplitude and phase information associated with the complex object spectrum. First, inverse Weiner filtering the ensemble average power spectral density yields the power spectrum of the object in the scene.

$$
\left\langle\left|I(\vec{f})^{2}\right|\right\rangle=\left\langle\left|H_{O T F}(\vec{f})\right|^{2}\right\rangle|O(\vec{f})|^{2}
$$

where $H_{O T F}(\vec{f})$ represents the long exposure optical transfer function of the imaging system and turbulent atmosphere, and $\langle\bullet\rangle$ represents the ensemble average of the argument. $\left\langle\left|H_{O T F}(\vec{f})\right|^{2}\right\rangle$ is positive and nonzero for all spatial frequencies up to the diffraction limit of the imaging system [5].

$$
|O(\vec{f})|^{2}=\frac{\left\langle\left|I(\vec{f})^{2}\right|\right\rangle}{\left\langle\left|H_{O T F}(\vec{f})\right|^{2}\right\rangle+\alpha}
$$


Astronomical observations determine $\left\langle\left|H_{O T F}(\vec{f})\right|^{2}\right\rangle$ through the use of a nearby guide star or artificial beacon as a point source. For my processing, the theoretical long-exposure atmospheric OTF of the simulated imaging system is applied to estimate the amplitude of the complex object spectrum[21], [23]. The additive term $\alpha$ is a constant adjusted by the user to account for low SNR at high spatial frequencies. The amplitude of the Fourier transform alone is insufficient to form an image. A separate process is required to recover the the phase information in the speckle images.

The object phase information is recovered by computing the bispectrum. The bi-spectrum is defined as

$$
B\left(\vec{f}_{1}, \Delta \vec{f}\right)=I\left(\vec{f}_{1}\right) I(\Delta \vec{f}) I^{*}\left(\vec{f}_{1}+\Delta \vec{f}\right)
$$

The object phase information is recovered from the bispectrum by substituting the image spectrum $I(\vec{f})$ defined in Equation 3.4 into the the definition in Equation 3.7 .

$$
\begin{aligned}
B\left(\vec{f}_{1}, \Delta \vec{f}\right)= & O\left(\vec{f}_{1}\right) O(\Delta \vec{f}) O\left(\vec{f}_{1}+\Delta \vec{f}\right) H_{O T F}\left(\vec{f}_{1}\right) \\
& \times H_{O T F}(\Delta \vec{f}) H_{O T F}\left(\vec{f}_{1}+\Delta \vec{f}\right)
\end{aligned}
$$


We can express the complex valued OTF in terms of magnitude and phase to yield

$$
\begin{aligned}
B\left(\vec{f}_{1}, \Delta \vec{f}\right)= & \left|O\left(\vec{f}_{1}\right) O(\Delta \vec{f}) O\left(\vec{f}_{1}+\Delta \vec{f}\right)\right| \times \\
& \left|H_{\text {OTF }}\left(\vec{f}_{1}\right) H_{O T F}(\Delta \vec{f}) H_{O T F}\left(\vec{f}_{1}+\Delta \vec{f}\right)\right| \\
& \times \exp \left\{j \left[\phi_{o}\left(\vec{f}_{1}\right)+\phi_{o}(\Delta \vec{f})-\phi_{o}\left(\vec{f}_{1}+\Delta \vec{f}\right)+\right.\right. \\
& \phi_{H}\left(\vec{f}_{1}+\phi_{H}(\Delta \vec{f})-\phi_{H}\left(\vec{f}_{1}+\Delta \vec{f}\right)\right.
\end{aligned}
$$

Because the amplitude is recovered in separate processing, we can reduce the number of significant terms in Equation 3.9 by setting all the amplitude terms to unity, leaving only the exponential phase terms in the reduced expression.

$$
\begin{aligned}
\left\langle\exp \left\{j\left[\phi_{B}\left(\vec{f}_{1}, \Delta \vec{f}\right)\right]\right\}\right\rangle_{N}= & \left\langle\operatorname { e x p } \left\{j \left[\phi_{o}\left(\vec{f}_{1}\right)+\phi_{o}(\Delta \vec{f})-\right.\right.\right. \\
& \left.\left.\phi_{o}\left(\vec{f}_{1}+\Delta \vec{f}\right)\right]\right\} \\
& \times \exp \left\{j \left[\phi_{H}\left(\vec{f}_{1}\right)+\phi_{H}(\Delta \vec{f})-\right.\right. \\
& \left.\left.\left.\phi_{H}\left(\vec{f}_{1}+\Delta \vec{f}\right)\right]\right\}\right\rangle_{N}
\end{aligned}
$$

Taking the expectation of Equation 3.10 over $\mathrm{N}$ frames it can be shown that the transfer function is real and that $\left\langle\phi_{H}\left(\vec{f}_{1}\right)+\phi_{H}\left(\vec{f}_{1}+\Delta \vec{f}\right)\right\rangle=0$ [18], to yield.

$$
\left\langle\exp \left\{j \hat{\phi}_{o}\left(\vec{f}_{1}+\Delta \vec{f}\right)\right\}\right\rangle=\left\langle\exp j\left\{\hat{\phi}_{o}\left(\vec{f}_{1}\right)+\hat{\phi}_{o}(\Delta \vec{f})-\hat{\phi}_{B}\left(\vec{f}_{1}, \Delta \vec{f}\right)\right\}\right\rangle
$$

This allows further simplification of Equation 3.7 to yield an estimate of the object phase at 
any arbitrary spatial frequency $\vec{f}^{\prime}=\vec{f}+\Delta \vec{f}$ in terms of the bispectrum estimate, assuming the phase at $\vec{f}$ and $\Delta f$ are known.

$$
\hat{\phi}_{o}\left(\vec{f}^{\prime}\right)=\hat{\phi}_{o}(\vec{f})+\hat{\phi}_{o}(\Delta \vec{f})-\hat{\phi}_{B}(\vec{f}, \Delta \vec{f}),
$$

Because images are real valued, the phase of $\vec{f}=0$ is zero. The adjacent frequency points $(0, \Delta f),(0,-\Delta f),(\Delta f, 0),(-\Delta f, 0)$ can also arbitrarily be set to zero without loss of image quality. This assumption will result in loss of image registration information and reconstructed images are often not centered within the image frame. Further processing can recenter the reconstructed images. Starting with these five known phase points it is possible to work outward from the origin and recover the phase for the entire image recursively.

\subsubsection{MFBD: Gaussian Noise Model}

MFBD is another common image reconstruction method [4] that uses an iterative process to recover object amplitude and phase information. A set of images that have been corrupted by additive Gaussian noise can be described.

$$
\left.d_{k}(x)=i\left(\vec{x}_{i}\right)\right)+n_{k}\left(\vec{x}_{i}\right)=o\left(\vec{x}_{i}\right) * h_{k}\left(\vec{x}_{i}, \vec{\alpha}_{k}\right)+n_{k}\left(\vec{x}_{i}\right)
$$


where $n_{k}\left(\vec{x}_{i}\right)$ represents an additive noise term characterized by an independent, identically distributed Gaussian random variable with zero mean and variance $\sigma^{2}$. The term $i\left(\vec{x}_{i}\right)$ is the noise free image characterized in Equation 3.3 as the convolution of a deterministic object and the imaging system's incoherent point spread function. The incoherent point spread function $h\left(\vec{x}_{i}\right)$ is the modulus squared of the coherent point spread function.

$$
h\left(\vec{x}_{i}\right)=\left|g\left(\vec{x}_{i}\right)\right|^{2}
$$

where the coherent point spread function is given by

$$
g\left(\vec{x}_{i}\right)=\sum_{\overrightarrow{x_{p}}} A e^{j \theta\left(\vec{x}_{p}\right)} e^{j \frac{2 \pi}{N}\left(\vec{x}_{i} \bullet \overrightarrow{x_{p}}\right)}
$$

where $A$ is a binary aperture function whose value is zero outside the aperture, $\overrightarrow{x_{p}}$ is a two dimensional coordinate system in the aperture plane, the $\bullet$ operator represents the inner product, and

$$
\theta\left(\overrightarrow{x_{p}}\right)=\sum \phi_{k}\left(\overrightarrow{x_{p}}\right) \alpha_{k}
$$

The phase term $\theta\left(\overrightarrow{x_{p}}\right)$ represents the accumulated phase perturbation in the aperture of the imaging system decomposed to a linear sum of orthonormal basis functions, $\phi_{k}\left(\overrightarrow{x_{p}}\right)$, and $\alpha_{k}$ are the weighting coefficients for each term and image. Zernike polynomials are a convenient set of basis functions commonly used in describing classical aberrations in 
optical systems. Using a Gaussian noise model, each point $x$ in each image $d_{k}\left(\vec{x}_{i}\right)$ is a random variable with a Gaussian Probability Density Function (PDF). The PDF of $d_{k}\left(\vec{x}_{i}\right)$ is parameterized by the object intensities $o\left(\vec{x}_{i}\right)$ and the vector of aberration weighting coefficients $\vec{\alpha}_{k}[11]$.

$$
p\left[d_{k}\left(\vec{x}_{i}\right) ; i\left(\vec{x}_{i}, \vec{\alpha}_{k}\right)\right]=\frac{1}{\left(2 \pi \sigma_{n}^{2}\right)^{1 / 2}} \exp \left\{-\frac{\left[d_{k}\left(\vec{x}_{i}\right)-i_{k}\left(\vec{x}_{i}, \vec{\alpha}_{k}\right)\right]^{2}}{2 \pi \sigma_{n}^{2}}\right\}
$$

and the likelihood of the complete data set consisting of all the pixel intensities in all the corrupted images is given by

$$
p\left[\left\{d_{k}\left(\vec{x}_{i}\right)\right\} ; i\left(\vec{x}_{i}, \vec{\alpha}_{k}\right)\right]=\prod_{k=1}^{K} \prod_{x \varepsilon \chi} \frac{1}{\left(2 \pi \sigma_{n}^{2}\right)^{1 / 2}} \exp \left\{-\frac{\left[d_{k}\left(\vec{x}_{i}\right)-i_{k}\left(\vec{x}_{i}, \vec{\alpha}_{k}\right)\right]^{2}}{2 \pi \sigma_{n}^{2}}\right\}
$$

Taking the natural log of Eqn. 3.18 makes the analysis more tractable. The resulting summation, neglecting a constant term, yields the log-likelihood function

$$
L\left(i\left(\vec{x}_{i}, \vec{\alpha}_{k}\right)\right)=-\sum_{k=1}^{K} \sum_{x \varepsilon \chi}\left[d_{k}\left(\vec{x}_{i}\right)-i\left(\vec{x}_{i}, \vec{\alpha}_{k}\right)\right]^{2}
$$

To maximize this cost function, the optimization routine needs at least an estimate of the gradient, $\nabla\left(i\left(\vec{x}_{i}, \vec{\alpha}_{k}\right)\right)$ and Hessian, $\nabla^{2}\left(i\left(\vec{x}_{i}, \vec{\alpha}_{k}\right)\right)$, of the likelihood function. Although an analytic form of the Hessian is not required, optimization is much more efficient if an analytic form of the gradient is provided. With respect to the pixel intensities the gradient 
of the Gaussian Log-Likelihood function[34] can be represented as:

$$
\left.\frac{\partial}{\partial o} L\left(i\left(\vec{x}_{i}, \vec{\alpha}\right)\right)=2 \sum_{k=1}^{K} \sum_{x \varepsilon \chi}\left[d_{k}\left(\vec{x}_{i}\right)\right)-\left(i_{k}\left(\vec{x}_{i}\right)\right)\right] \frac{\partial}{\partial o} i_{k}\left(\vec{x}_{i}\right)
$$

and the derivative of $i_{k}\left(\vec{x}_{i}\right)$ with respect to the object is given by

$$
\frac{\partial}{\partial o} i_{k}\left(\vec{x}_{i}\right)=\frac{\partial}{\partial o} \sum_{\vec{x}_{o}} h\left(\vec{x}_{i}-\vec{x}_{o}\right) o\left(\vec{x}_{i}\right)=h\left(\vec{x}_{i}-\vec{x}_{o}\right)
$$

where $h\left(x_{i}-x_{o}\right)$ is the incoherent point spread function. The gradient with respect to the Zernike coefficients is

$$
\begin{aligned}
& \left.\frac{\partial}{\partial \vec{\alpha}} L\left(i\left(\vec{x}_{i}, \vec{\alpha}\right)\right)=2 \sum_{k=1}^{K} \sum_{x \varepsilon \chi}\left[d_{k}\left(\vec{x}_{i}\right)\right)-\left(i_{k}\left(\vec{x}_{i}\right)\right)\right] \frac{\partial}{\partial \vec{\alpha}} i_{k}\left(\vec{x}_{i}, \vec{\alpha}_{k}\right) . \\
& \frac{\partial}{\partial \vec{\alpha}} i_{k}\left(\vec{x}_{i}, \vec{\alpha}_{k}\right)=\frac{\partial}{\partial \vec{\alpha}}\left(\sum_{\overrightarrow{x_{o}}} h\left(\vec{x}_{i}-\vec{x}_{o}\right) o\left(\vec{x}_{i}\right)\right)=\sum_{\overrightarrow{x_{o}}} \frac{\partial}{\partial \vec{\alpha}}\left(h\left(\vec{x}_{i}-\vec{x}_{o}\right) o\left(\vec{x}_{i}\right)\right)
\end{aligned}
$$

Since the object $o\left(\vec{x}_{i}\right)$ is constant with respect to the Zernike coefficients, Equation 3.23 reduces to

$$
\sum_{\overrightarrow{x_{o}}} \frac{\partial}{\partial \vec{\alpha}}\left(h\left(\vec{x}_{i}-\vec{x}_{o}\right) o\left(\vec{x}_{i}\right)\right)=\sum_{\overrightarrow{x_{o}}}\left(\frac{\partial}{\partial \vec{\alpha}} h\left(\vec{x}_{i}-\vec{x}_{o}\right)\right) o\left(\vec{x}_{i}\right)
$$

From equations 1.8, 1.9, and 1.10, we can express the derivative of the incoherent point 
spread function with respect to the $\alpha_{k}$ s in terms of the coherent point spread function,

$$
\frac{\partial}{\partial \vec{\alpha}}\left(h\left(\vec{x}_{i}-\vec{x}_{o}\right)\right)=\frac{\partial}{\partial \vec{\alpha}}\left|g\left(\vec{x}_{i}\right)\right|^{2}=\frac{\partial}{\partial \vec{\alpha}} g\left(\vec{x}_{i}\right) g^{*}\left(\vec{x}_{i}\right)=\left[\frac{\partial}{\partial \vec{\alpha}} g\left(\vec{x}_{i}\right)\right] g^{*}\left(\vec{x}_{i}\right)+g\left(\vec{x}_{i}\right)\left[\frac{\partial}{\partial \vec{\alpha}} g^{*}\left(\vec{x}_{i}\right)\right] .
$$

where ${ }^{*}(\bullet)$ represents the complex conjugate of the function in parenthesis. Equation 3.25 can be represented as

$$
2 \Re\left\{\left[\frac{\partial}{\partial \vec{\alpha}} g\left(\vec{x}_{i}\right)\right] g^{*}\left(\vec{x}_{i}\right)\right\},
$$

where $\Re(\bullet)$ takes the real part of a complex value.

$$
\begin{aligned}
& \frac{\partial}{\partial \vec{\alpha}} g\left(\vec{x}_{i}\right)=\frac{\partial}{\partial \vec{\alpha}}\left[\sum_{\overrightarrow{x_{p}}} A e^{j \theta\left(\overrightarrow{x_{p}}\right)} e^{j \frac{2 \pi}{N}\left(\vec{x}_{i} \bullet \overrightarrow{x_{p}}\right)}\right]=\sum_{\overrightarrow{x_{p}}}\left[j \frac{\partial \theta\left(\overrightarrow{x_{p}}\right)}{\partial \vec{\alpha}} A e^{j \theta\left(\overrightarrow{x_{p}}\right)} e^{j \frac{2 \pi}{N}\left(\vec{x}_{i} \bullet \overrightarrow{x_{p}}\right)}\right] \\
& \frac{\partial \theta\left(\overrightarrow{x_{p}}\right)}{\partial \vec{\alpha}}=\frac{\partial}{\partial \vec{\alpha}} \phi_{k}\left(\overrightarrow{x_{p}}\right) \alpha_{k}=\phi_{k}\left(\overrightarrow{x_{p}}\right)
\end{aligned}
$$

Combining the results of Equations 3.28, 3.27, 3.26 yields the final result

$$
\frac{\partial}{\partial \vec{\alpha}} i_{k}\left(\vec{x}_{i}, \vec{\alpha}_{k}\right)=\sum_{\vec{x}_{o}} 2 \Re\left\{\left[\sum_{\overrightarrow{x_{p}}}\left[j \phi_{k}\left(\vec{x}_{p}\right) A e^{j \theta\left(\vec{x}_{p}\right)} e^{j \frac{2 \pi}{N}\left(\vec{x}_{i} \bullet \overrightarrow{x_{p}}\right)}\right]\right] g^{*}\left(\vec{x}_{i}\right)\right\} o\left(\vec{x}_{i}\right)
$$




\subsubsection{MFBD: Poisson noise model}

Not all images are taken in full daylight. At low light levels, photon noise may dominate image frames. This is often characterized by a grainy quality of the images due to the random arrival times of discrete photons. Photon noise in images is described by modeling the number of photons detected in an image frame at each pixel as a Poisson random variable with a mean photon count rate $\lambda$, which is proportional to average pixel intensity. For this simulation the number of photons detected at each detector pixel is assumed to be an independent, Poisson distributed random variable with a mean rate given by a noiseless diffraction-limited image $i\left(\vec{x}_{i}\right)$. The random nature of the point spread function is neglected. The probability of detecting $d_{k}\left(\vec{x}_{i}\right)$ photons at a specific pixel location is given by

$$
p\left[d_{k}\left(\vec{x}_{i}\right)\right]=\frac{i\left(\vec{x}_{i}, \vec{\alpha}_{k}\right)^{d_{k}\left(\vec{x}_{i}\right)} e^{\left[-i_{k}\left(\vec{x}_{i}, \vec{\alpha}_{k}\right)\right]}}{d_{k}\left(\vec{x}_{i}\right) !}
$$

The distribution over the entire set of pixel locations $d_{k}$ is given by[11]

$$
p\left[\left\{d_{k}\right\}\right]=\prod_{k=1}^{K} \prod_{x \varepsilon \chi} \frac{i\left(\vec{x}_{i}, \vec{\alpha}_{k}\right) d_{k}\left(\vec{x}_{i}\right)}{e^{\left[-i_{k}\left(\vec{x}_{i}, \vec{\alpha}_{k}\right)\right]}}
$$

As before, taking the natural log yields a modified log-likelihood function

$$
L_{\text {poisson }}\left(i\left(\vec{x}_{i}, \vec{\alpha}\right)\right)=-\sum_{k=1}^{K} \sum_{x \varepsilon \chi}\left[d_{k}\left(\vec{x}_{i}\right) \ln \left(i_{k}\left(\vec{x}_{i}, \vec{\alpha}_{k}\right)\right)-i_{k}\left(\vec{x}_{i}\right)\right]-\sum_{k=1}^{K} \sum_{x \varepsilon \chi} d_{k}\left(\vec{x}_{i}\right)
$$


Where the last term is a constant and can be neglected. Taking the derivative with respect to the pixel intensities the gradient of the Poisson Log-Likelihood function[34] can be represented as:

$$
\frac{\partial}{\partial o} L_{\text {Poisson }}\left(i\left(\vec{x}_{i}, \vec{\alpha}\right)\right)=\sum_{k=1}^{K} \sum_{x \varepsilon \chi}\left[\frac{d_{k}\left(\vec{x}_{i}\right)}{i_{k}\left(\vec{x}_{i}, \vec{\alpha}_{k}\right)}-1\right] \frac{\partial}{\partial f} i_{k}\left(\vec{x}_{i}, \vec{\alpha}_{k}\right) .
$$

With respect to the Zernike coefficients the gradient of the Poisson Log-Likelihood function can be represented as:

$$
\frac{\partial}{\partial \vec{\alpha}} L_{\text {Poisson }}\left(i\left(\vec{x}_{i}, \vec{\alpha}\right)\right)=\sum_{k=1}^{K} \sum_{x \varepsilon \chi}\left[\frac{d_{k}\left(\vec{x}_{i}\right)}{i_{k}\left(\vec{x}_{i}, \vec{\alpha}_{k}\right)}-1\right] \frac{\partial}{\partial \vec{\alpha}} i_{k}\left(\vec{x}_{i}, \vec{\alpha}_{k}\right) .
$$

Where the $\frac{\partial}{\partial \vec{\alpha}}\left(i\left(\vec{x}_{i}, \vec{\alpha}\right)\right)$ was shown in Equation 3.29 .

\subsection{Methods}

My simulations assume that the propagation occurs over horizontally homogeneous conditions with both the object and the imaging system immersed in a turbulent atmosphere. Furthermore, I assume that the height above ground does not vary significantly between imaging system and the scene. Furthermore, $C_{n}^{2}$ is assumed to be constant over the propagation path [19]. I assume that the simulated data has effectively frozen the turbulence at the moment the turbulent image is created. Reconstruction processing begins 
by selecting images from the complete data set in groups of $N_{f}=2,4,6,8,10,12,14,16,18$, 20, 22, 23 and 25, 50, 75, 100, 125, 150, 175, and 200 frames. Once the number of frames to process is determined, that block size of images is incremented through the entire data set. The number of reconstructions used to determine the average MSE declines as the number of frames used in the reconstruction increases. The reduced number of trials for large frame groups is conjectured to be the reason why the MSE plots are not always monotonic. When 200 frames are applied to the reconstruction algorithm, only 5 trials are available.

Prior to applying the simulated turbulent images to the reconstruction algorithms, they must be recentered as tilt is not estimated in the MFBD algorithm. This was accomplished by using a correlation filter to compare each image in the stack to ensemble average image and then shifting the turbulent image to recenter it.

The recovered image was compared to the diffraction limited image and the mean square error is averaged over all pixels as follows

$$
M S E=\sum_{1}^{n} \frac{\left(i\left(\vec{x}_{i}\right)-\hat{i}\left(\vec{x}_{i}\right)\right)^{2}}{N^{2}}
$$

where $i\left(\vec{x}_{i}\right)$ is the normalized diffraction limited image, and $\hat{i}\left(\vec{x}_{i}\right)$ is the current normalized estimate of the object and $N^{2}$ is the total number of pixels in the image. The image stack was incremented to begin processing the next group of $K$ turbulent images and the process repeated. When the entire data set has been processed, the average of the vector of MSE's 
for images processed $K$ at a time is calculated. To facilitate direct comparison between the methods, exactly the same simulated turbulent images were applied to each estimator in every trial for both methods, and the MSE calculated from the recovered image.

In order to reduce edge effects associated with the finite support, each frame of the data set was preprocessed to pad the centered image by replicating the edges of the image outward and then adding a border of zeros. The abrupt transitions artificially introduced by the padding process can result in high spatial frequency artifacts in reconstructed images. The images are applied to the estimator were padded with 8 replicated pixels followed by 5 zero pixels at the margins of each image, bringing the total size of the image to $256 \times 256$ pixels.

The cost functions in Equations 3.19 and 3.32 are parameterized by the object pixel intensities and aberration coefficients, and are applied to a non-linear optimization technique to find the object and aberration coefficients most likely to have produced the images that were simulated in the data set. I am jointly processing all images and all Zernike coefficients, thus for a data set of $K, N \times N$ images, using $J$ Zernike polynomial terms, there will be $N^{2}+J \times K$ parameters that must be jointly estimated. To make the optimization tractable by reducing the run times, the limited-memory Broyden-Fletcher-Goldfarb-Shanno (L-BFGS) method is used to process the images. One of the drawbacks to searching along the gradient is the need for the Hessian $\nabla^{2} f(\vec{x})$. The limited memory form of the BFGS does not require an explicit expression for $\nabla^{2} f(\vec{x})$. It 
estimates the value of the Hessian matrix by maintaining the last few updates of $f(\vec{x})$ and $\nabla f(\vec{x})$. L-BFGS [32] is a quasi-Newtonian, "hill-climbing" technique that begins with an initial guess at a solution for $\vec{x}_{0}$ and then proceeds along a line in the direction $d_{k}$ pointed to by the gradient of the objective function evaluated at each location. Quasi-Newtonian line search optimization can quickly converge to a local minimum for cost functions, but there is no guarantee that that minimum is a global minimum. In processing the initial object estimate applied to the estimator was the average of all the frames used in the trial. Based on previous work [33], for the image reconstruction processing in this paper, the number of iterations was limited to 25 and the number of Zernike terms was fixed at 60 .

An initial guess at the object $o\left(\vec{x}_{0}\right)$ and phase parameters $\alpha_{i}$ is applied to the optimization routine. This initial guess is the spatial average of the $K$ frames being used in the estimate. The initial guess Zernike coefficients provided to the estimator are random Gaussian numbers with a mean of 0.5 and unity variance. 


\subsection{Comparison of Bispectrum and MFBD methods under Gaussian and Poisson noise models}

\subsubsection{Gaussian Noise Model}

\subsubsection{Case 1 Low Condition $C_{N}^{2}=2.25 \times 10^{-14} m^{(-2 / 3)}$}

Figure 3.2 shows the plots of mean square error for the Gaussian MFBD and bispectrum reconstructors, as the number of images applied to the estimator, $N_{f}$ are varied. For comparison, the average MSE for the entire data set is also provided. Examining Fig. 2.6 it can be seen that at $N_{f}=2$ and thereafter the estimator can be expected to perform better than the average mean square error for the simulated image. Marginal improvement in MSE declines at $N_{f}=12$, reaching a maximum improvement of approximately 40 percent over the average mean square error over the entire data set. However, if processing time is not of consequence, the MSE and its standard deviation continues to improve as additional images are added [33]. The diffraction limited image is compared to a sample of the simulated turbulent image data set images and a sample reconstructed object for MFBD and bispectrum are shown in Figure 3.3 . 


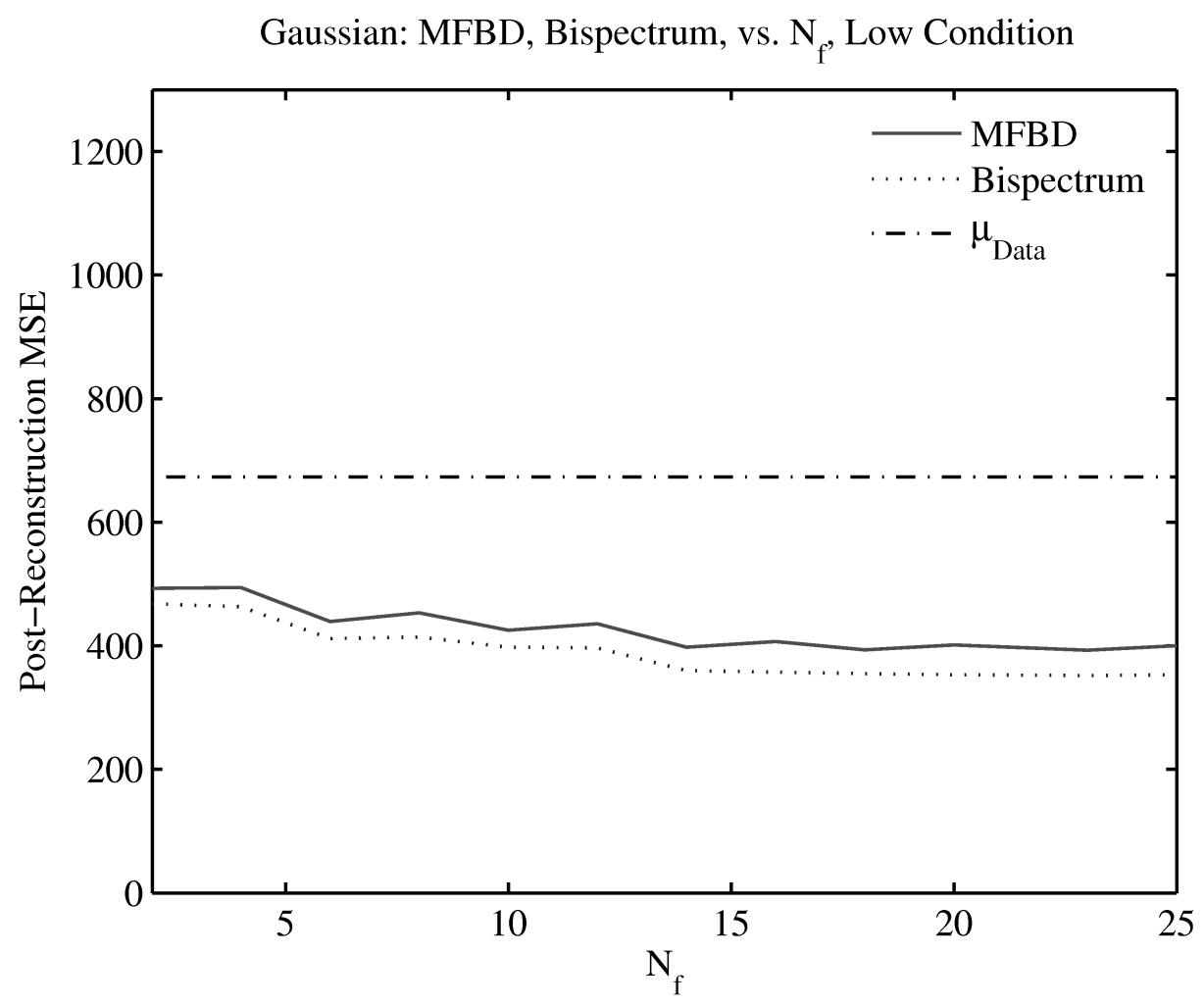

Figure 3.2: Comparison of Bispectrum and MFBD Mean Square Error as the number of input image frames is increased. For MFBD, $N_{z}=30$, and the number of iterations was fixed at 25. For Bispectrum, $N_{p}=5 \alpha=.3$. Case 1 Low turbulence $C_{N}^{2}=2.25 \times 10^{-14} m^{(-2 / 3)}$

\subsubsection{Case 2 Moderate Condition $C_{N}^{2}=3.75 \times 10^{-14} m^{(-2 / 3)}$}

Figure 3.4 shows (a) the diffraction limited image, (b) a sample recorded image, and (c) a sample reconstructed object. We see in Fig. 3.5 that the MFBD estimator will consistently perform on average better than the average image's error as soon as the size of the processing window reaches two frames. At $N_{f}=2$ and thereafter the estimator can be expected to perform better than the average mean square error of the simulated image. The improvement in MSE available by including additional input frames hits a maximum 


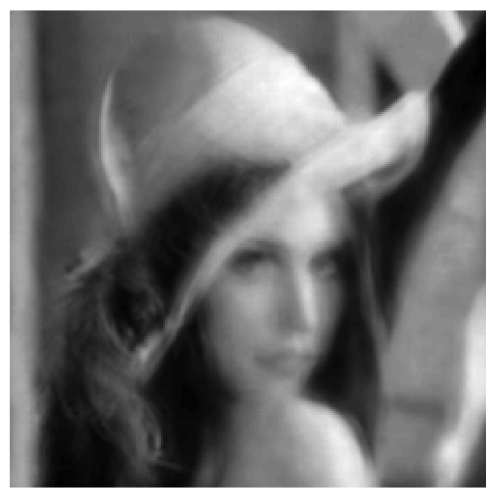

(a)

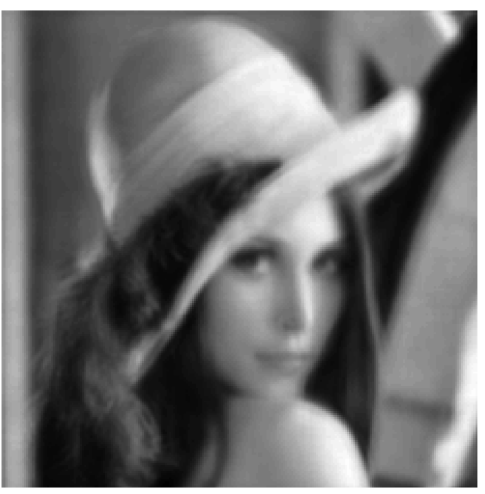

(b)

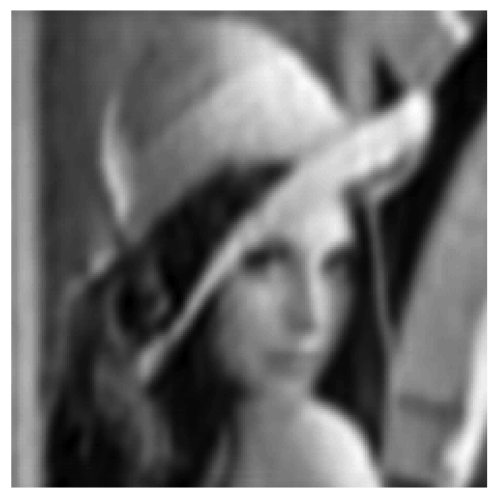

(c)

Figure 3.3: Case 1 sample images. Compares (a) a sample turbulent image (b) a sample MFBD reconstructed object and (c) a sample bispectrum reconstructed object.

of approximately 25 percent of full scale at $N_{f}=14$. Neither the MSE nor the standard deviation improves significantly as additional input images are added to the stack.

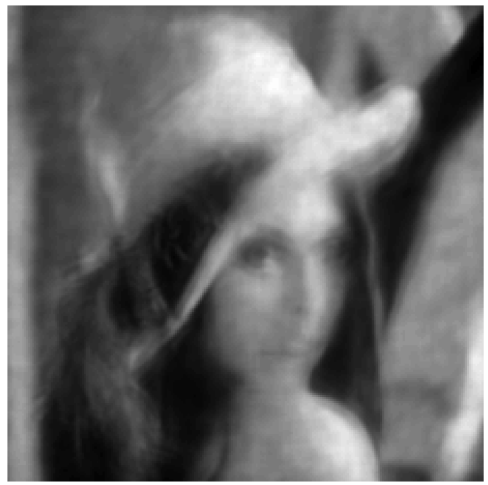

(a)

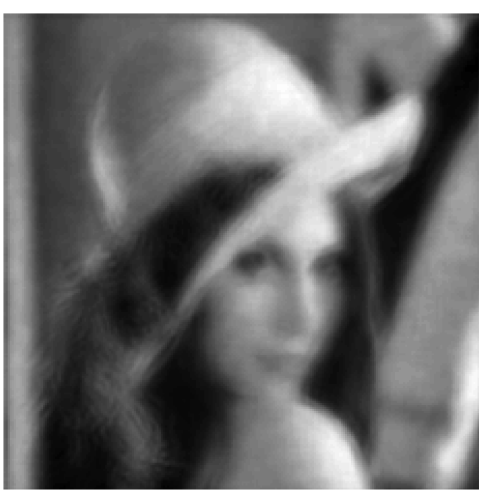

(b)

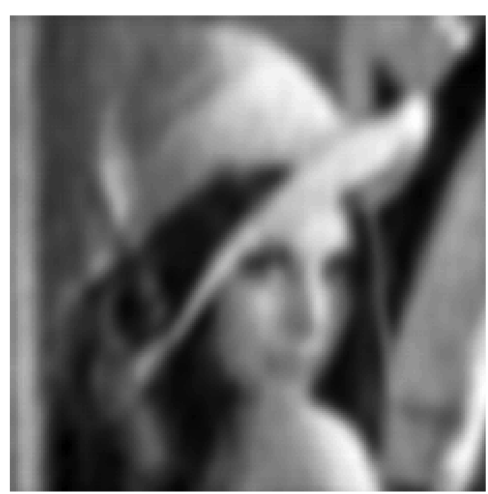

(c)

Figure 3.4: Case 2 sample images. Compares (a) a sample turbulent image (b) a sample MFBD reconstructed object and (c) a sample bispectrum reconstructed object. 


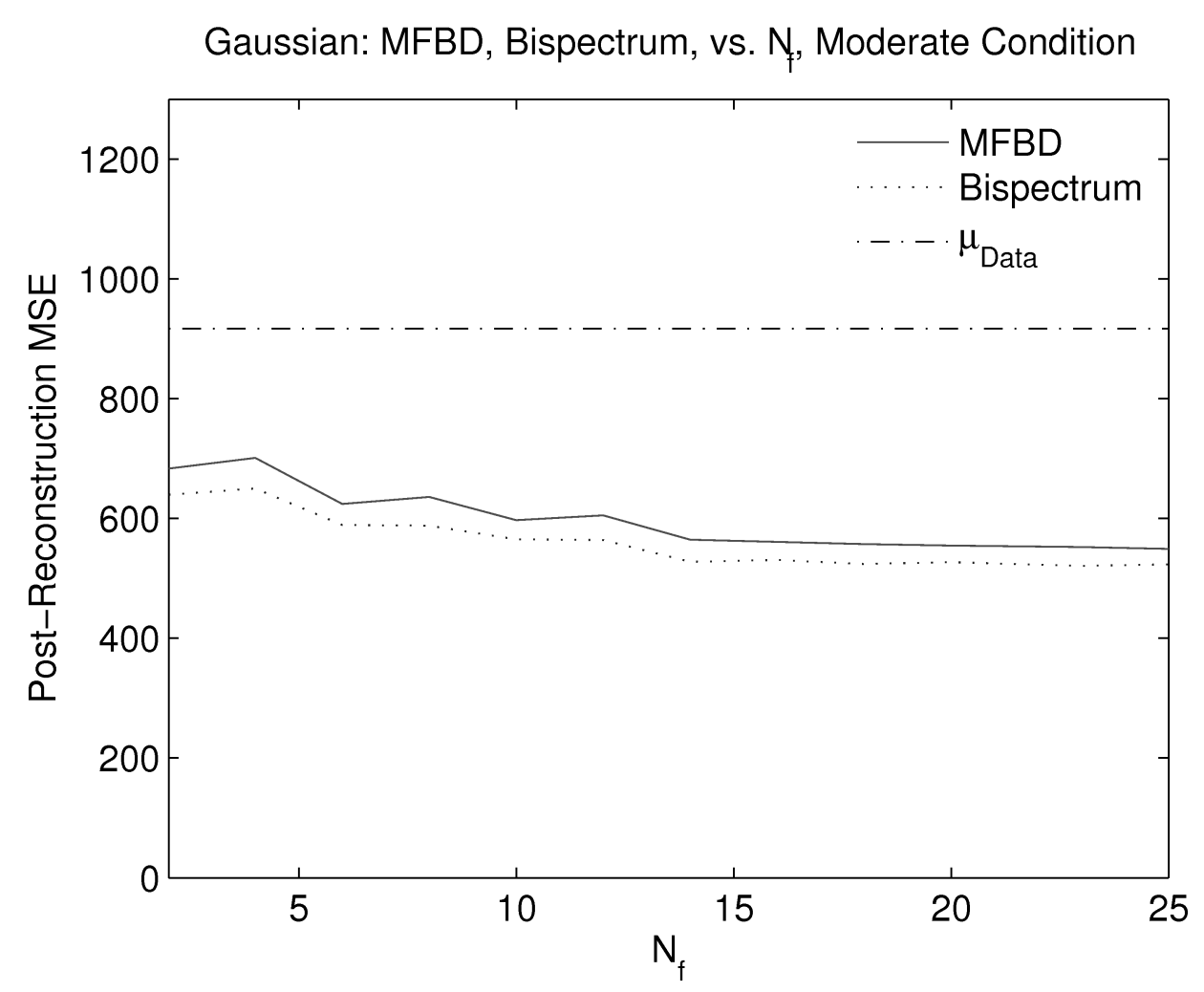

Figure 3.5: Comparison of Bispectrum and MFBD Mean Square Error as the number of input image frames is increased. For MFBD, $N_{z}=30$, and the number of iterations was fixed at 25. For Bispectrum, $N_{p}=5 \alpha=.3$. Case 2 Moderate turbulence $C_{N}^{2}=3.75 \times 10^{-14} m^{(-2 / 3)}$

\subsubsection{Case 3 Severe Condition $C_{N}^{2}=5.25 \times 10^{-14} m^{(-2 / 3)}$}

Again Figure 3.7 shows that the estimator requires at least two input frames to reliably produce an estimate of the object that has a lower mean square error than the average simulated image's MSE. At $N_{f}=2$ and thereafter the estimator can be expected to perform better than the average mean square error of the recorded image. The improvement in MSE available by including additional input frames hits a maximum of approximately 36 percent of full scale at $N_{f}=14$ and neither the MSE nor the standard deviation improves 
significantly as additional input images are added to the processing stack. Figure 3.6 shows (a) a sample recorded image, (b) a sample MFBD reconstructed object and (c) a sample bispectrum reconstructed object.

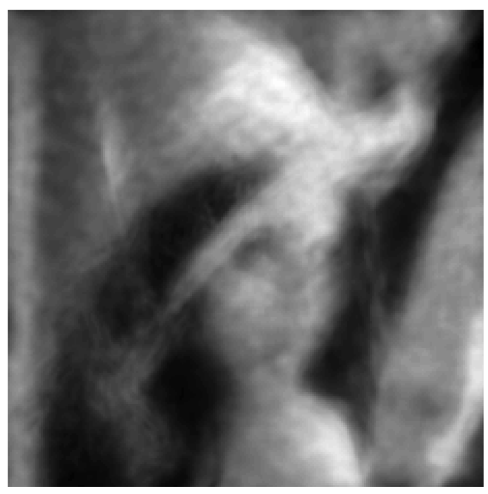

(a)

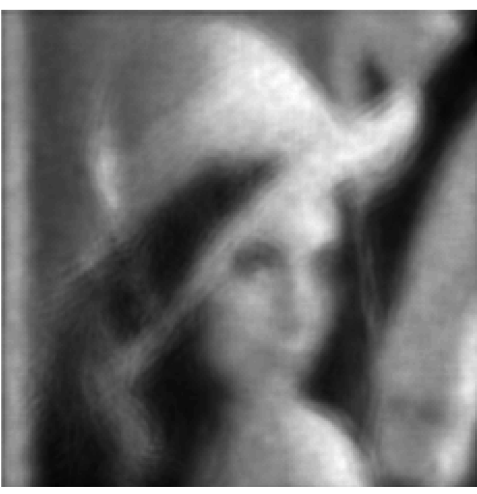

(b)

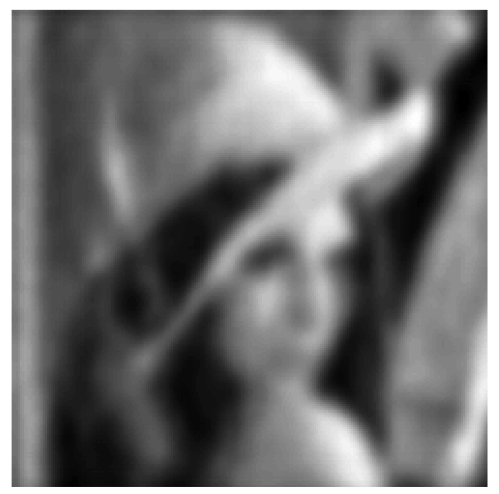

(c)

Figure 3.6: Case 3 sample images. Compares (a) a sample turbulent image (b) a sample MFBD reconstructed object and (c) a sample bispectrum reconstructed object.

\subsubsection{Poisson Noise Model Mean Photon Rate $2 \times 10^{6}, 30$ photons per pixel}

Each set of 1000 turbulent images, representing the the three turbulence cases was used to generate a set of speckle images with a mean photon count per image of $2 \times 10^{6}$. Each set of images was processed using the MFBD and bispectrum methods described above. The MFBD method used the cost function and gradients described in equations 3.16, 3.17, and 3.18 


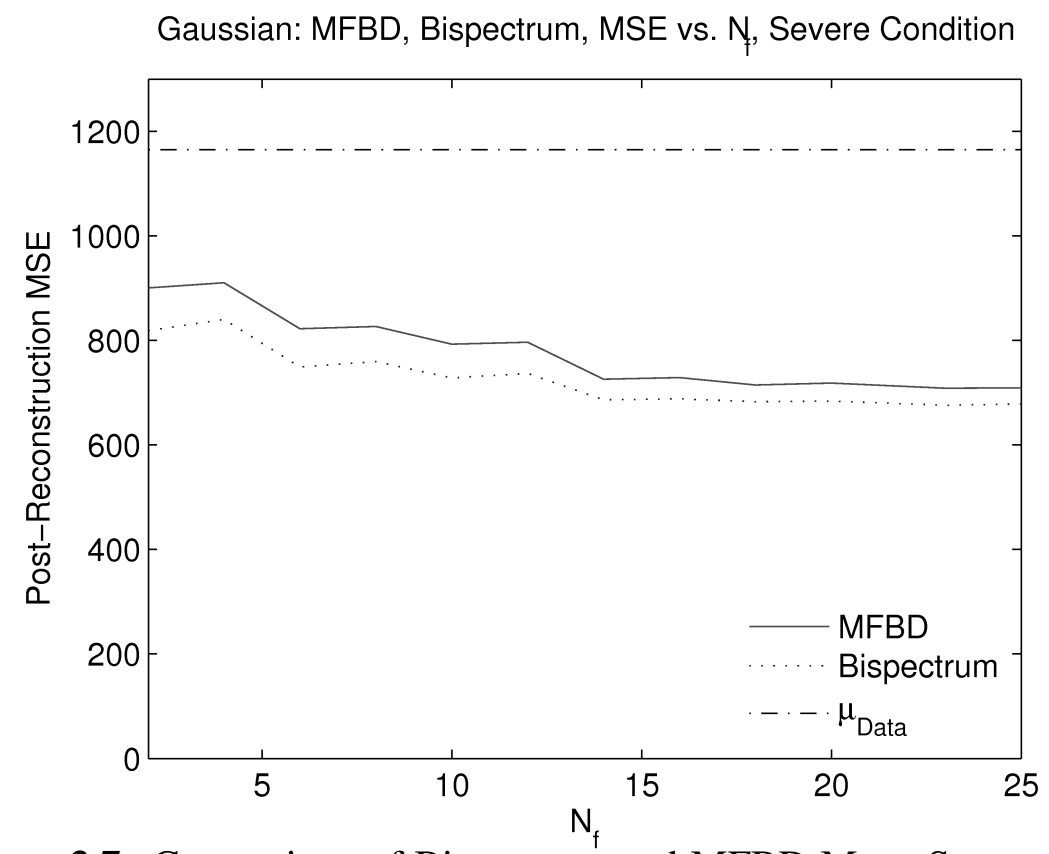

Figure 3.7: Comparison of Bispectrum and MFBD Mean Square Error as the number of input image frames is increased. For MFBD, $N_{z}=30$, and the number of iterations was fixed at 25. For Bispectrum, $N_{p}=5 \alpha=.3$. Case 3 Severe turbulence $C_{N}^{2}=5.25 \times 10^{-14} m^{(-2 / 3)}$

\subsubsection{Case 1 Low Condition $C_{N}^{2}=2.25 \times 10^{-14} m^{(-2 / 3)}$}

Figure 3.8 shows the plots of mean square error for the MFBD and bispectrum reconstructors, as the number of images, $N_{f}$ are varied. For comparison, the average MSE for the entire data set is also provided. On average, MFBD performance is less than the input images until 50 input frames are used in each reconstruction. At $N_{f}=50$ and thereafter the estimator can be expected to produce an estimate that has a value lower than 2095, the average mean square error of the images in the simulated data set. Marginal improvement in MSE continues as additional frames are added to the image stack reaching a maximum of about 38 percent improvement over the average MSE across the data set. 
Bispectrum achieves similar results with far fewer input frames. MFBD performance approximates that of speckle imaging techniques after 125 images are processed in each image stack. Processing times for the speckle imaging technique are much shorter than MFBD. A sample simulated turbulent image is compared to reconstructed objects in Fig. 3.9. The MFBD sample was processed using 175 frames, the bispectrum method used 200 frames in the reconstruction.

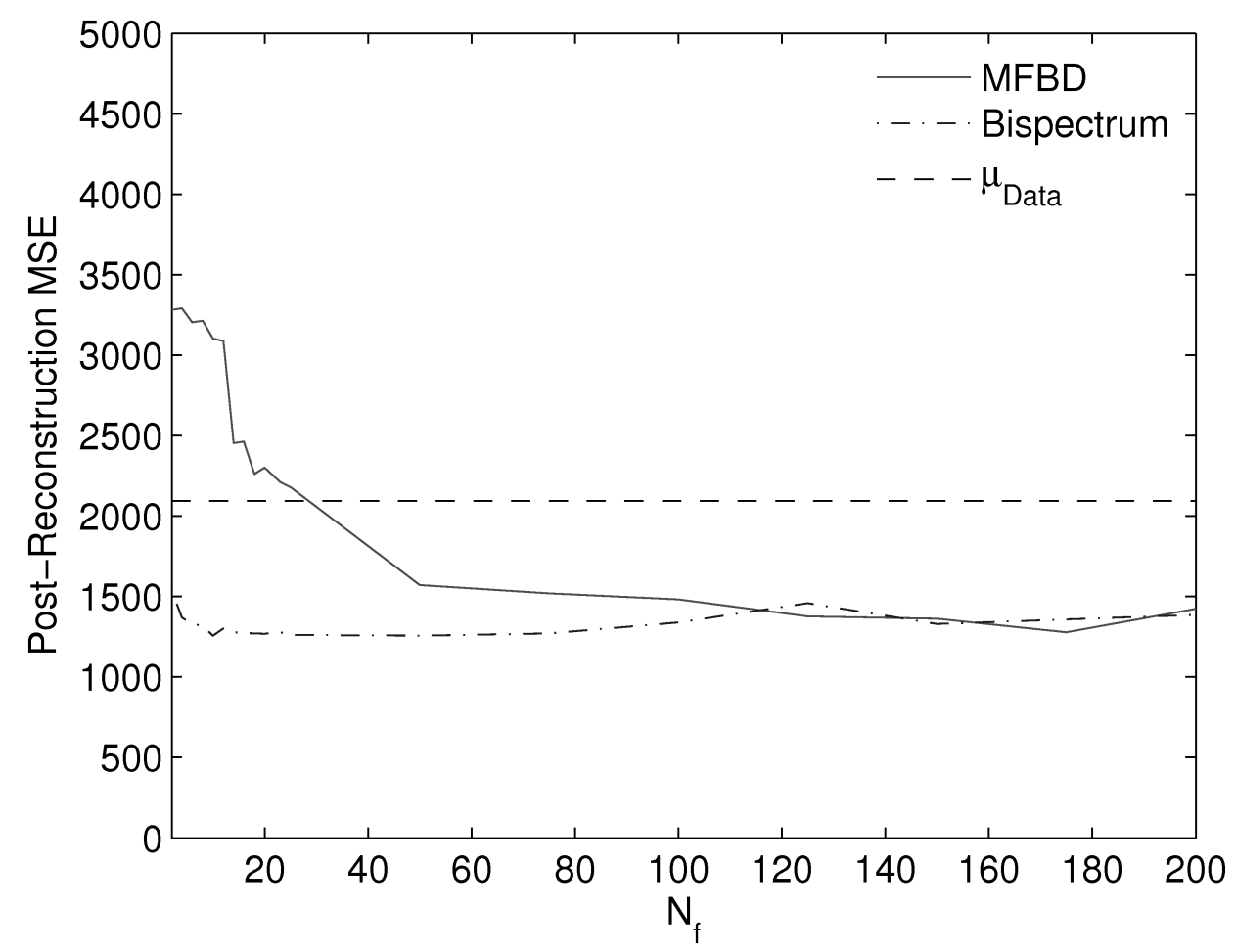

Figure 3.8: Comparison of Bispectrum and MFBD Mean Square Error as the number of input image frames is increased. For MFBD, $N_{z}=30$, and the number of iterations was fixed at 25. For Bispectrum, $N_{p}=5 \alpha=.3$. Poisson Case 1 Low condition $C_{N}^{2}=2.25 \times 10^{-14} m^{(-2 / 3)}$, mean photon rate $2 \times 10^{6}, 30$ photons per pixel 


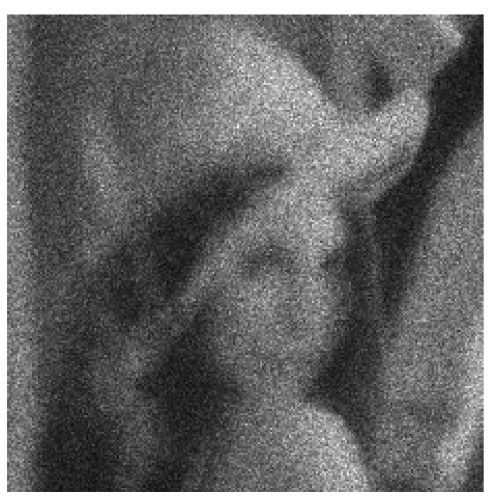

(a)

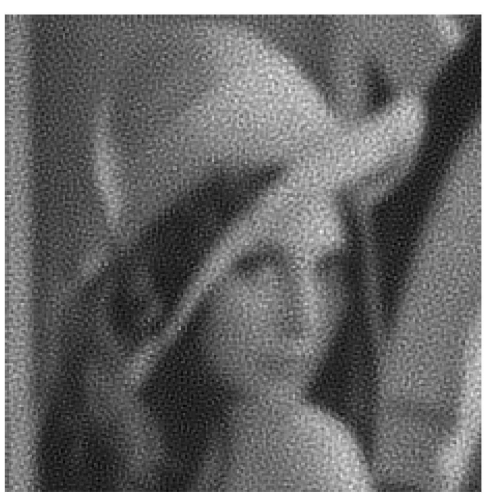

(b)

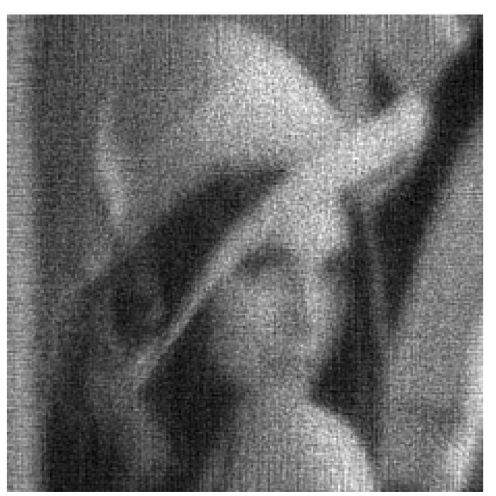

(c)

Figure 3.9: Case 1 Low condition sample images, Mean Photon Rate $2 \times 10^{6}, 30$ photons per pixel, $C_{N}^{2}=2.25 \times 10^{-14} m^{(-2 / 3)}$. Compares (a) a sample turbulent image (b) a sample MFBD reconstructed object $\left(N_{f}=\right.$ $175)$ and (c) a sample bispectrum $\left(N_{f}=200\right)$ reconstructed object. image.

\subsubsection{Case 2 Moderate Condition $C_{N}^{2}=3.75 \times 10^{-14} m^{(-2 / 3)}$}

Figure 3.10 shows (a) the diffraction limited image, (b) a sample recorded image, and (c) a sample reconstructed object using 175 frames to estimate the object. Again we see in Fig. 3.11 that the MFBD estimator will not perform on average any better than the average simulated turbulent image error until the number of images processed reaches 50 frames. At $N_{f}=50$ and thereafter the estimator can be expected to perform better than the average mean square error of the simulated image reaching a maximum of about 34 percent improvement. The marginal improvement in MSE available by including additional input frames begins to decline at about $N_{f}=175$ where MFBD processing begins to approximate speckle image processing performance. 


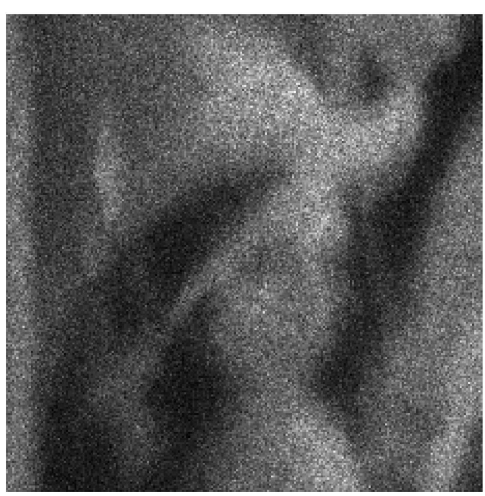

(a)

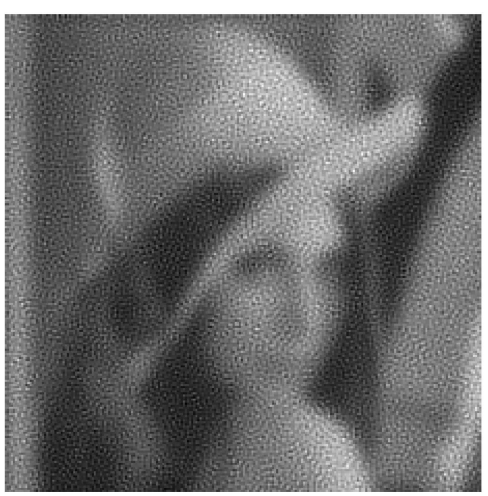

(b)

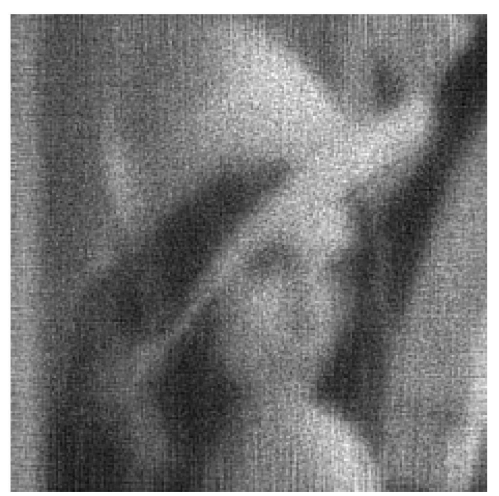

(c)

Figure 3.10: Case 2 Moderate condition sample images, Mean Photon Rate $2 \times 10^{6}, 30$ photons per pixel, $C_{N}^{2}=3.75 \times 10^{-14} m^{(-2 / 3)}$. Compares (a) a sample turbulent image (b) a sample MFBD reconstructed $\left(N_{f}=175\right)$ object and (c) a sample bispectrum $\left(N_{f}=200\right)$ reconstructed object.

\subsubsection{Case 3 Severe Condition $C_{N}^{2}=5.25 \times 10^{-14} m^{(-2 / 3)}$}

Again we see in Fig. 3.12 that the MFBD estimator will not perform on average any better than the average image error until the number of images offered to the estimator reaches 50 frames. At $N_{f}=50$ and thereafter the estimator can be expected to perform better than the average mean square error of the simulated image reaching a maximum of about 33 percent. MFBD performance may exceed that of speckle imaging after the number of frames processed exceeds 150. Figure 3.12 indicates that the MSE for speckle imaging increases. The number of frames is large with respect to the size of the data set, and only 5 trials were made with $N_{f}=200$. The marginal improvement in MSE available by including additional input frames begins to decline at about $\mathrm{N}=175$ and neither the MSE nor the standard deviation seems to improve significantly from there as additional input images are added to the stack. Figure 3.13 shows (a) a sample simulated turbulent image (b) a sample 


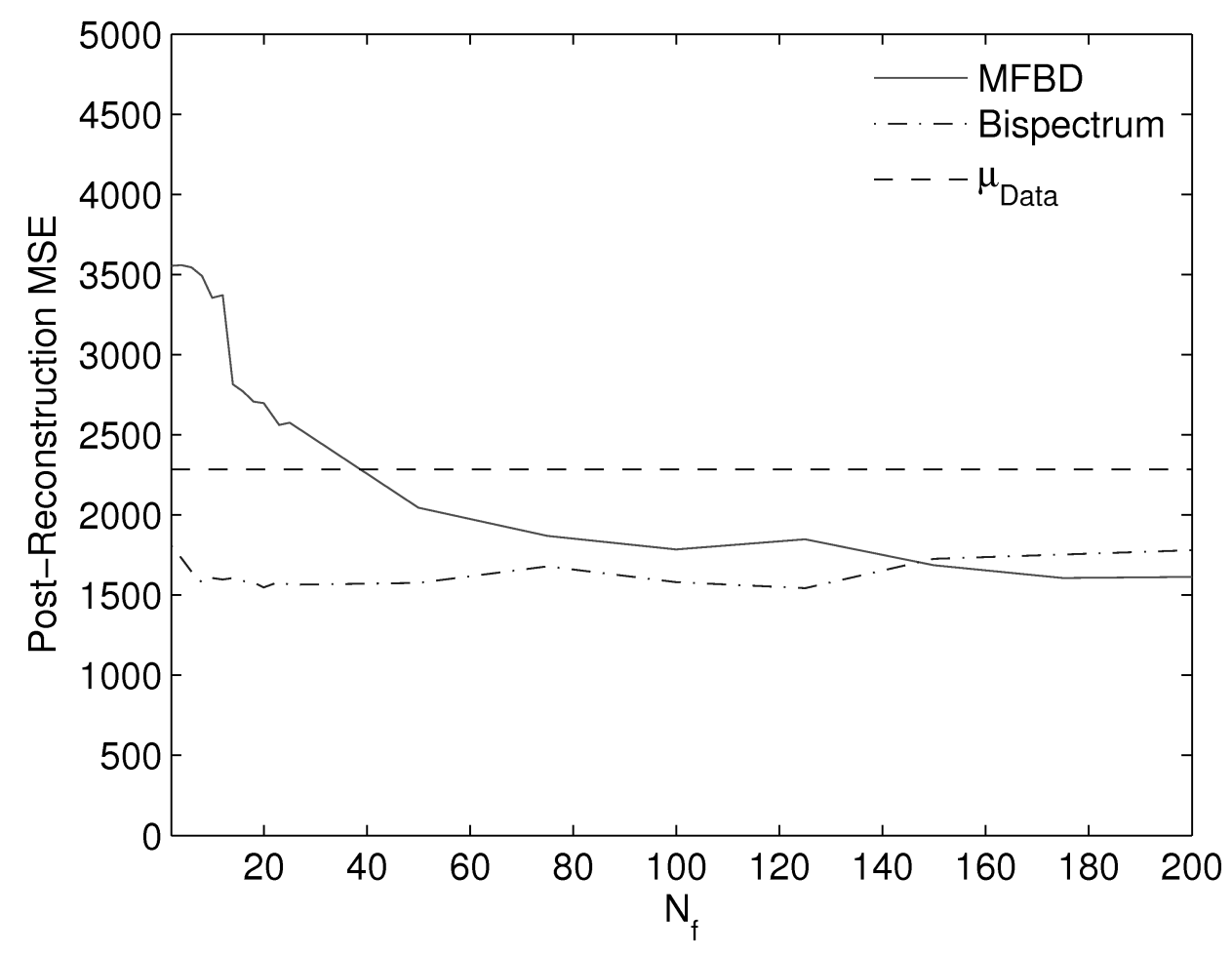

Figure 3.11: Comparison of Bispectrum and MFBD Mean Square Error as the number of input image frames is increased. For MFBD, $N_{z}=30$, and the number of iterations was fixed at 25. For Bispectrum, $N_{p}=5 \alpha=$ 3. Poisson Case 2 Moderate condition $C_{N}^{2}=3.75 \times 10^{-14} m^{(-2 / 3)}$, mean photon rate $2 \times 10^{6} 30$ photons per pixel

MFBD reconstricted object, $N_{f}=175$, and (c) a sample bispectrum reconstructed object $N_{f}=200$. 


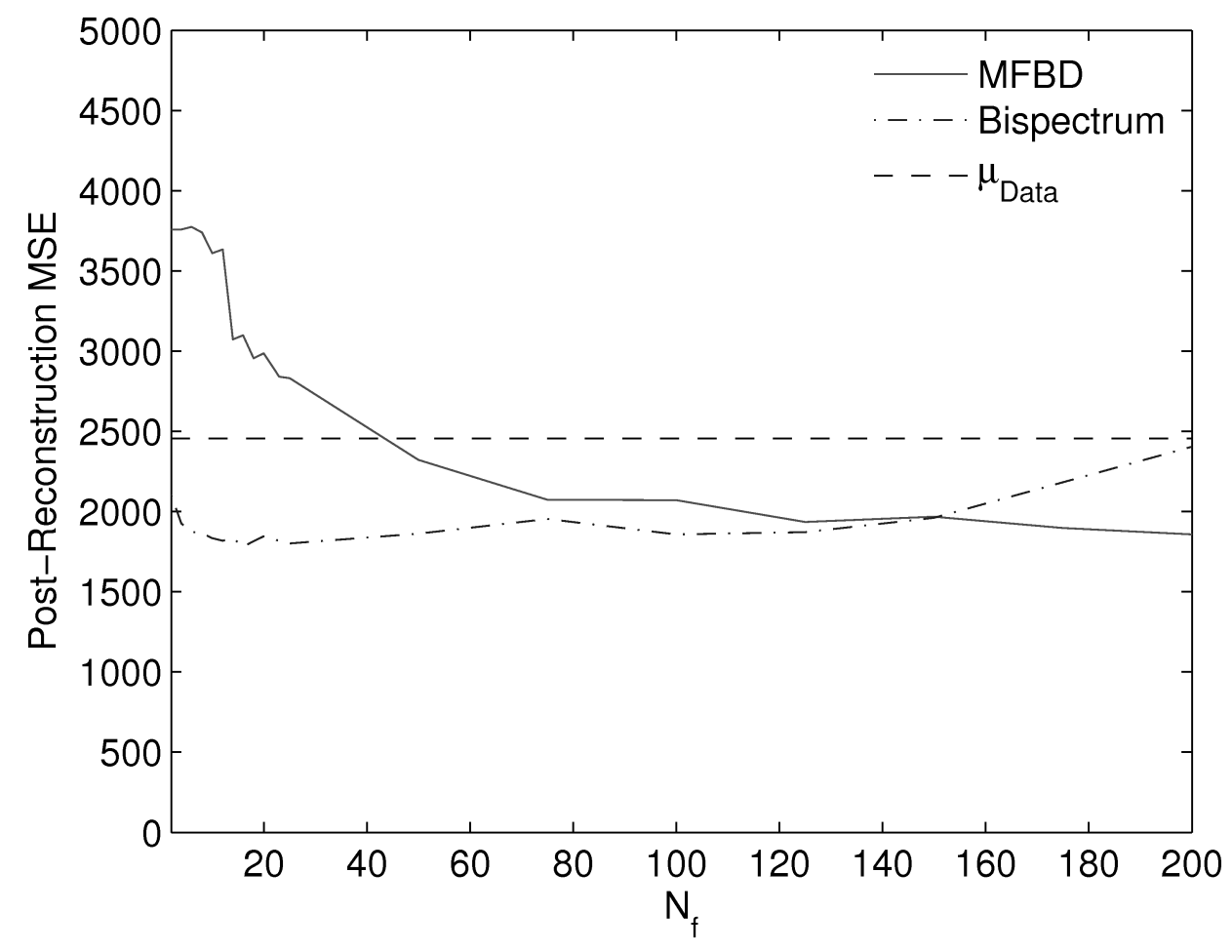

Figure 3.12: Comparison of Bispectrum and MFBD Mean Square Error as the number of input image frames is increased. For MFBD, $N_{z}=30$, and the number of iterations was fixed at 25. For Bispectrum, $N_{p}=5 \alpha=.3$. Poisson Case 3 Severe condition $C_{N}^{2}=2.25 \times 10^{-14} m^{(-2 / 3)}$, mean photon rate $2 \times 10^{6}, 30$ photons per pixel

\subsubsection{Poisson Noise Model Mean Photon Rate $1 \times 10^{6}, 15$ photons per pixel}

\subsubsection{Case 1 Low Condition $C_{N}^{2}=2.25 \times 10^{-14} m^{(-2 / 3)}$}

Figure 3.15 shows the plots of mean square error for the Poisson noise model MFBD, and bispectrum reconstructors, as the number of images, $N_{f}$ are varied. For comparison, the 


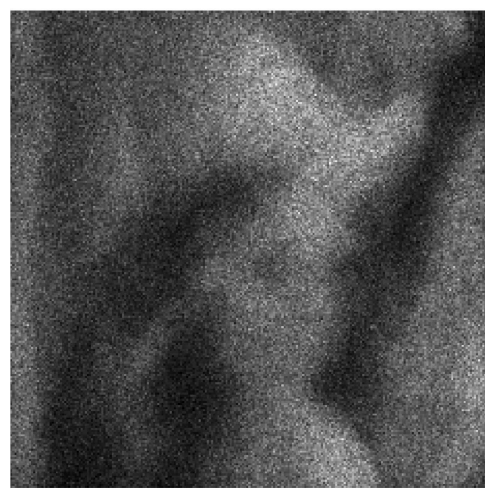

(a)

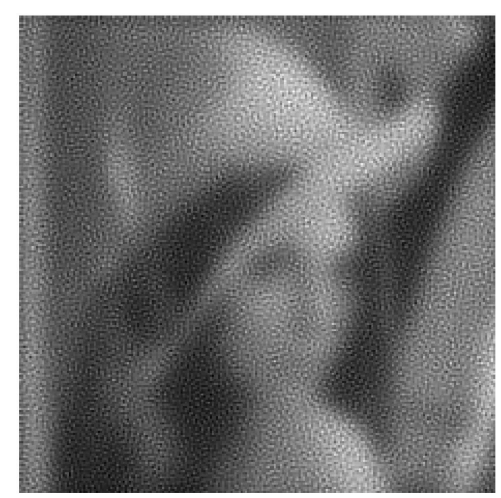

(b)

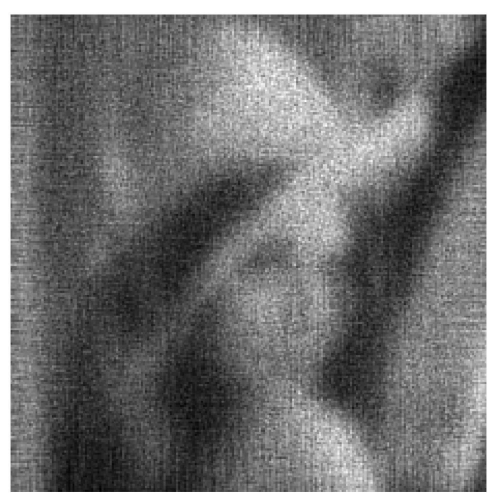

(c)

Figure 3.13: Case 3 Severe condition sample images, Mean Photon Rate $2 \times 10^{6}, C_{N}^{2}=5.25 \times 10^{-14} m^{(-2 / 3)}$. Compares (a) a sample simulated turbulent image (b) a sample MFBD reconstructed object, $N_{f}=175$, and (c) a sample bispectrum reconstructed object $N_{f}=200$

average MSE for the entire data set is also provided. On average, MFBD performance is less than the input images until 50 input frames are used in each reconstruction. At $N_{f}=50$ and thereafter the estimator can be expected to produce an estimate that has a value lower than the average mean square error of the images in the simulated data set. The performance of the MFBD estimator will on average exceed that of speckle imaging techniques at when the number of frames processed $N_{f}>50$. MFBD error reduces as additional frames are added to the image stack reaching a maximum of about 38 percent improvement over the average MSE across the data set. A sample simulated turbulent image is compared to reconstructed objects in Fig. 3.14. The MFBD sample was processed using 175 frames, the bispectrum method used 200 frames in the reconstruction. Processing times for bispectrum are significantly shorter than for MFBD. 


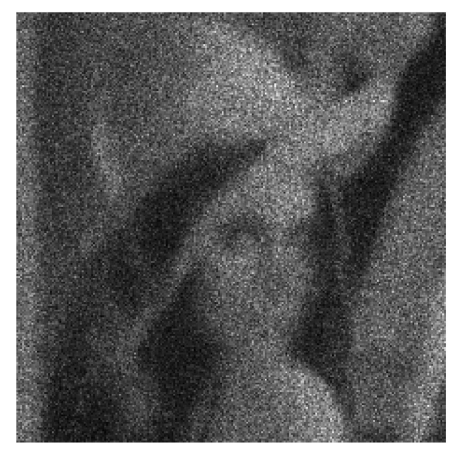

(a)

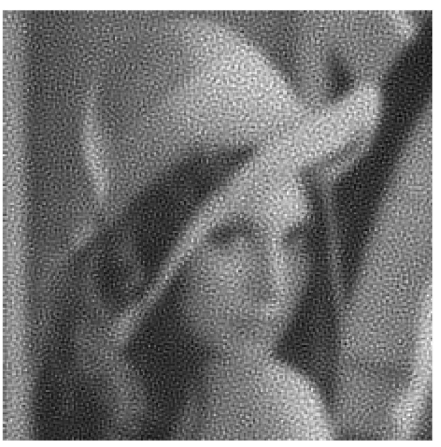

(b)

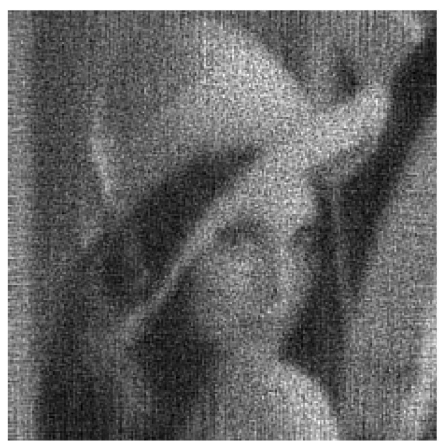

(c)

Figure 3.14: Case 1 Low condition $C_{N}^{2}=2.25 \times 10^{-14} m^{(-2 / 3)}$, mean photon rate $1 \times 10^{6}$. Compares (a) a sample simulated turbulent image (b) a sample MFBD reconstricted object, $N_{f}=175$, and (c) a sample bispectrum reconstructed object $N_{f}=200$

\subsubsection{Case 2 Moderate Condition $C_{N}^{2}=3.75 \times 10^{-14} m^{(-2 / 3)}$}

Figure 3.16 shows the plots of mean square error for the Poisson noise model MFBD and bispectrum reconstructors, as the number of images, $N_{f}$ are increased. For comparison, the average MSE for the entire data set is also provided. On average, MFBD performance is less than the input images until 50 input frames are used in each reconstruction. At $N_{f}=50$ and thereafter the estimator can be expected to produce an estimate that has a value lower than the average mean square error of the images in the simulated data set. The performance of the MFBD estimator will on average exceed that of speckle imaging techniques at when the number of frames processed $N_{f}>50$. MFBD error reduces as additional frames are added to the image stack reaching a maximum of about 38 percent improvement over the average MSE across the data set. A sample simulated turbulent image is compared to reconstructed objects in Fig. 3.17. The MFBD sample was processed using 175 frames, the 


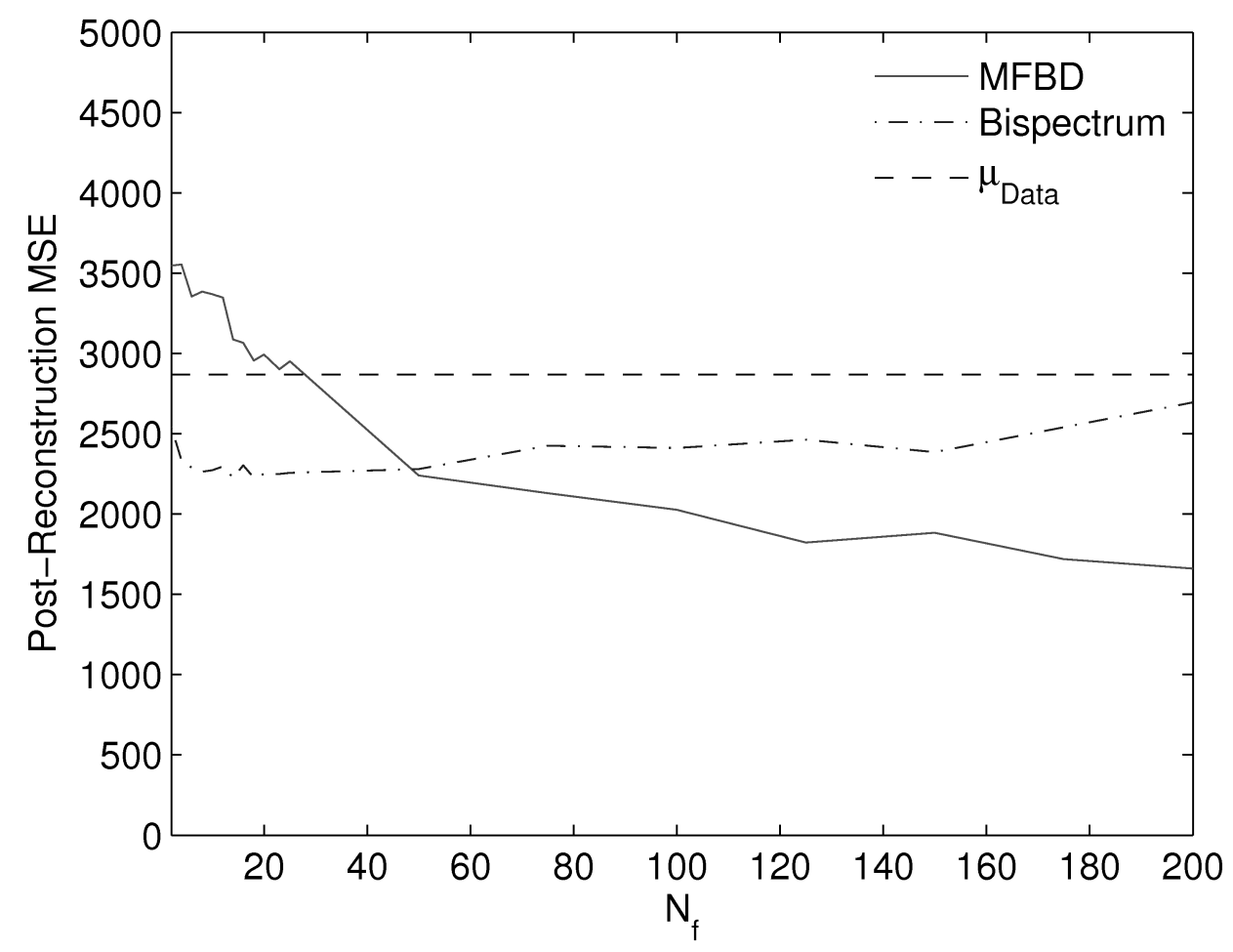

Figure 3.15: Comparison of Bispectrum and MFBD Mean Square Error as the number of input image frames is increased. For MFBD, $N_{z}=30$, and the number of iterations was fixed at 25. For Bispectrum, $N_{p}=5 \alpha=$ .3. Poisson Case 1 Low turbulence $C_{N}^{2}=2.25 \times 10^{-14}$, mean photon rate $1 \times 10^{6} 15$ photons per pixel

bispectrum method used 200 frames in the reconstruction. Processing times for bispectrum are significantly shorter than for MFBD.

\subsubsection{Case 3 Severe Condition $C_{N}^{2}=5.25 \times 10^{-14} m^{(-2 / 3)}$}

Figure 3.18 shows the plots of mean square error for the Poisson noise model MFBD and bispectrum reconstructors, as the number of images, $N_{f}$ are varied. For comparison, the average MSE for the entire data set is also provided. On average, MFBD performance is 


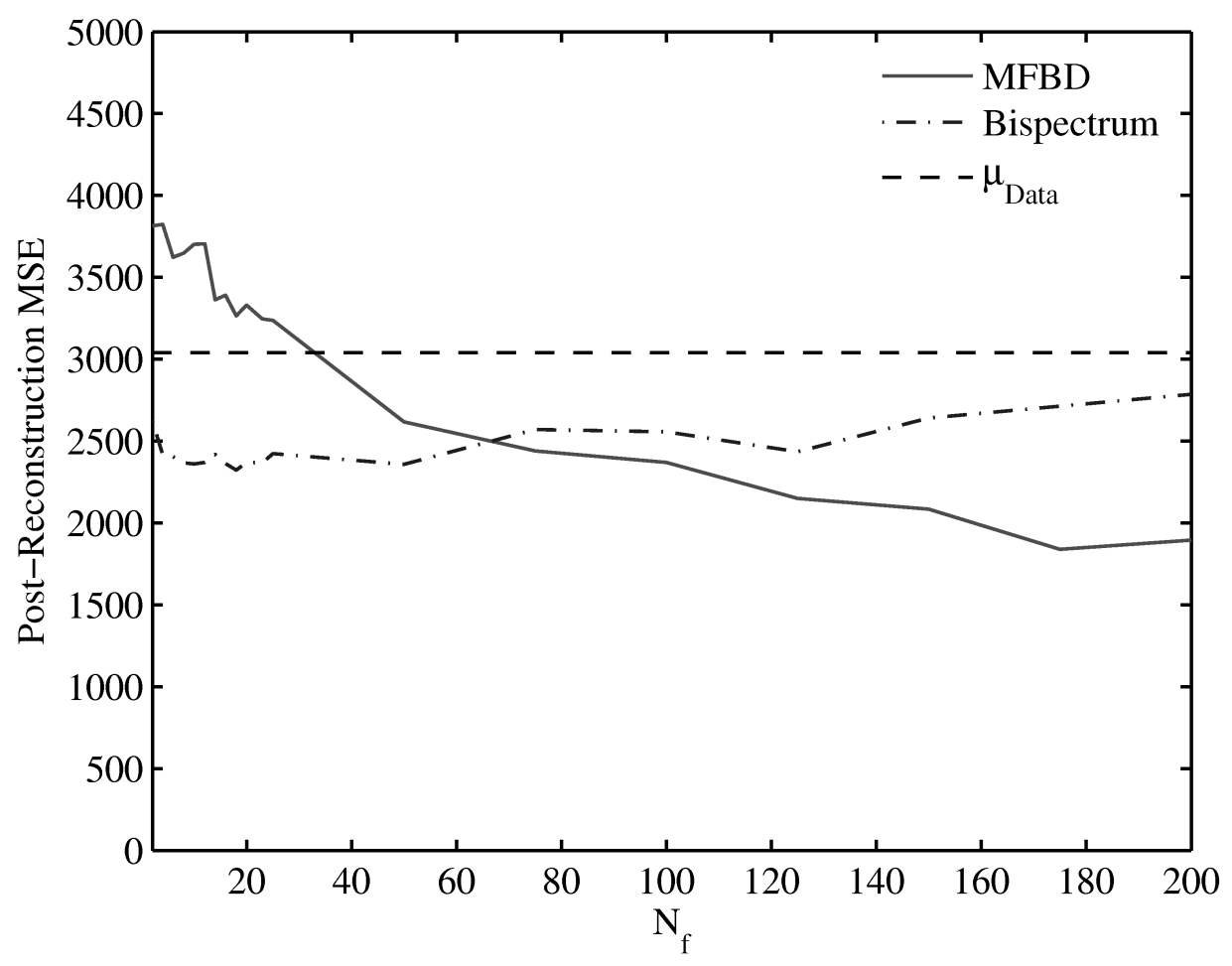

Figure 3.16: Comparison of Bispectrum and MFBD residual Mean Square Error as the number of input image frames is increased. For MFBD, $N_{z}=$ 30, and the number of iterations was fixed at 25. For Bispectrum, $N_{p}=5 \alpha$ $=.3$. Poisson Case 2 Moderate turbulence $C_{N}^{2}=3.75 \times 10^{-14} m^{(-2 / 3)}$, mean photon rate $1 \times 10^{6} 15$ photons per pixel

less than the input images until 50 input frames are used in each reconstruction. At $N_{f}=50$ and thereafter the estimator can be expected to produce an estimate that has a value lower than the average mean square error of the images in the simulated data set. The performance of the MFBD estimator will on average exceed that of speckle imaging techniques at when the number of frames processed $N_{f}>50$ until 125 frames are used in the reconstruction. for $N_{f}=125$ and 150, MFBD and Speckle techniques achieve approximately the same results. MFBD error reduces as additional frames are added to the image stack reaching a 


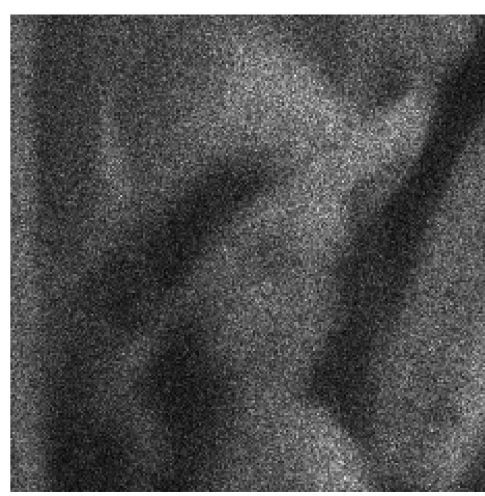

(a)

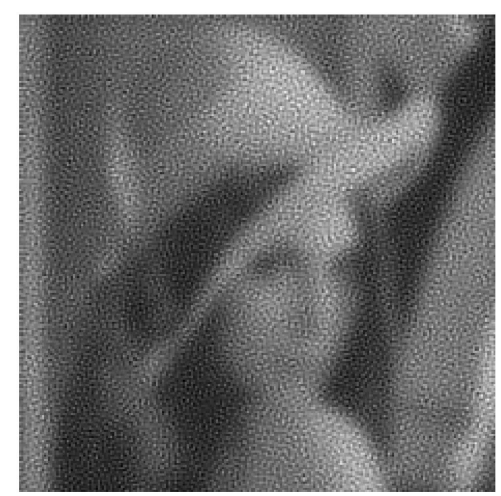

(b)

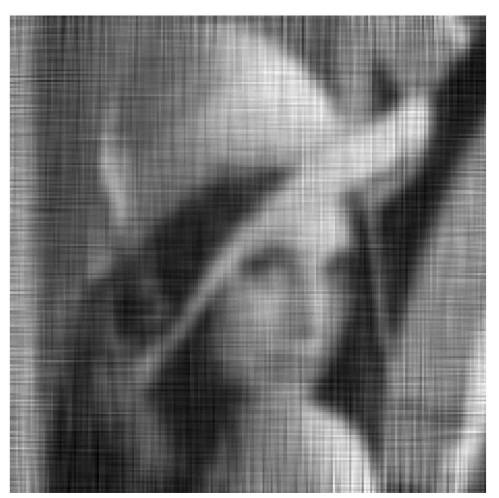

(c)

Figure 3.17: Case 2 Moderate turbulence $C_{N}^{2}=3.75 \times 10^{-14} m^{(-2 / 3)}$, mean photon rate $1 \times 10^{6}$.Compares (a) a sample turbulent image (b) a sample MFBD reconstructed object and (c) a sample bispectrum reconstructed object.

maximum of about 40 percent improvement over the average MSE across the data set. A sample simulated turbulent image is compared to reconstructed objects in Fig. 3.19. The MFBD sample was processed using 175 frames, the bispectrum method used 200 frames in the reconstruction.

\subsubsection{Poisson Noise Model Mean Photon Rate $5 \times 10^{5}, 8$ photons per pixel}

\subsubsection{Case 1 Low Condition $C_{N}^{2}=2.25 \times 10^{-14} m^{(-2 / 3)}$}

Figure 3.21 shows the plots of mean square error for the Poisson noise model MFBD and bispectrum reconstructors, as the number of images, $N_{f}$ is increased. For comparison, the 


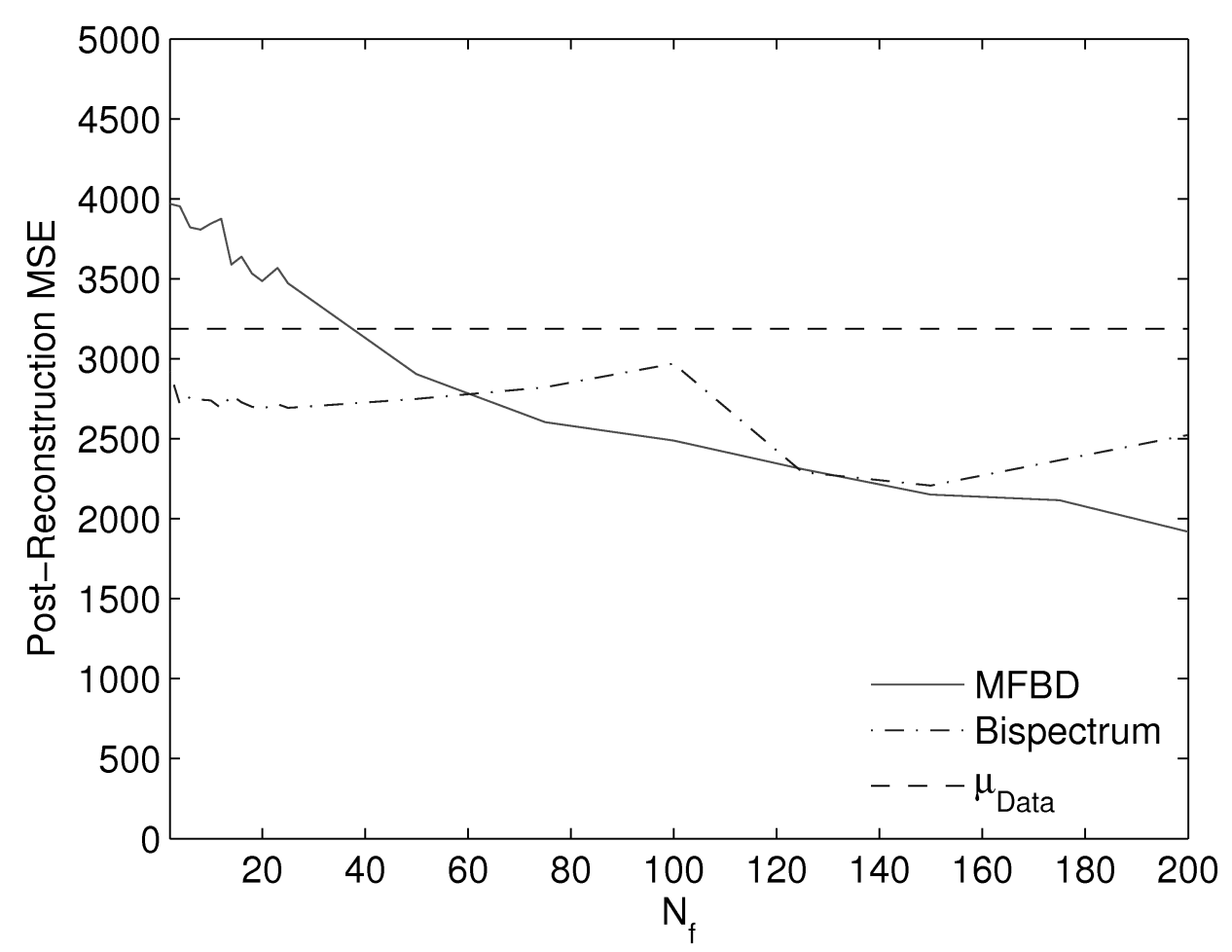

Figure 3.18: Bispectrum MFBD Mean Square Error vs. number of frames. Poisson Case 3 Severe turbulence $C_{N}^{2}=2.25 \times 10^{-14} m^{(-2 / 3)}$, mean photon rate $1 \times 10^{6} 15$ photons per pixel

average MSE for the entire data set is also provided. On average, MFBD performance is less than the input images until 25 input frames are used in each reconstruction. At $N_{f}=25$ and thereafter the estimator can be expected to produce an estimate that has a value lower than the average mean square error of the images in the simulated data set. The mean square error produced by the MFBD estimator will on average be less than that of speckle imaging techniques when the number of frames processed $N_{f}>100$. One possible reason that the speckle imaging estimator performance has diminished for large numbers of input frames is that the estimator was optimized for higher light levels. Only 5 bispectrum subplanes were used to reconstruct the phase estimate, which is sufficient for high light levels, but the 


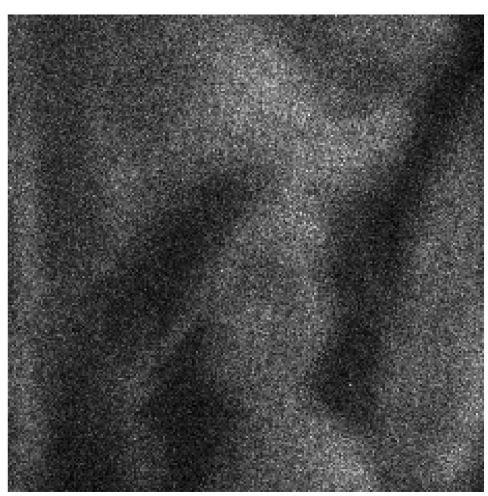

(a)

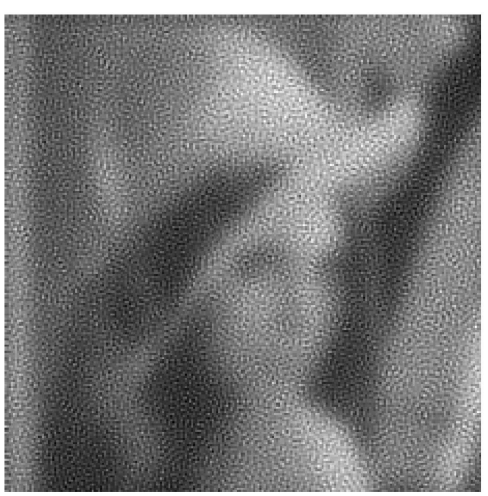

(b)

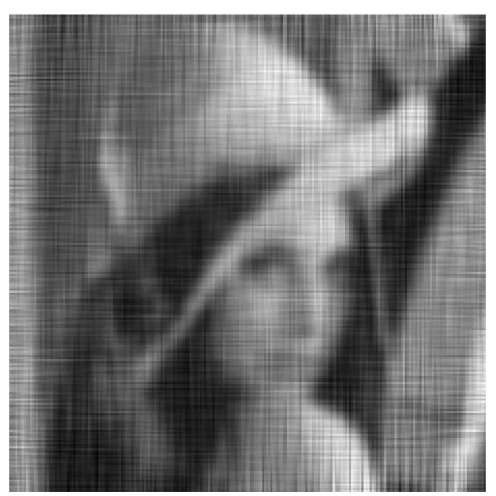

(c)

Figure 3.19: Case 3 Severe turbulence $C_{N}^{2}=5.25 \times 10^{-14} m^{(-2 / 3)}$, mean photon rate $1 \times 10^{6}$. Compares (a) a sample turbulent image (b) a sample MFBD reconstructed object and (c) a sample bispectrum reconstructed object.

inclusion of higher frequency offsets may increase the noise in the final estimate thereby degrading the MSE performance. After $N_{f}>125$ mean square error is not further reduced by MFBD processing. A sample simulated turbulent image is compared to reconstructed objects in Fig. 3.20. The MFBD sample was processed using 175 frames, the bispectrum method used 200 frames in the reconstruction.

\subsubsection{Case 2 Moderate Condition $C_{N}^{2}=3.75 \times 10^{-14} m^{(-2 / 3)}$}

Figure 3.23 shows the plots of mean square error for the Poisson noise model MFBD and bispectrum reconstructors, as the number of images, $N_{f}$ is increased. For comparison, the average MSE for the entire data set is also provided. On average, MFBD performance is less than the input images until 50 input frames are used in each reconstruction. The 


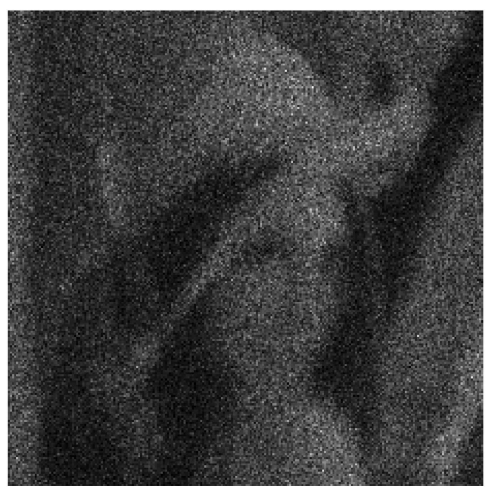

(a)

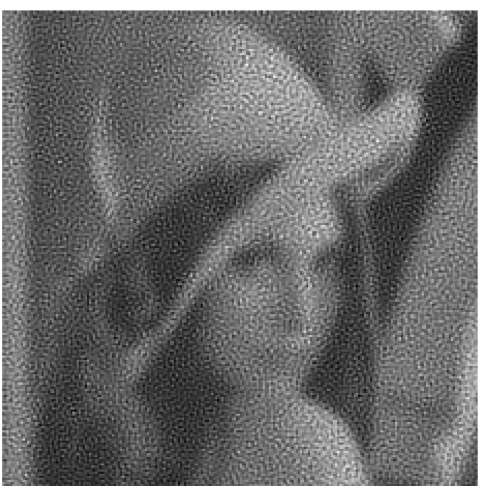

(b)

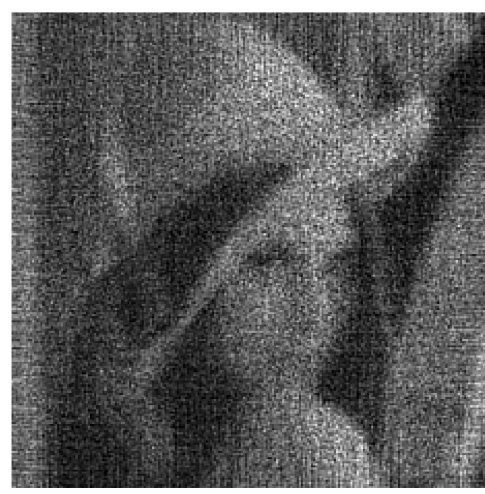

(c)

Figure 3.20: Case 1 Low turbulencs $C_{N}^{2}=2.25 \times 10^{-14} m^{(-2 / 3)}$, mean photon rate $5 \times 10^{5}$. Compares (a) a sample turbulent image (b) a sample MFBD reconstructed object $N_{f}=175$, and (c) a sample $N_{f}=200$, reconstructed object.

performance of the MFBD estimator will on average exceed that of speckle imaging techniques at when the number of frames processed $N_{f}>75$. MFBD error reduces as additional frames are added to the image stack. One possible reason that the speckle imaging estimator performance has diminished for large numbers of input frames is that the estimator was optimized for higher light levels. Only 5 bispectrum subplanes were used to reconstruct the phase estimate, which is sufficient for high light levels, but the inclusion of higher frequency offsets may increase the noise in the final estimate thereby degrading the MSE performance. A sample simulated turbulent image is compared to reconstructed objects in Fig. 3.22. The MFBD sample was processed using 175 frames, the bispectrum method used 200 frames in the reconstruction. Processing times for bispectrum are significantly shorter than for MFBD. 


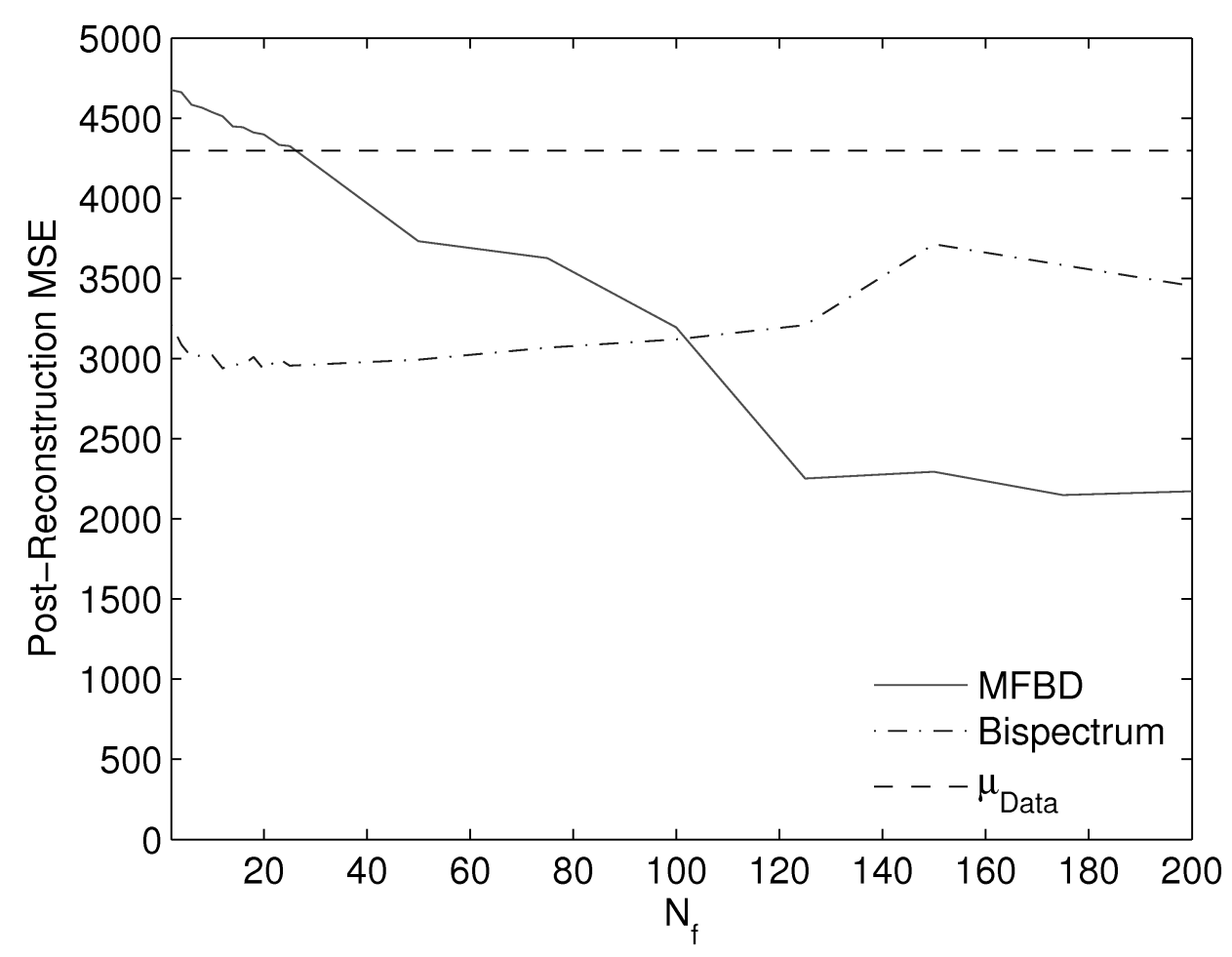

Figure 3.21: Comparison of Bispectrum and MFBD Mean Square Error as the number of input image frames is increased. For MFBD, $N_{z}=30$, and the number of iterations was fixed at 25. For Bispectrum, $N_{p}=5 \alpha=.3$. Poisson Case 1 Low turbulence $C_{N}^{2}=2.25 \times 10^{-14} m^{(-2 / 3)}$, mean photon rate $5 \times 10^{5}, 8$ photons per pixel

\subsubsection{Case 3 Severe Condition $C_{N}^{2}=5.25 \times 10^{-14} m^{(-2 / 3)}$}

Figure 3.25 shows the plots of mean square error for the Poisson noise model MFBD and bispectrum reconstructors, as the number of images, $N_{f}$ is increased. For comparison, the average MSE for the entire data set is also provided. The mean square error produced by the MFBD estimator will on average be less than that of speckle imaging techniques when the number of frames processed $N_{f}>50$. Mean square error is further reduced by 


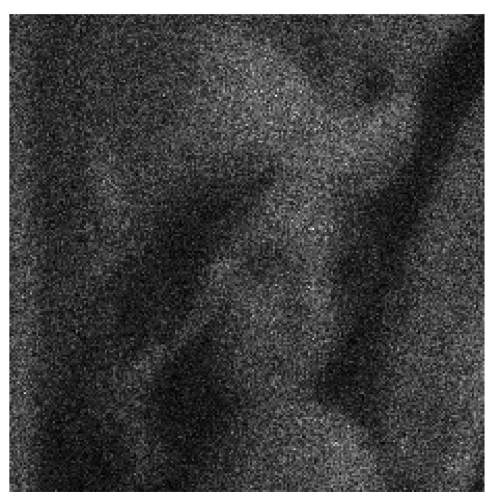

(a)

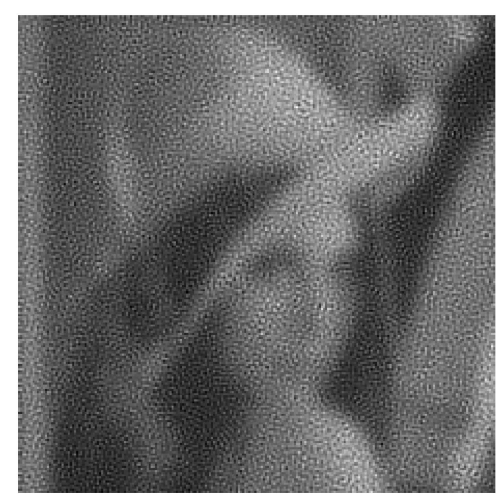

(b)

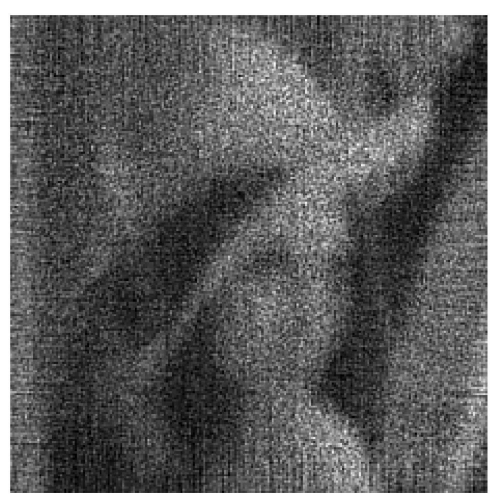

(c)

Figure 3.22: Case 2 Moderate turbulence $C_{N}^{2}=3.75 \times 10^{-14} m^{(-2 / 3)}$, mean photon rate $5 \times 10^{5}$. Compares (a) a sample turbulent image (b) a sample MFBD reconstructed object and (c) a sample bispectrum reconstructed object.

MFBD processing. One possible reason that the speckle imaging estimator performance has diminished for large numbers of input frames is that the estimator was optimized for higher light levels. Only 5 bispectrum subplanes were used to reconstruct the phase estimate, which is sufficient for high light levels, but the inclusion of higher frequency offsets may increase the noise in the final estimate thereby degrading the MSE performance. A sample simulated turbulent image is compared to reconstructed objects in Fig. 3.24. The MFBD sample was processed using 175 frames, the bispectrum method used 200 frames in the reconstruction. 


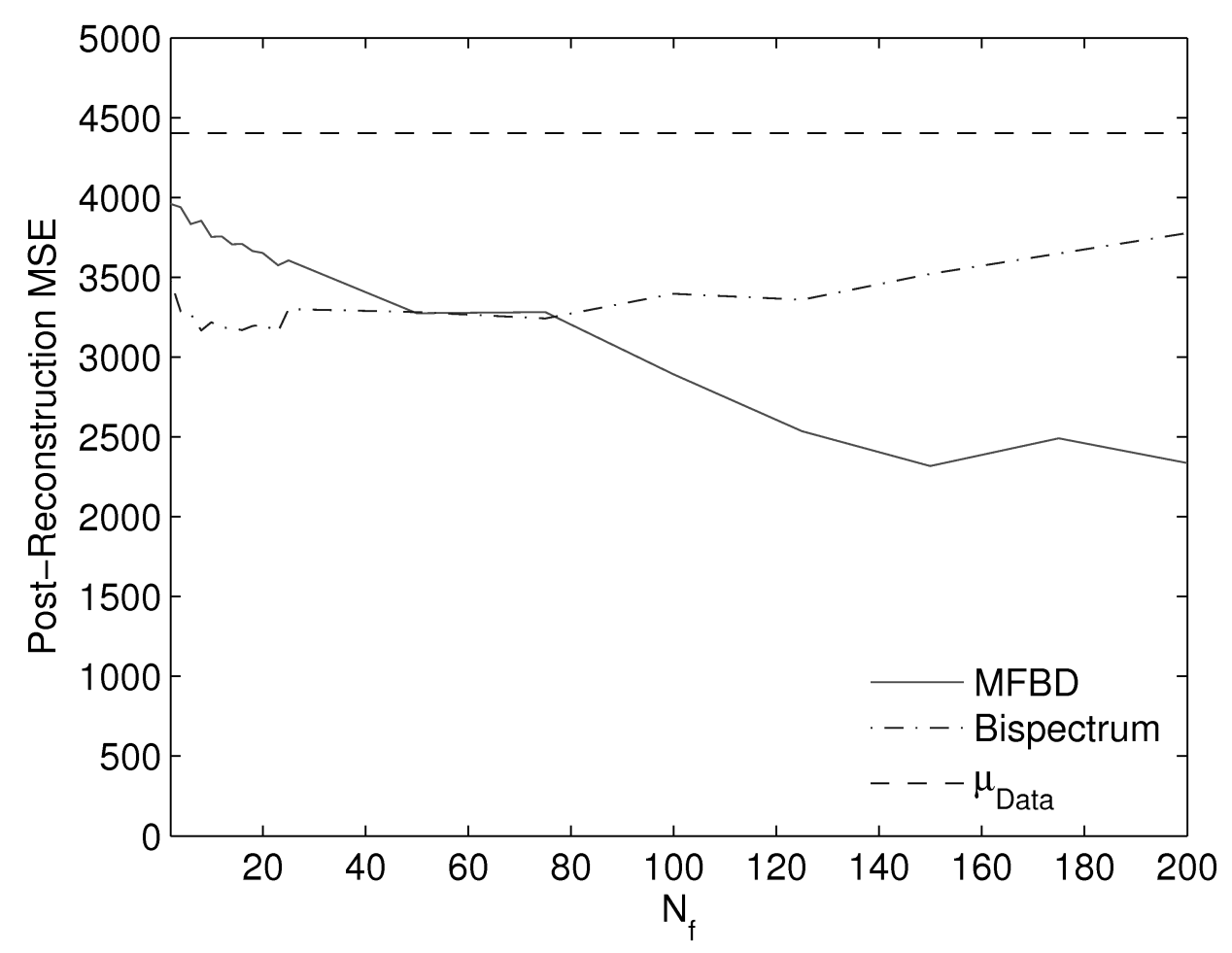

Figure 3.23: Comparison of Bispectrum and MFBD Mean Square Error as the number of input image frames is increased. For MFBD, $N_{z}=30$, and the number of iterations was fixed at 25. For Bispectrum, $N_{p}=5 \alpha=.3$. Poisson Case 2 Moderate turbulence $C_{N}^{2}=3.75 \times 10^{-14} m^{(-2 / 3)}$, mean photon rate $5 \times 10^{5} 8$ photons per pixel

\subsection{Conclusions}

The performances of a speckle imaging estimator and an unconstrained optimization-based multi-frame blind deconvolution estimator were compared in terms of the mean squared error between the object reconstructed using the method and a diffraction limited image. Three 1000-image data sets of a single image distorted by low, moderate, and severe turbulence that includes anisoplanatic effects were applied to both methods and their MSE 


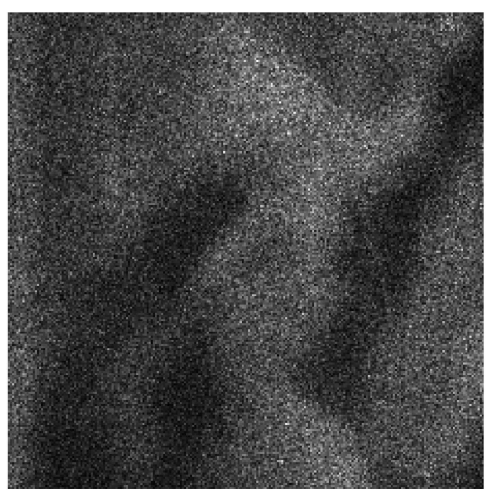

(a)

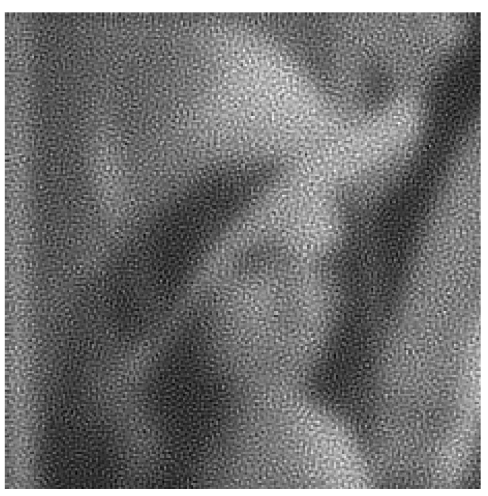

(b)

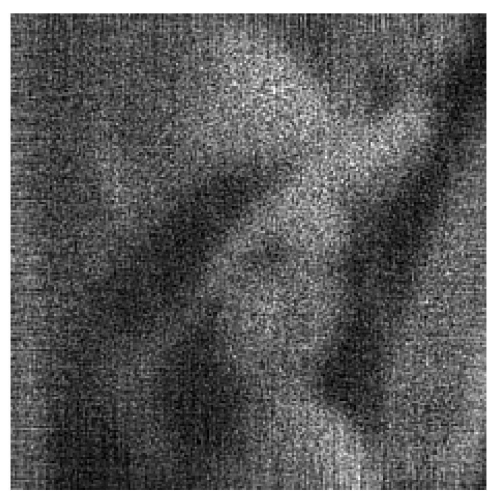

(c)

Figure 3.24: Case 3 Severe turbulence $C_{N}^{2}=5.25 \times 10^{-14} m^{(-2 / 3)}$, mean photon rate $5 \times 10^{5}$.Compares (a) a sample turbulent image (b) a sample MFBD reconstructed object and (c) a sample bispectrum reconstructed object.

performance evaluated. At normal illumination levels a wide range of turbulence cases would be well served by either SI or MFBD. Point performance estimates, using a data set of 1000 simulated turbulence corrupted images, indicate that speckle-imaging techniques reduce the MSE 46, 42 and 47 per cent on average for low, moderate, and severe turbulence conditions respectively using 15 input frames under daytime conditions and moderate frame rates. Similarly, the MFBD method provides, 40, 29, and 36 improvements in MSE on average under the same conditions. But the speckle techniques are significantly faster. The comparison is repeated under decreasing illumination conditions (less than 100 photons per pixel) where improvements of 39, 29 and 27 are available using speckle imaging methods and 25 input frames and 38, 34 and 33 respectively for the MFBD method and 150 input frames respectively under the assumption that the phase errors can be characterized as a Gaussian distribution. For all simulated turbulence cases, significant reductions were observed with as few as two input images. For the Poisson case, significant 


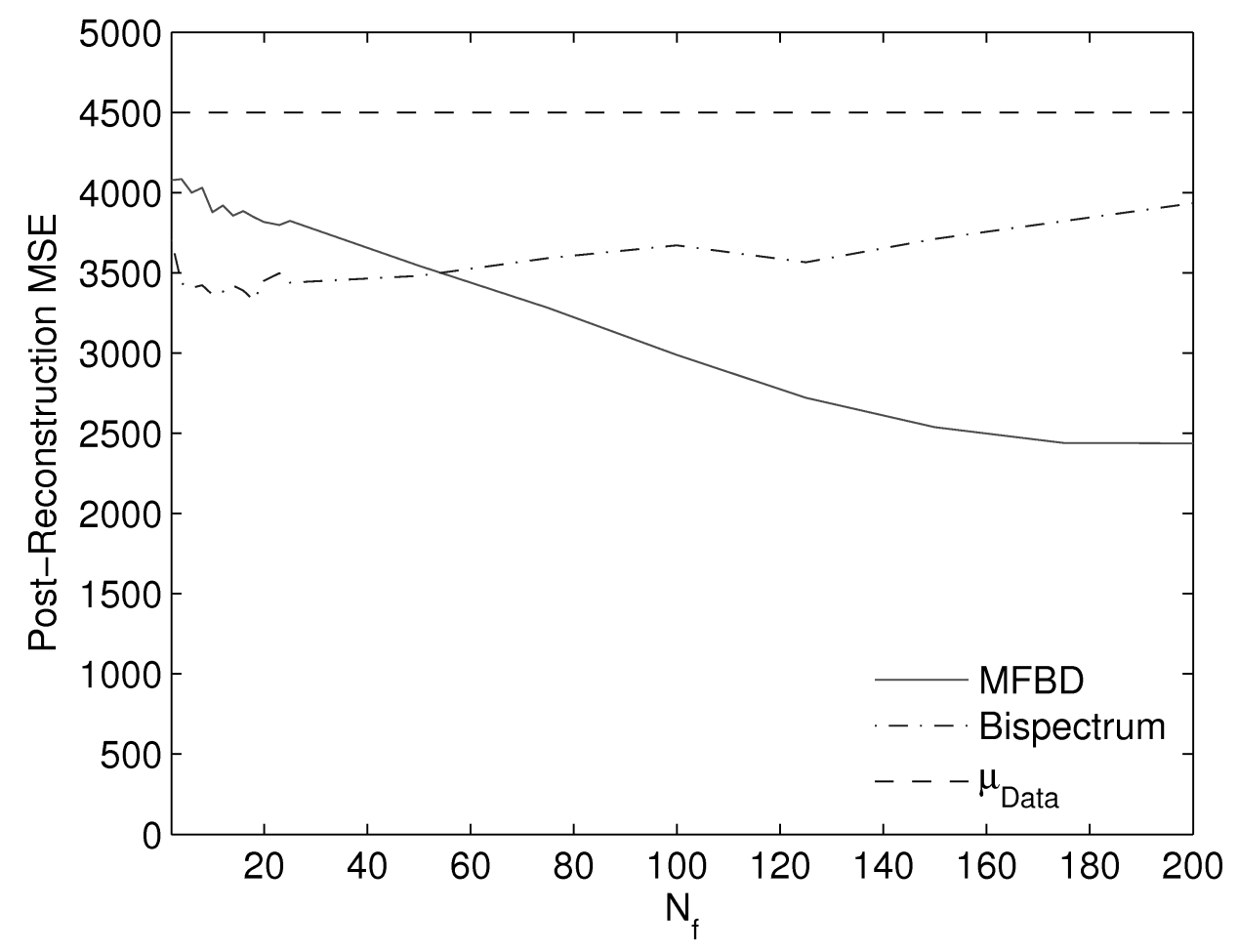

Figure 3.25: Bispectrum and MFBD Mean Square Error vs. number of frames. Poisson Case 3 Severe turbulence $C_{N}^{2}=5.25 \times 10^{-14}$, mean photon rate $5 \times 10^{5}$

results were achieved with as few as 50 frames for MFBD and 2 frames for speckle. 


\section{Chapter 4}

\section{MFBD Estimator Performance With}

\section{Field Data}

\subsection{Introduction}

1 In this chapter I describe the performance of the MFBD estimator using field images that were gathered as an ancillary part of a free-space-laser communications experiment [35]. The purpose of this was to demonstrate the performance on real field data. The transmitter side of the experiment consisted of an $808 \mathrm{~nm}$ laser transmitter and a pinwheel target. The receiver end of the experiment was a $30 \mathrm{~cm}$ Celestron telescope located 3.046

${ }^{1}$ The material in this chapter has been accepted for publication in Optical Engineering. 
Table 4.1

Date, time, and wave front sensor turbulence measurement range taken at approximately the same time as the images in data set 1,2 , and 3 were

recorded

\begin{tabular}{|r|c|c|c|}
\hline \multicolumn{4}{|c|}{ Field Data Turbulence Parameters } \\
\hline \hline Data Set & Date & Time & $r_{0}$ (cm) \\
\hline Set 1 & 1 July 2009 & 14:00 EDT & 4.6 to 5.2 \\
\hline Set 2 & 14 July 2009 & 14:00 EDT & 2.3 to 2.4 \\
\hline Set 3 & 24 July 2009 & 19:00 EDT & 1.78 to 2.8 \\
\hline
\end{tabular}

$\mathrm{km}$ downrange. The imaging path extended horizontally over both land and water at approximately 250 meters above sea level. As part of the communications experiment simultaneous images and turbulence measurements were taken using a Shack-Hartmann wave front sensor and a Point Grey CCD camera. Morning and afternoon data recording was challenging because the image path was oriented with the telescope looking from East to West and the low azimuth sun saturated the camera. The images used in MFBD reconstruction were recorded in the absence of continuous WFS measurements because the active laser present in the images recorded for the communications experiment regularly saturated the image sensor. Turbulence measurements were taken for three 27 second intervals prior to and post image collection. WFS sensor data was not concurrent with the images. My assumption is that the atmospheric turbulence did not change more rapidly than the data could be recorded. The Fried parameter estimates for each data set, $r_{0}$ are shown in Table 4.1 


\subsection{EDT 1 July, 14 July 2009 Image Processing}

Images from the 1 July and 14 July 2009 data set were processed using the MFBD estimator. A $250 \times 250$ pixel region of interest was extracted from the recorded images and padded up to $256 \times 256$ by replicating the pixels at the margin of the image outward. 15 images were processed using a spatially averaged initial estimate. The images were applied to the estimator for 20 iterations and the result is shown in Figures 4.1 and 4.2

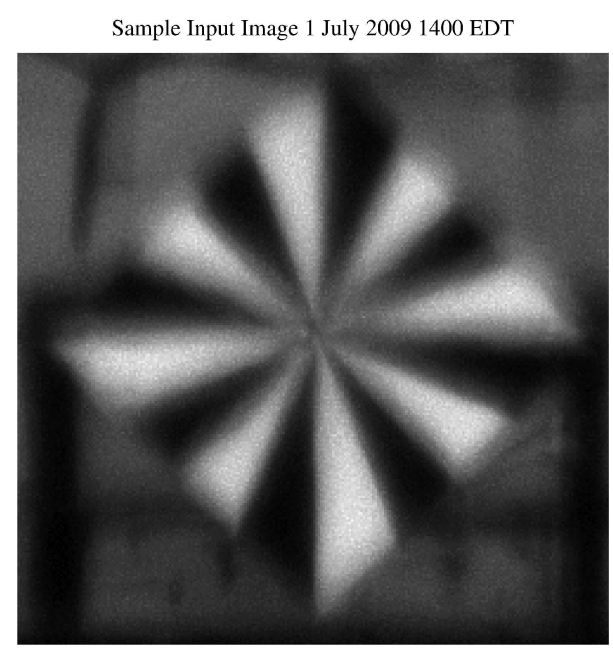

(a)

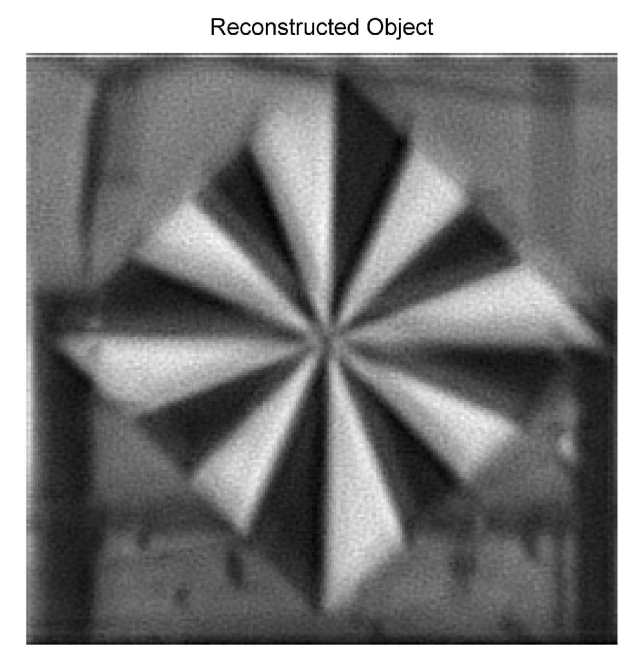

(b)

Figure 4.1: Field Data Reconstruction from 1 July 1400 EDT data set, using the Gaussian noise model MFBD reconstructor, $N_{f}=15, N_{\text {iter }}=20, r_{0}$ $4.6-5.2 \mathrm{~cm}$. A sample input image is shown in Figure (a) and a sample reconstructed image in Figure (b). 
Sample Image 14 July 20091400 EDT

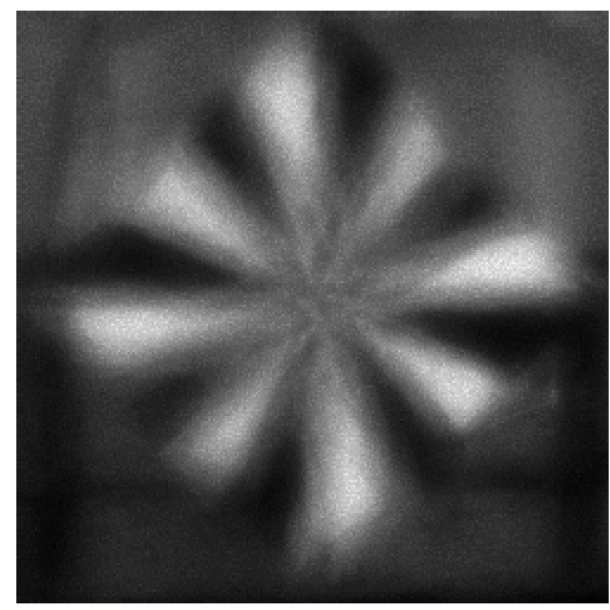

(a)
Reconstructed Object, $\mathrm{N}_{\mathrm{f}}=20, \mathrm{~N}_{\mathrm{z}}=35$, Iterations $=20$

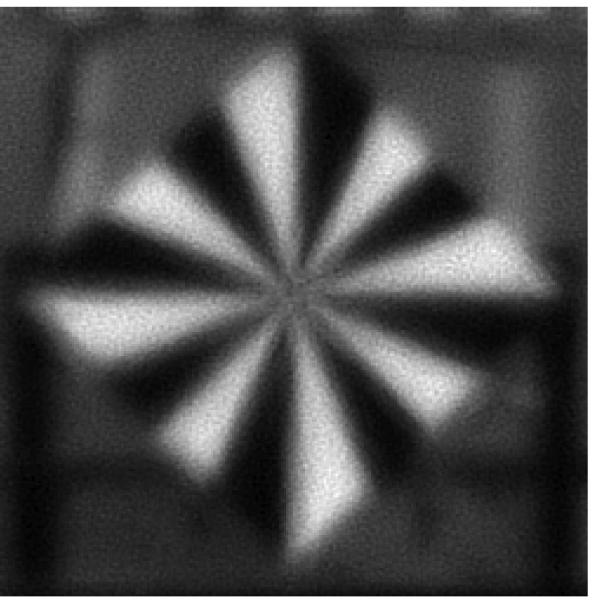

(b)

Figure 4.2: Field Data Reconstruction from 14 July 1400 EDT data set, using the Gaussian noise model MFBD reconstructor, $N_{f}=15, N_{\text {iter }}=20$, $r_{0} 2.3-2.4 \mathrm{~cm}$. A sample input image is shown in Figure (a) and a sample reconstructed image in Figure (b)

\subsection{EDT 24 July 2009 Image Processing}

Images from the 24 July 20091900 EDT data set were processed using the MFBD estimator. A 250 X 250 pixel region of interest was extracted from the recorded images and padded up to $256 \mathrm{X} 256$ by replicating the pixels at the margin of the image outward. $R_{0}$ for this data set ranged from $1.78-2.8 \mathrm{~cm}$, substantially smaller than data set 1 . To provide more information for the estimator to work with, 25 images were processed using 35 Zernike coefficients and 20 iterations of the estimator. A spatially averaged image calculated from the current image stack, was used as an initial estimate. The images were applied to the estimator and the result is shown in Figure 4.3 


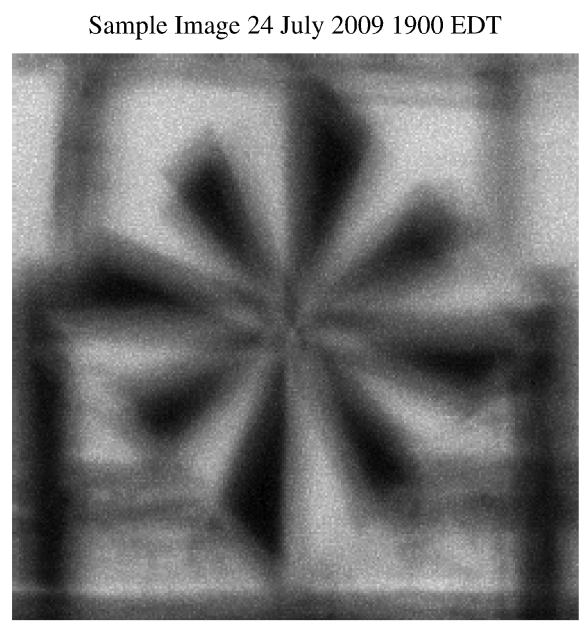

(a)
Reconstructed Object, $\mathrm{N}_{\mathrm{f}}=20, \mathrm{~N}_{\mathrm{z}}=35$, Iterations $=20$

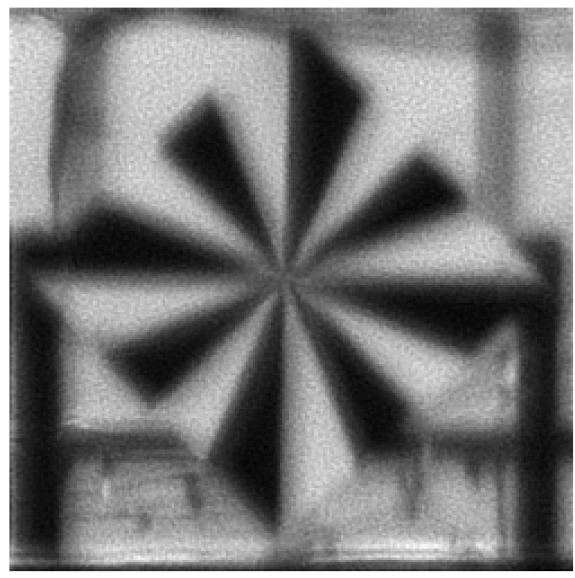

(b)

Figure 4.3: Field Data Reconstruction from 24 July 1900 data set, using the Gaussian noise model MFBD reconstructor, $N_{z}=35, N_{f}=25, N_{\text {iter }}=20, r_{0}$ $1.78-2.8 \mathrm{~cm}$. A sample input image is shown in Figure (a) and a sample reconstructed image in Figure (b)

\subsection{Conclusions}

Processing with 1July 2009 and 14 July 2009 field data with the MFBD reconstruction produced results qualitatively similar to those achieved with the simulated anisoplanatic images reported in Chapter 2 and 3, over a modest range of atmospheric turbulence. In the presence of stronger turbulence, evidenced by the smaller estimated values of $R_{0}$ for the 24 July data set, the MFBD processing required additional input frames to be applied to the estimator to achieve an output that was qualitatively similar to the 1July and 14 July data sets. This points to the need for some options for a field system that would enable the operator to adjust the number of images, the exit criteria for the estimator and the number 
of Zernike coefficients that result in a reconstruction that provides the "best" image for the user. 


\section{Chapter 5}

\section{The Effect of Using Bispectrum as the}

\section{Initial Estimate for the MFBD Estimator}

\subsection{Introduction}

Both bispectrum and MFBD have strengths to recommend them. Bispectrum produces rapid results but requires an estimate of $C_{n}^{2}$ and $\alpha$ to get started. The MFBD method converges more slowly but has the potential to produce a higher quality image ${ }^{1}$ The material in this chapter has been accepted for publication in Optical Engineering. 
reconstruction. However, MFBD requires an initial estimate of the object to get started and is subject to trapping in a local minimum of the cost function. The reduced run time of the bispectrum estimator suggests that it may offer a quick way to provide a better starting point for the MFBD estimator. Reconstruction processing in Chapters 2, 3 and 4 used the spatial average of the input images as a starting point for the L-BFGS optimization routine. In Chapter [ I describe an attempted hybrid strategy to combine the best of both techniques.

\subsection{Bispectrum initial estimate, limited iterations}

In the first case, a bispectrum estimate for the object intensities was used to replace the ensemble average of the input images as the starting point for the MFBD estimator and the number of iterations was used as the exit criteria for the estimator. The MSE was calculated as a function of the number of input frames used in the estimate. Setting the number of processing iterations at $N_{\text {iter }}=40$ and limiting the number of input frames to $N_{f}$ = 15 the hybrid method, B-MFBD, reduced the MSE by 16.9, 18.6, and 14.9 percent over MFBD processing using the ensemble average as the starting point when processing the low, moderate and severe turbulence data sets respectively[36]. When compared to the SI processing, reductions of 9.4, 8.4, and 6.6 percent in MSE were observed. Reconstruction times for each frame averaged approximately 1 minute for bispectrum, 10 minutes for MFBD, and 35 minutes for the B-MFBD hybrid. These results are summarized in Table

5.1. The hybrid B-MFBD improves on the performance of the standard MFBD estimator 
Table 5.1

Mean and variance for MFBD, Bispectrum and the hybrid B-MFBD methods for Low turbulence $C_{N}^{2}=2.25 \times 10^{-14} m^{(-2 / 3)}$, Moderate

Condition $C_{N}^{2}=3.75 \times 10^{-14} m^{(-2 / 3)}$, and Severe Condition $C_{N}^{2}=5.25 \times 10^{-14} m^{(-2 / 3)}$. Processing was accomplished with $N_{f}=15, N_{z}$ $=15$ and the exit criteria for the optimization routine set at 40 iterations.

\begin{tabular}{|c|c|c|c|c|c|c|}
\hline & \multicolumn{2}{|c|}{ MFBD } & \multicolumn{2}{c|}{ Bispectrum } & \multicolumn{2}{c|}{ B-MFBD } \\
\hline & $\mu$ & $\sigma$ & $\mu$ & $\sigma$ & $\mu$ & $\sigma$ \\
\hline Low & 431.0 & 32.9 & 395 & 21.0 & 358 & 32.9 \\
\hline Moderate & 617.0 & 94.5 & 548.5 & 28.5 & 502.6 & 32.9 \\
\hline Severe & 779.0 & 103.5 & 710.8 & 34.6 & 663.5 & 34.4 \\
\hline
\end{tabular}

and approximates the performance of the SI estimator. The significant characteristic of the B-MFBD estimator is an 4-6 percent improvement on the variance of the estimate over SI and MFBD applied by themselves as seen in Figures 5.1, 5.3, and 5.5.

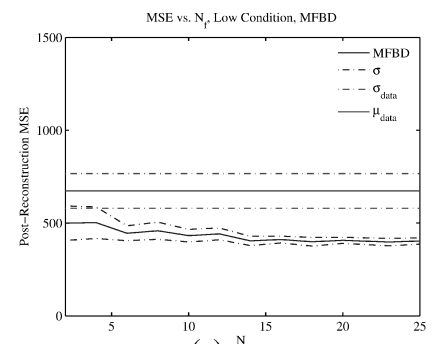

(a)

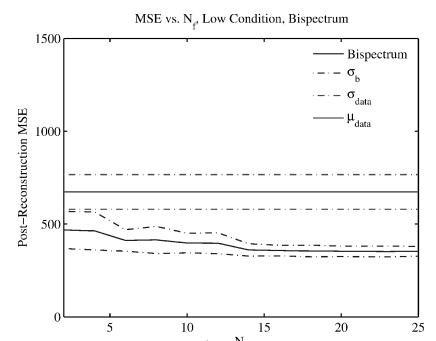

(b) ${ }^{N_{f}}$

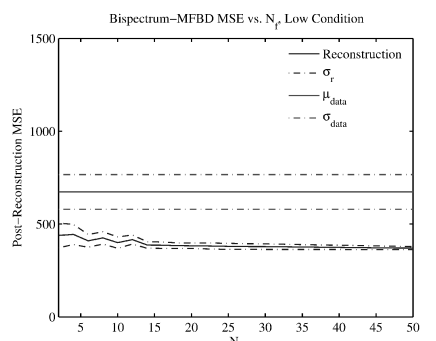

(c)

Figure 5.1: Mean Square Error performance vs. number of frames using the (a) MFBD alone, (b) Bispectrum alone, and (c) B-MFBD method using bispectrum to provide an initial estimate for processing with the MFBD estimator. Gaussian Case 1 low turbulence $C_{N}^{2}=2.25 \times 10^{-14} m^{(-2 / 3)}, N_{f}=$ 15. 


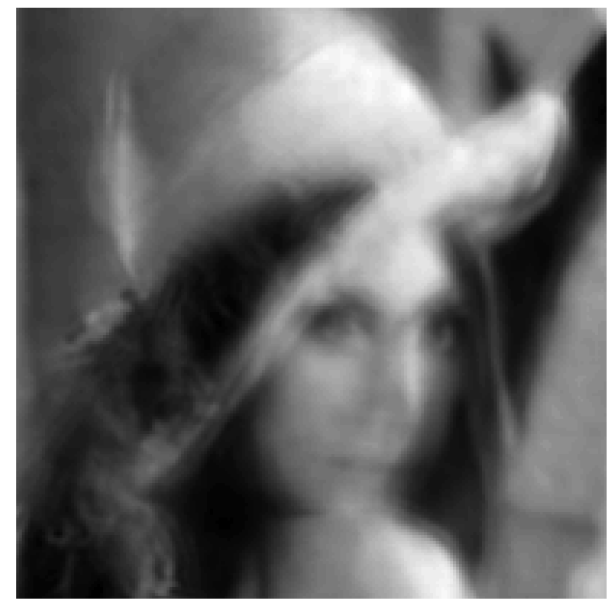

(a)

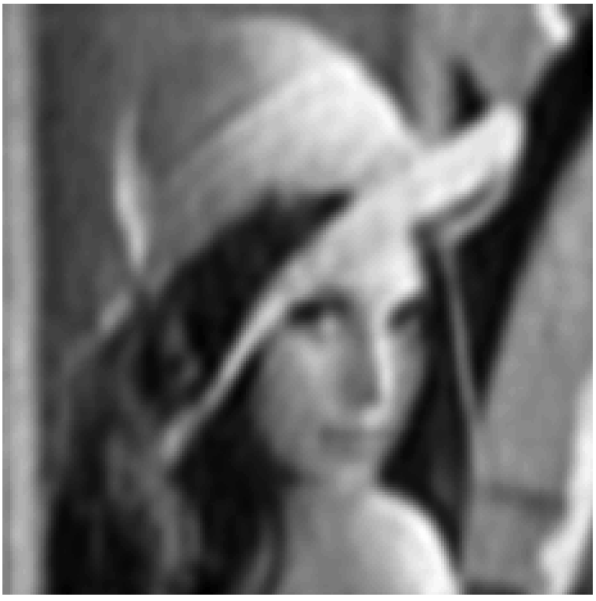

(b)

Figure 5.2: Limited iteration B-MFBD Samples, Gaussian Case 1 low turbulence $C_{N}^{2}=2.25 \times 10^{-14} \mathrm{~m}^{(-2 / 3)}$ images: (a) input frame, and (b) sample B-MFBD reconstructed object.

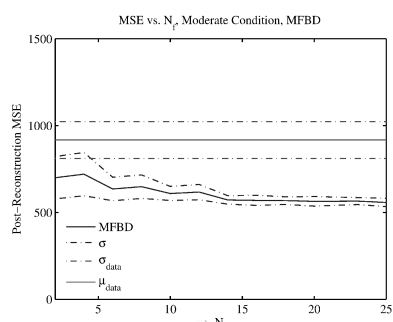

(a)

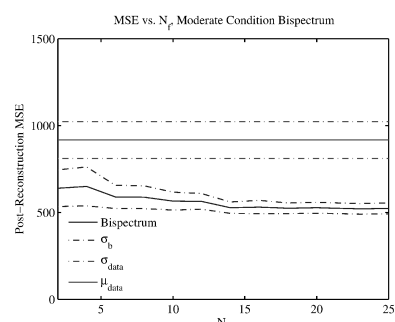

$(b)^{N}$

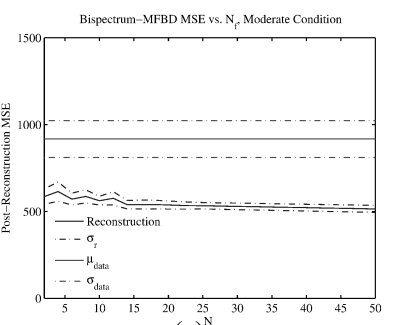

$(\mathrm{c})^{\mathrm{N}}$

Figure 5.3: Mean Square Error performance vs. number of frames using the (a) MFBD, (b) Bispectrum, and (c) B-MFBD method using bispectrum to provide an initial estimate for processing with the MFBD estimator. Gaussian Case 2 moderate turbulence $C_{N}^{2}=3.75 \times 10^{-14} m^{(-2 / 3)}, N_{f}=15$.

\subsection{B-MFBD run to convergence}

Further efforts to explore the performance characteristics of this technique used a value for the gradient of the cost function as the exit criteria for the L-BFGS optimization routine 


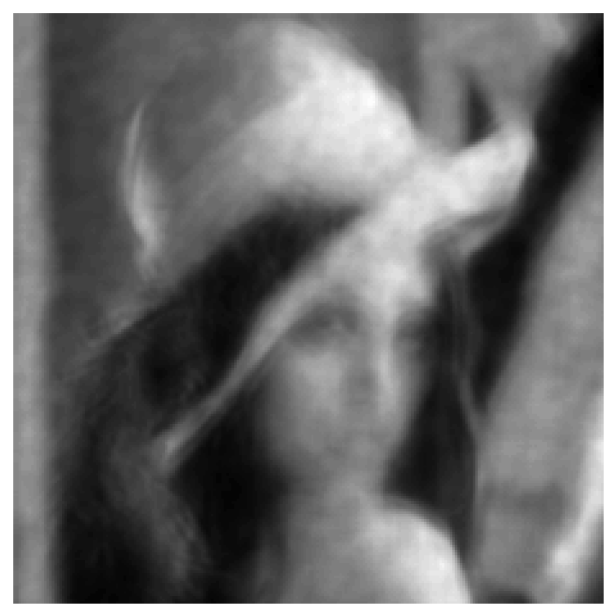

(a)

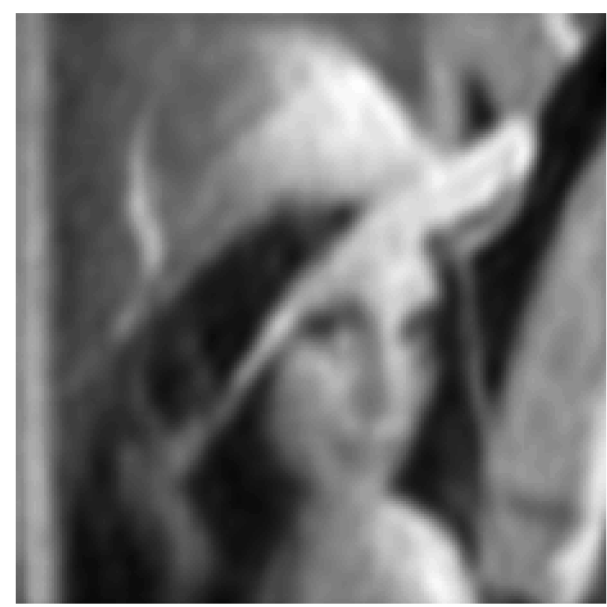

(b)

Figure 5.4: Limited iteration B-MFBD Samples, Gaussian Case 2 moderate turbulence $C_{N}^{2}=3.75 \times 10^{-14} m^{(-2 / 3)}:$ (a) input frame, and (b) sample B-MFBD reconstructed object.

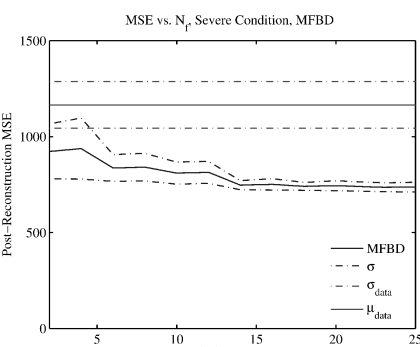

$\left(\right.$ a) ${ }^{\mathrm{N}_{\mathrm{f}}}$

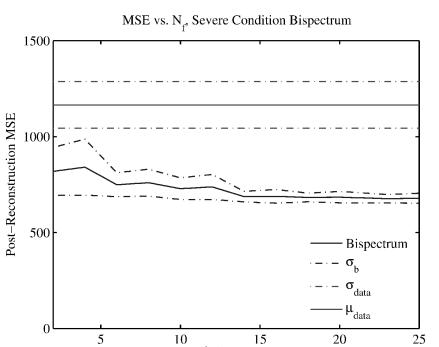

(b) ${ }^{N_{t}}$

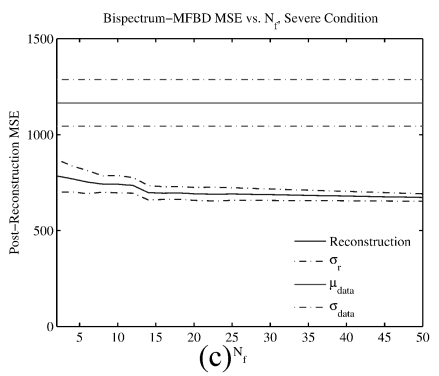

Figure 5.5: Mean Square Error performance vs. number of frames using the (a) MFBD, (b) Bispectrum, and (c) B-MFBD method using bispectrum to provide an initial estimate for processing with the MFBD estimator. Gaussian Case 3 severe turbulence $C_{N}^{2}=5.25 \times 10^{-14} m^{(-2 / 3)}, N_{f}=15$.

and allowed the estimator to run until it converged. A value of $\mathrm{g}=10,000$ was set and the B-MFBD estimator allowed to run until convergence produced the object estimates seen in Figures 5.7 (c), 5.8 (c), and 5.9 (c). Because processing times are both long and unpredictable, this technique is unlikely to see application in field system. 


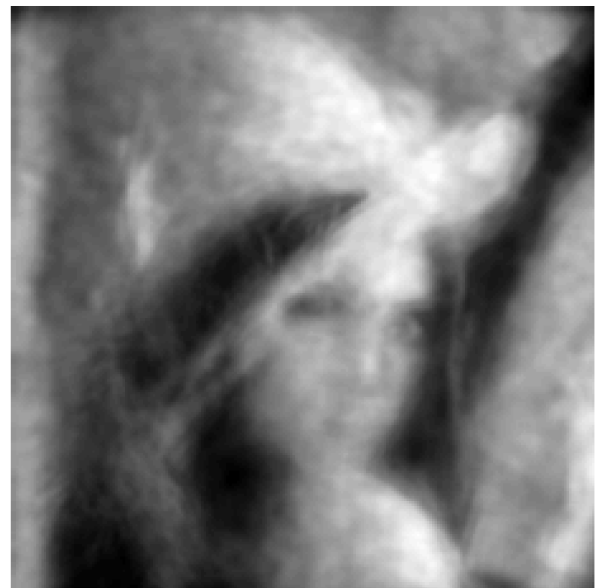

(a)

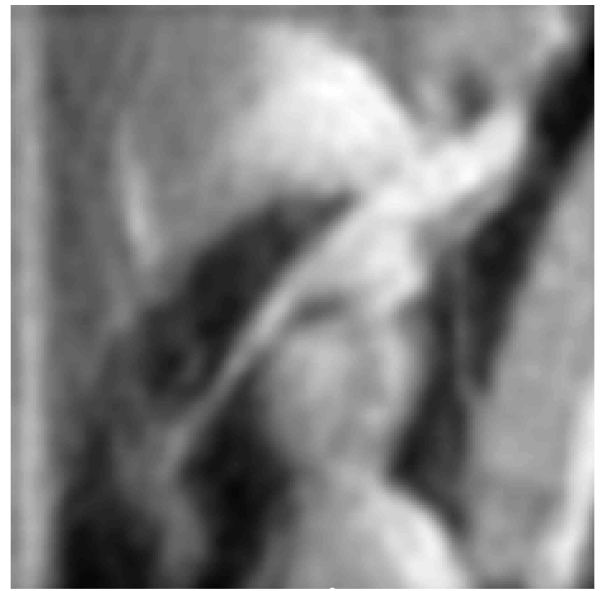

(b)

Figure 5.6: Limited iteration B-MFBD Samples, Gaussian Case 3 severe turbulence $C_{N}^{2}=5.25 \times 10^{-14} m^{(-2 / 3)}$ images: (a) input frame, (b) sample B-MFBD reconstructed object

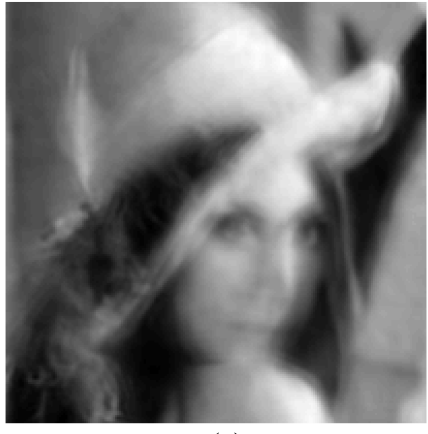

(a)

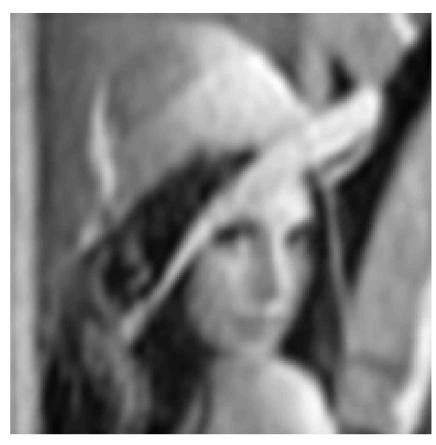

(b)

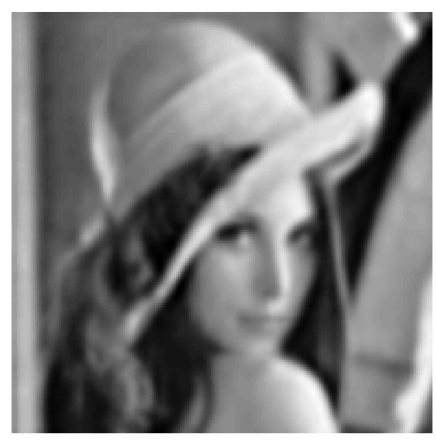

(c)

Figure 5.7: B-MFBD run to convergence using SI initial estimate. (a) Low turbulence condition input image sample, (b) Initial estimate from SI as MFBD starting point, (c) B-MFBD output when run to convergence

\subsection{Conclusions}

The B-MFBD estimator using the bispectrum estimator to provide the MFBD processing algorithm with an initial starting point produces modest MSE performance improvement 


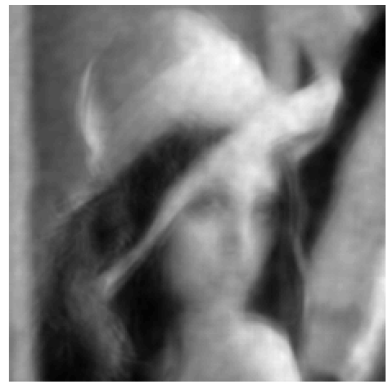

(a)

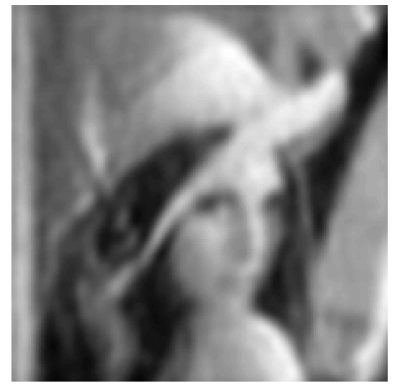

(b)

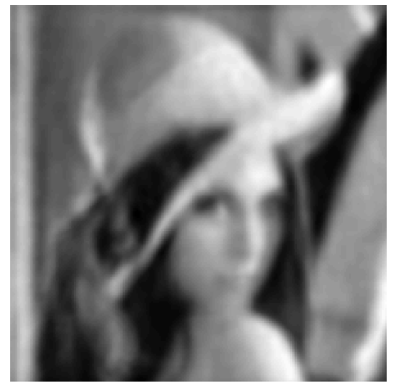

(c)

Figure 5.8: B-MFBD run to convergence using SI initial estimate. (a) Moderate turbulence condition input image sample, (b) Initial estimate from SI as MFBD starting point, (c) B-MFBD output when run to convergence

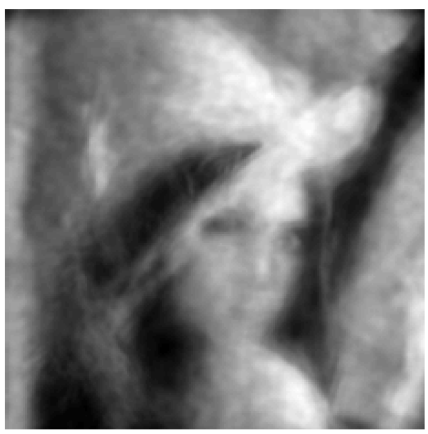

(a)

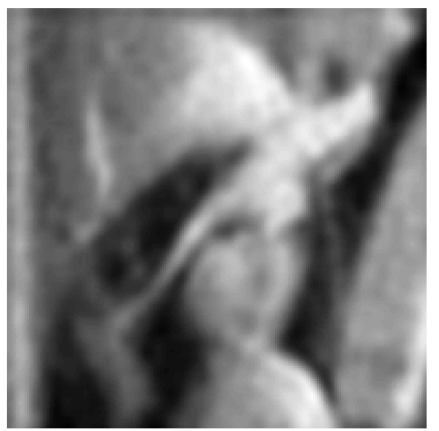

(b)

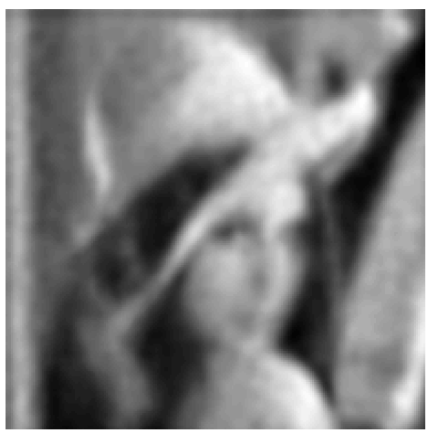

(c)

Figure 5.9: B-MFBD run to convergence using SI initial estimate. (a) Severe turbulence condition input image sample, (b) Initial estimate from SI as MFBD starting point, (c) B-MFBD output when run to convergence over speckle imaging and MFBD alone when 15 input frames are used in the reconstruction with 15 Zernike terms and 40 iterations of the MFBD algotithm. However, the hybrid B-MFBD method produced substantial improvement in the estimate variance. Qualitative improvements were achieved by letting the optimization run until convergence but this technique may not be suitable for embedded system implementation because of long, unpredictable processing times. 


\section{Chapter 6}

\section{Conclusion and Thoughts on Future}

\section{Work}

\subsection{Conclusion}

In Chapter 2 I found that it is possible to jointly estimate the pixel intensities and the Zernike coefficients using a multi-frame blind deconvolution estimator and that the MFBD reconstructed images showed significant improvement in mean squared error(MSE) compared to the average MSE for low, moderate, and severe atmospheric turbulence conditions using a generalized set of parameters selected in advance. This quality sets the stage for the development of a robust system that could be operated by unsophisticated 
users under a wide variety of seeing conditions. For the Gaussian noise model case the improvement in MSE was 40 percent for the low turbulence case using as few as 14 images and 30 Zernike coefficients in the reconstruction, 25 percent for moderate turbulence, and 36 percent for severe turbulence case. Parametric study of the number of Zernike terms useful in reconstruction showed that using more that 30 terms did not meaningfully improve the MSE. Image padding was assessed and I determined that padding the images with a combination of edge replicated pixels followed by zeros produced the best results. Processing times increased dramatically when the images were not padded to $N x N=2^{n} x 2^{n}$ to take advantage of MATLAB's FFT algorithms. The application of tapering filters at the margin between the image and the padding degraded the estimator's performance. The number of iterations allowed to the L-BFGS estimator, the number of frames used in the reconstruction and the number of Zernike polynomial coefficients used in the reconstruction had a significant, approximately linear, impact on the amount of time reconstructions required. In Chapter 3 I showed that at normal illumination levels a wide range of turbulence cases would be well served by either SI or MFBD. Point performance estimates, using a data set of 1000 simulated turbulence corrupted images, indicate that speckle-imaging techniques reduce the MSE 46, 42 and 47 per cent on average for low, moderate, and severe turbulence conditions respectively using 15 input frames under daytime conditions. Similarly, the MFBD method provides, 40, 29, and 36 improvements in MSE on average under the same conditions. But, the speckle techniques are significantly faster. When the comparison is repeated under decreasing illumination 
conditions (less than 30 photons per pixel, improvements of 39, 29 and 27 are available using speckle imaging methods and 25 input frames and 38, 34 and 33 respectively for the MFBD method and 150 input frames respectively under the assumption that the phase errors can be characterized as a Gaussian distribution. For all simulated turbulence cases, significant reductions were observed with as few as two input images. For the Poisson case, significant results were achieved with as few as 50 frames for MFBD and 2 frames for speckle. In Chapter 4 I demonstrated that processing with field data using the MFBD technique produced qualitatively similar results over a modest range of atmospheric turbulence. In Chapter 5 I demonstrated a novel hybrid B-MFBD estimator using the SI estimator to provide the MFBD processing algorithm with an initial starting point. The B-MFBD algorithm produces modest MSE performance improvement over speckle imaging alone using 15 input frames, 15 Zernike terms and 40 iterations of the MFBD algotithm. However, the hybrid B-MFBD method produced substantial improvement in the estimate variance. Qualitative improvements in the reconstructed object were achieved by letting the B-MFBD optimization process run until convergence but this technique may not be suitable for embedded system implementation because of long, unpredictable processing times. 


\subsection{Future Work}

The first thing I intend to explore is the relationship between the point spread function that is recovered by MFBD processing of the Lenna data sets and the point spread functions associated with pin-cushion images that are propagated along identicaly turbulent paths. This work is currently in progress.

Local minimum trapping is one of the hazards of the BFGS optimization algorithm. One strategy that can be employed to mitigate this problem is the use of simulated annealing. This could be applied in several ways. At the point where the initial guess at the object is made, whether by spatial averaging or the use of a bispectrum estimate, it is possible to perturb the guess slightly through the addition of a zero-mean, unity-variance random value to the object estimate. This result could be compared to the speed and MSE achieved when the initial guess Zernike coefficients are determined through multiple random draws which could be quickly evaluated against the cost function prior to application of the L-BFGS algorithm. A better initial guess should produce a better reconstruction, more quickly.

The MFBD optimization algorithm is one of many ways to maximize the log-likelihood functions developed in Chapters 2 and 3 . The use of genetic algorithms to achieve that goal may offer opportunities to explore. In general genetic algorithms cope well with large numbers of variables, search over wide areas of the surface of complex cost functions and 
work well with parallel computing. Applying this technique to the reconstruction of images corrupted by atmospheric turbulence may prove fruitful. 


\section{References}

[1] J. P. Bos and M. C. Roggemann, "Mean squared error performance of speckle-imaging using the bispectrum in horizontal imaging applications," vol. 51, no. 11, p. 805603, 2011.

[2] S. Baiotti, G. Scazzola, G. Battaini, and E. Crovari, Advances in UAV Data Links: Analysis of Requirement Evolution and Implications on Future Equipment. Ft Belvoir, VA: Defense Technical Information Center, 2000.

[3] A. Labeyrie, "Attainment of diffraction limited resolution in large telescopes by fourier analysing speckle patterns in star images," Astronomy and Astrophysics, vol. 6, p. 85 , May 1970.

[4] G. R. Ayers and J. C. Dainty, "Iterative blind deconvolution method and its applications," Opt. Lett., vol. 13, pp. 547-549, Jul 1988.

[5] A. W. Lohmann, G. Weigelt, and B. Wirnitzer, "Speckle masking in astronomy: triple correlation theory and applications," Appl. Opt., vol. 22, no. 24, pp. 4028-4037, 1983. 
[6] G. R. Ayers, M. J. Northcott, and J. C. Dainty, "Knox-thompson and triple-correlation imaging through atmospheric turbulence," J. Opt. Soc. Am. A, vol. 5, no. 7, pp. 963-985, 1988.

[7] R. J. Noll, "Zernike polynomials and atmospheric turbulence," J. Opt. Soc. Am., vol. 66, pp. 207-211, Mar 1976.

[8] R. A. Gonsalves and R. Chidlaw, "Wavefront sensing by phase retrieval," in Society of Photo-Optical Instrumentation Engineers (SPIE) Conference Series (A. G. Tescher, ed.), vol. 207 of Society of Photo-Optical Instrumentation Engineers (SPIE) Conference Series, pp. 32-39, 1979.

[9] B. J. Thelen, R. G. Paxman, D. A. Carrara, and J. H. Seldin, "Maximum a posteriori estimation of fixed aberrations, dynamic aberrations, and the object from phase-diverse speckle data," J. Opt. Soc. Am. A, vol. 16, pp. 1016-1025, May 1999.

[10] B. J. Thelen, R. G. Paxman, D. A. Carrara, and J. H. Seldin, "Overcoming turbulence-induced space-variant blur by using phase-diverse speckle," J. Opt. Soc. Am. A, vol. 26, pp. 206-218, Jan 2009.

[11] R. G. Paxman, T. J. Schulz, and J. R. Fienup, "Joint estimation of object and aberrations by using phase diversity," J. Opt. Soc. Am. A, vol. 9, pp. 1072-1085, Jul 1992. 
[12] D. Fraser, G. Thorpe, and A. Lambert, "Atmospheric turbulence visualization with wide-area motion-blur restoration," J. Opt. Soc. Am. A, vol. 16, pp. 1751-1758, Jul 1999.

[13] T. J. Schulz, "Multiframe blind deconvolution of astronomical images," J. Opt. Soc. Am. A, vol. 10, pp. 1064-1073, May 1993.

[14] M. A. Vorontsov and G. W. Carhart, "Anisoplanatic imaging through turbulent media: image recovery by local information fusion from a set of short-exposure images," Journal of the Optical Society of America A, vol. 18, pp. 1312-1324, Jun 2001.

[15] C. J. Carrano, “Speckle imaging over horizontal paths,” vol. 4825, pp. 109-120, Proc. SPIE, 2002.

[16] P. F. Curt, M. R. Bodnar, F. E. Ortiz, C. J. Carrano, and E. J. Kelmelis, "Real-time embedded atmospheric compensation for long-range imaging using the average bispectrum speckle method," Proc. SPIE, vol. 7244, p. 12, 2009.

[17] A. N. Kolmogorov, "The local structure of turbulence in incompressible viscous fluid for very large reynolds numbers," Proceedings: Mathematical and Physical Sciences, vol. 434, no. 1890 , pp. pp. 9-13, 1991.

[18] M. C. Roggemann and B. M. Welsh, Imaging Through Turbulence. Boca Raton, FL: CRC Press, 1996. 
[19] J. C. Wyngaard, Y. Izumi, and J. Stuart A. Collins, "Behavior of the refractive-index-structure parameter near the ground," J. Opt. Soc. Am., vol. 61, no. 12 , pp. 1646-1650, 1971.

[20] R. E. Hufnagel, "Propagation through atmopspheric turbulence," in The Infrared Handbook (W. L. Wolfe and G. Zissis, eds.), Office of Naval Research, Department of the Navy, Arlington, VA, 1978.

[21] D. L. Fried, "Limiting resolution looking down through the atmosphere," J. Opt. Soc. Am., vol. 56, no. 10, pp. 1380-1384, 1966.

[22] D. L. Fried, "Anisoplanatism in adaptive optics," J. Opt. Soc. Am., vol. 72, no. 1, pp. 52-52, 1982.

[23] J. P. Bos and M. C. Roggemann, "Technique for simulating anisoplanatic image formation over long horizontal paths," Optical Engineering, vol. 51, no. 10, p. $101704,2012$.

[24] R. K. Tyson, "Conversion of zernike aberration coefficients to seidel and higher-order power-series aberration coefficients," Opt. Lett., vol. 7, pp. 262-264, Jun 1982.

[25] G. Conforti, “Zernike aberration coefficients from seidel and higher-order power-series coefficients," Opt. Lett., vol. 8, pp. 407-408, Jul 1983.

[26] M. Born and E. Wolf, Principles of Optics: Electromagnetic Theory of Propagation, Interference and Diffraction of Light (7th ed). Elmsford, NY: Pergamon Press, 1999. 
[27] G. E. Archer, J. P. Bos, and M. C. Roggemann, "Reconstruction of long horizontal-path images under anisoplanatic conditions using multiframe blind deconvolution," Optical Engineering, vol. 52, August 2013.

[28] J. W. Goodman, Introduction to Fourier Optics. Boston, MA: McGraw Hill, 1996.

[29] M. Wakin, "Lena512 standard test images."

[30] F. Harris, "On the use of windows for harmonic analysis with the discrete fourier transform," Proceedings of the IEEE, vol. 66, pp. 51 - 83, Jan 1978.

[31] The Mathworks Inc., "MATLAB." version 7.12.0.635 (R2011a), 2011.

[32] D. Leblanc, “L-BFGS.” MTRI, Ann Arbor MI, 2011.

[33] G. E. Archer, J. P. Bos, and M. C. Roggemann, "Mean squared error performance of mfbd nonlinear scene reconstruction using speckle imaging in horizontal imaging applications," vol. 8399 of Visual Information Processing XXI, p. 11, SPIE, SPIE Proceedings, 2012.

[34] M. R. Billings, P. A. and B. Stribling, "Mitigating turbulence-induced image blur using multi-frame blind deconvolution," in AMOS Technical Conference, (Wailea, HI), pp. 506-512, Sept. 2001.

[35] A. V. Sergeyev, M. C. Roggemann, and C. Demars, "Near the ground laser communication system: anisoplantic studies based on the PSF measurements," Proceeding of the SPIE, vol. 8038, p. 13, 2011. 
[36] J. P. Bos, G. E. Archer, and M. C. Roggemann, "Using speckle imaging techniques as a starting point for mfbd scene reconstruction from long horizontal-path, turbulence-degraded imagery," vol. 8874, p. 11, Oct 2013.

[37] Unknown, "Boats, USC-SIPI Image Database." 


\section{Appendix A}

\section{A.1 MFBD processing of the "Boats" Data Set}

In Chapter 2 I described the use of the MFBD processing with turbulent images based on the "Lenna" image and in Chapter 5 that algorithm was used to process field data which was submitted for publication in Optical Engineering. In addition to those data sets, three sets of date based on the "Boats" image [37] were processed. This image was processed using the propagation simulator described by Bos[23] using the same parameters: propagation distance, wavelength, aperture size, and $C_{n}^{2}$ values shown in Table 2.1 that were used on the "Lenna" data set used in Chapters 2 and 3 , The MSE performance of the MFBD reconstruction algorithm for the three turbulence cases, Gaussian Case 1 low turbulence $C_{N}^{2}=2.25 \times 10^{-14} m^{(-2 / 3)}$, Gaussian Case 2 moderate turbulence $C_{N}^{2}=$ $3.75 \times 10^{-14} m^{(-2 / 3)}$, and Gaussian Case 3 severe turbulence $C_{N}^{2}=5.25 \times 10^{-14} m^{(-2 / 3)}$ are similar to that demonstrated by MFBD processing with the "Lenna" image based data 
sets for similar turbulence levels. The results for all three data sets are shown in Figure

A.1. The diffraction limited image used to calculate the MSE performance of the estimator
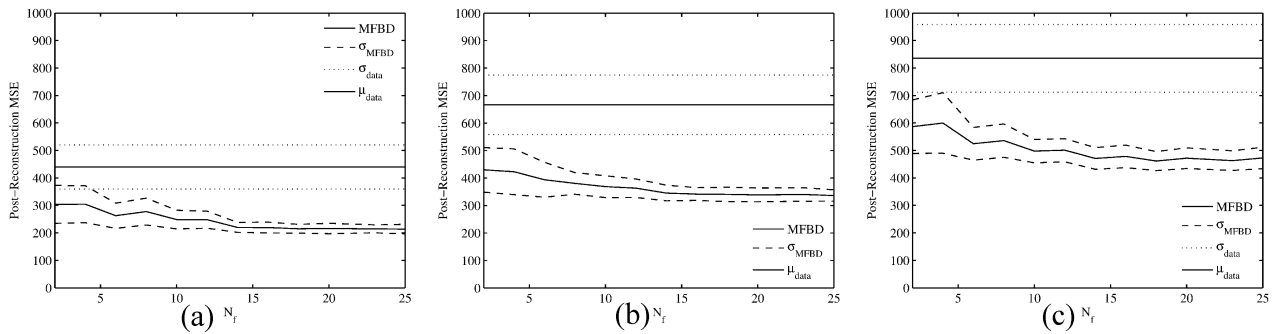

Figure A.1: Evaluation of Mean Square Error vs. number of frames used in the MFBD processing with $N_{z}=30$, and the number of iterations limited to 30. (a) Gaussian Case 1 low turbulence $C_{N}^{2}=2.25 \times 10^{-14} m^{(-2 / 3)}$, (b) Gaussian Case 2 moderate turbulence $C_{N}^{2}=3.75 \times 10^{-14} m^{(-2 / 3)}$, (c) Gaussian Case 3 severe turbulence $C_{N}^{2}=5.25 \times 10^{-14} m^{(-2 / 3)}$.

as described in Equation 2.25 is shown in Figure A.2 Samples of input image frames and reconstructions for Low, Moderate and Severe turbulence cases are shown in Figures A.3A.4 and A.5 


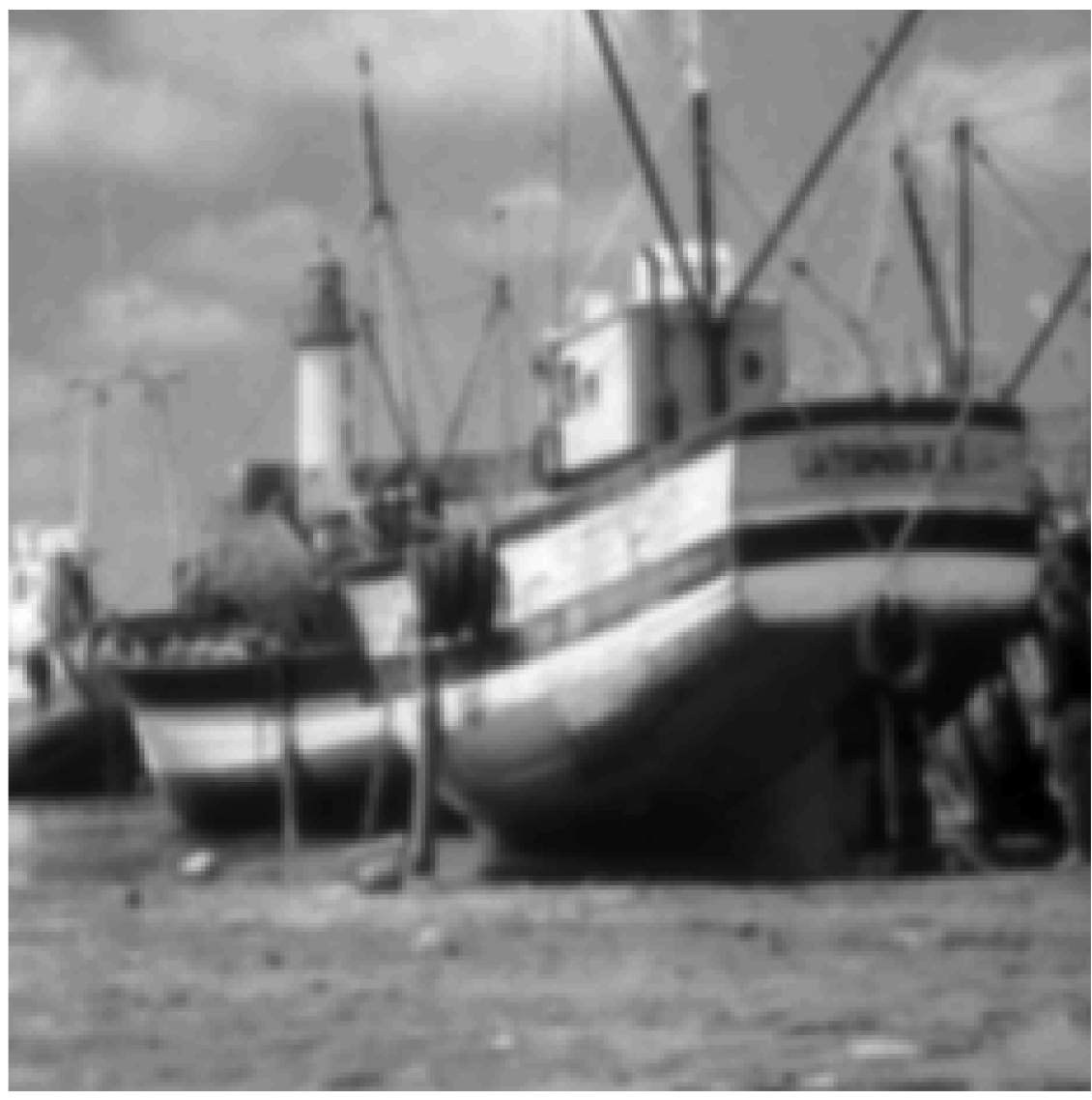

Figure A.2: Diffraction limited image from the "Boats" data sets.

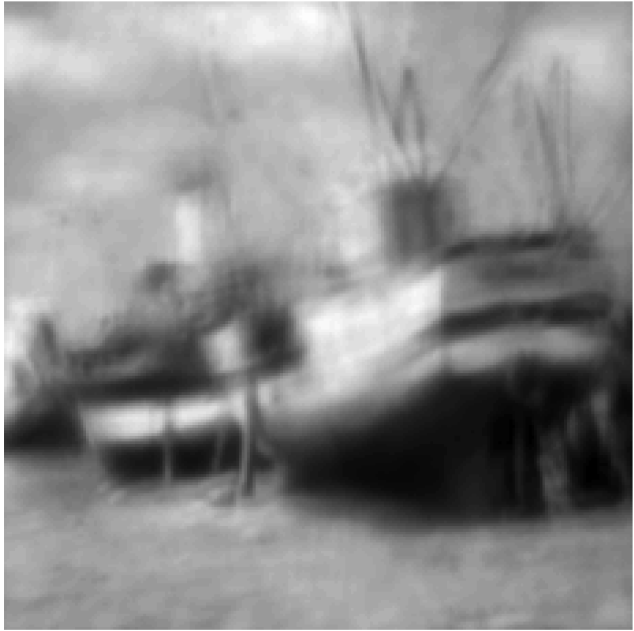

(a)

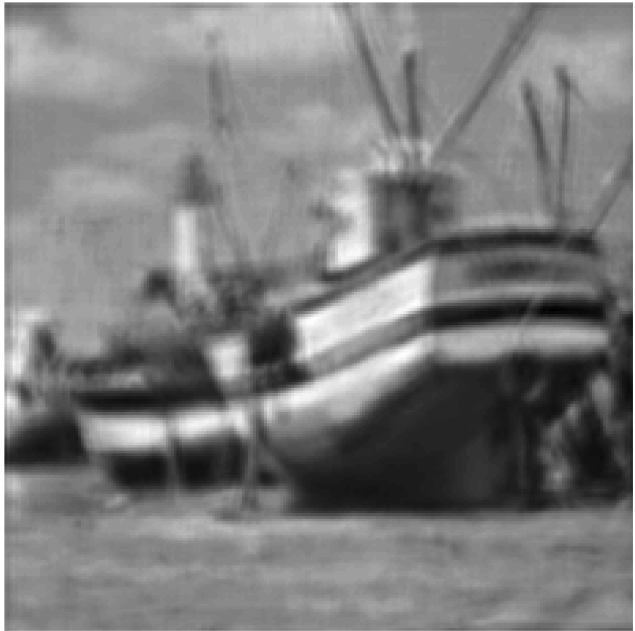

(b)

Figure A.3: Sample input and reconstructed image from the "Boats" data set processed with the MFBD algorithm, with $N_{z}=30$, and the number of iterations limited to 30. Gaussian Case 1 low turbulence $C_{N}^{2}=2.25 \times$ $10^{-14} m^{(-2 / 3)}$. 


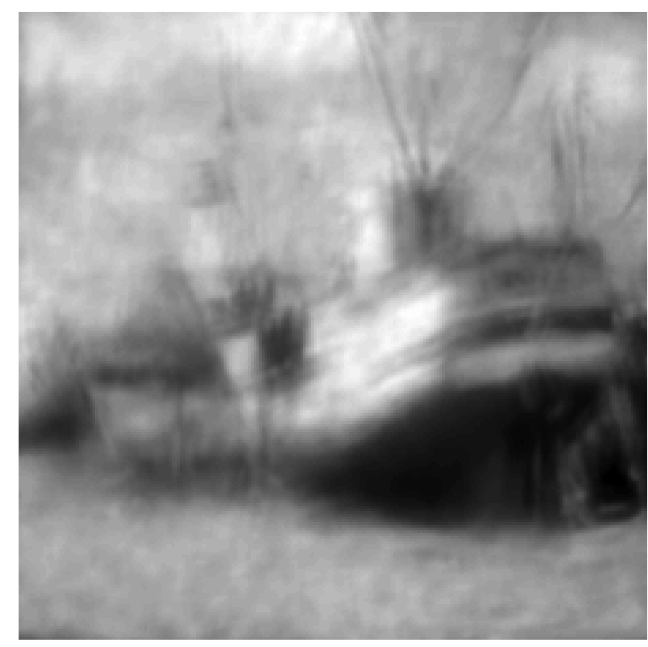

(a)

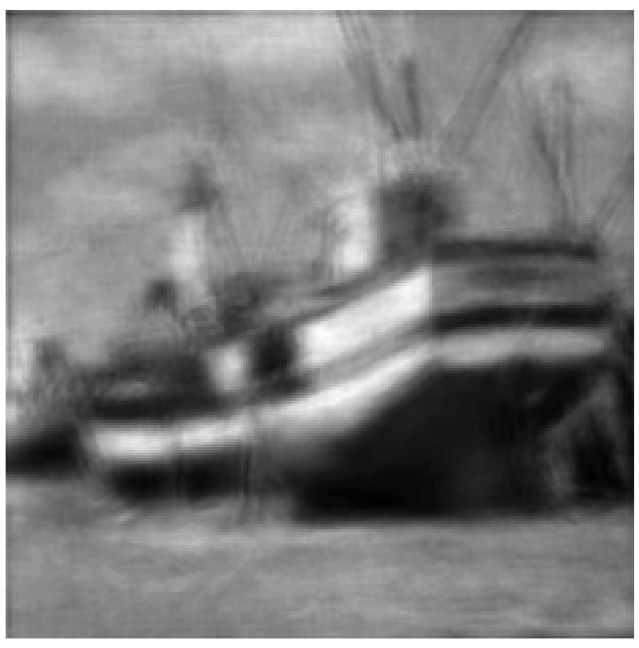

(b)

Figure A.4: Sample input and reconstructed image from the "Boats" data set processed with the MFBD algorithm, with $N_{z}=30$, and the number of iterations limited to 30 . Gaussian Case 2 moderate turbulence $C_{N}^{2}=3.75 \times$ $10^{-14} m^{(-2 / 3)}$.

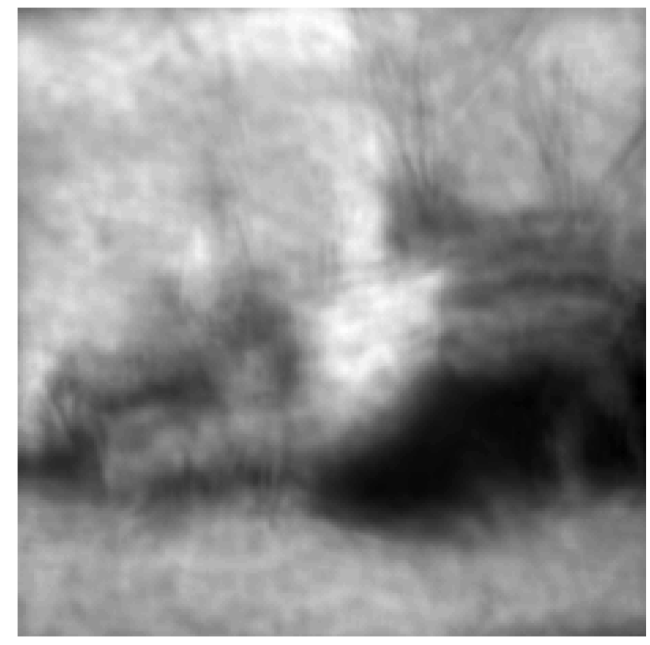

(a)

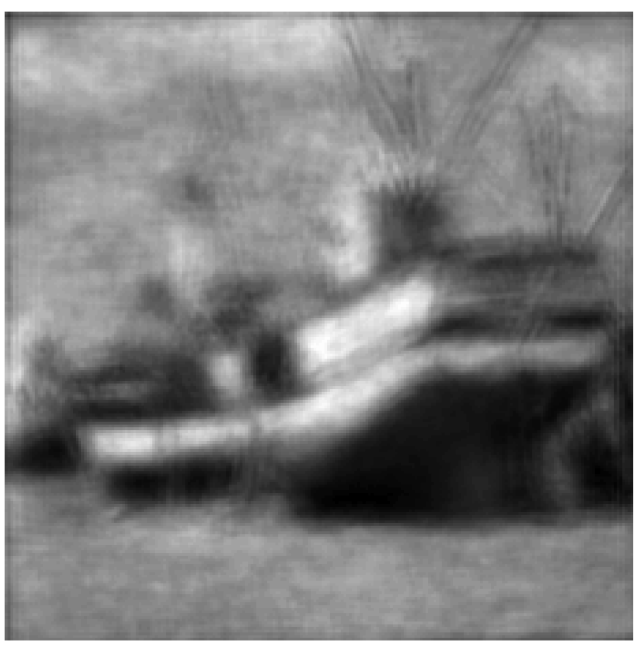

(b)

Figure A.5: Sample input and reconstructed image from the "Boats" data set processed with the MFBD algorithm, with $N_{z}=30$, and the number of iterations limited to 30, Gaussian Case 3 severe turbulence $C_{N}^{2}=5.25 \times$ $10^{-14} m^{(-2 / 3)}$. 


\section{Appendix B}

\section{B.1 Letters of Permission}

The content of Chapters 2, 3, 4, and 5 have been published or are under peer review for publication in the SPIE journal, "Optical Engineering." Optical Engineering's publication policy as stated in the SPIE Transfer of Copyright agreement, authors or their employers, in the case of works made for hire, retain the following rights: All proprietary rights other than copyright, including patent rights.

- The right to make and distribute copies of the Paper for internal purposes.

- The right to use the material for lecture or classroom purposes.

- The right to prepare deriviative publications based on the paper, including books or book chapters, journal papers, and magazine articles, provided that publication of a derivative work occurs subsequent to the official date of publication by SPIE. 
Thus, authors may reproduce figures and text in new publications. The SPIE source should be cited. 